\title{
Comparative clustering analysis of variable stars in the Hipparcos, OGLE Large Magellanic Cloud, and CoRoT exoplanet databases ${ }^{\star}$
}

\author{
L. M. Sarro ${ }^{1,2}$, J. Debosscher ${ }^{3}$, C. Aerts ${ }^{3,4}$, and M. López ${ }^{2}$
}

1 Dpt. de Inteligencia Artificial, UNED, Juan del Rosal 16, 28040 Madrid, Spain e-mail: 1sb@dia.uned.es

2 Spanish VO - LAEX-CAB (INTA-CSIC), Postal address. - LAEFF, European Space Astronomy Center (ESAC), PO Box 78, 28691 Villanueva de la Cañada, Madrid, Spain

3 Instituut voor Sterrenkunde, KU Leuven, Celestijnenlaan 200B, 3001 Leuven, Belgium

4 Department of Astrophysics, IMAPP, Radboud University Nijmegen, PO Box 9010, 6500 GL Nijmegen, The Netherlands

Received 9 March 2009 / Accepted 18 May 2009

\section{ABSTRACT}

\begin{abstract}
Context. Discovery of new variability classes in large surveys using multivariate statistics techniques such as clustering, relies heavily on the correct understanding of the distribution of known classes as point processes in parameter space.

Aims. Our objective is to analyze the correspondence between the classical stellar variability types and the clusters found in the distribution of light curve parameters and colour indices of stars in the CoRoT exoplanet sample. The final aim is to help in the identification on new types of variability by first identifying the well known variables in the CoRoT sample.

Methods. We apply unsupervised classification algorithms to identify clusters of variable stars from modes of the probability density distribution. We use reference variability databases (Hipparcos and OGLE) as a framework to calibrate the clustering methodology. Furthermore, we use the results from supervised classification methods to interpret the resulting clusters.

Results. We interpret the clusters in the Hipparcos and OGLE LMC databases in terms of large-amplitude radial pulsators in the classical instability strip and of various types of eclipsing binaries. The Hipparcos data also provide clear distributions for low-amplitude nonradial pulsators. We show that the preselection of targets for the CoRoT exoplanet programme results in a completely different probability density landscape than the OGLE data, the interpretation of which involves mainly classes of low-amplitude variability in main-sequence stars. Our findings will be incorporated to improve the supervised classification used in the CoRoT catalogue production, once the existence of new classes or subtypes will be confirmed from complementary spectroscopic observations.
\end{abstract}

Key words. methods: statistical - methods: data analysis - stars: binaries: eclipsing - stars: variables: general - stars: statistics techniques: photometric

\section{Introduction}

In the past decade, a plethora of new light curves of celestial objects has become available to the astronomical community. The OGLE project in its second stage (OGLE II), e.g., provided astronomers with a total number of $I$-band magnitude time series of the order of 40 million. Ongoing variability-focused projects such as ASAS (Pojmanski 2002), MOST (Matthews 2007) or CoRoT (Auvergne et al. 2009), together with other future survey databases such as those expected from the Panoramic Survey Telescope \& Rapid Response System (abbreviated as Pan-STARRS ${ }^{1}$ ), the Large Synoptic Survey Telescope $\left(\mathrm{LSST}^{2}\right)$ or $\mathrm{Gaia}^{3}$, will provide us with a unique oportunity to discover

\footnotetext{
* The CoRoT space mission was developed and is operated by the French space agency CNES, with participation of ESA's RSSD and Science Programmes, Austria, Belgium, Brazil, Germany, and Spain.

1 http://pan-starrs.ifa.hawaii.edu/public/

2 http://www. lsst.org/lsst

${ }^{3}$ http://www.rssd.esa.int/Gaia
}

new variability classes, either thanks to their unprecedented capability to detect weak periodic signals (the MOST and CoRoT cases) or to the large number of objects observed (Gaia, PanSTARRS or the LSST).

The discovery of new types of objects in large astronomical databases is facilitated through the application of statistical techniques. In particular, in the regime of very few cases in a potentially new class, we would be dealing with a problem of outlier detection, whereas a sufficient number of examples in the new class allows for the use of clustering techniques for class discovery. In both cases, a priori knowledge of the spatial distribution of the known classes is essential.

In the general case, a variability survey can be seen as a collection of celestial objects for which we have determined a series of parameters describing the variability in the time series. In this work we concentrate on periodic signals of variable stars as a starting point for understanding the variability diversity that can be found in any of the aforementioned surveys. Thus, typical attributes used to describe the time series are the significant detected frequencies, fourier decomposition characteristics (harmonic amplitudes, amplitude ratios, phase differences, etc.) 
and colour indices. An example of the list of attributes used for supervised classification purposes can be found in Sarro et al. (2009). Each variable star can then be represented by a point in a multidimensional space, the axes of which are the attributes mentioned above. We can view the database as a realization of a random point process and we can approximate its originating probability density function by means of parametric or nonparametric density estimation methods. In the outlier detection regime, exotic or unusual objects will thus be defined as occupying regions of the parameter space characterized by low probability densities. This further requires a deep understanding of the noise properties in the random process, like spurious frequency detections or the errors for low amplitude signals. In the clustering regime, it is hoped that a good clustering algorithm is capable of detecting the new class as a cluster.

A few clustering studies of variable celestial objects have been performed in the past. Eyer \& Blake (2005) studied ASAS variability data using Autoclass (Cheeseman et al. 1988). They manually separated two types of variables according to the regularity of the light curves. Some $45 \%$ of the total sample of 1731 stars was considered to have a sufficiently regular behaviour and was classified using Fourier decomposition coefficients. The remaining $55 \%$ was characterized by their period and second, third and fourth moments of their light curve distribution. In the first group, Autoclass identified nine clusters. These were linked with eclipsing binaries (three clusters, two of which are relatively pure and a third one mixed with ambiguous light curves and wrong periods), Cepheids (two clusters, one with clear candidates, the other with dubious cases), RR Lyrae pulsators (one cluster with only four stars), Small Amplitude Variables (a generic name for a mixture of low signal-to-noise variables), and a last group without interpretation. Judging by the plots in Eyer \& Blake (2005), the latter cluster is characterized by periods in the range 0.1 to 40 days and low $R_{21}$ values, where $R_{21}$ is defined as the ratio between the amplitude of the first harmonic and the amplitude of the frequency itself, for the dominant frequency. Moreover, the corresponding phase difference of these two Fourier terms, $\phi_{12}$, has random values which points to almost sinusoidal light curves.

Brett et al. (2004) applied Self-Organized Maps (hereafter SOMs) to study the clustering structure of ROTSE preclassified light curves. They also applied the algorithm to an artificial dataset of light curves for calibration purposes. They folded the time series with the period and binned the observations in a fixed number of phase bins. The training of the SOM was carried out on a set of light curves previously classified in one of the following categories: detached eclipsing binaries, contact eclipsing binaries, $\delta$ Scuti stars, RR Lyrae ab, RR Lyrae c, and Cepheids. The results indicate that, with this approach and dataset, SOMs are capable of separating detached binaries, contact binaries and RR Lyrae ab stars in three distinct categories, whereas RR Lyrae c, $\delta$ Scuti stars and Cepheids are mixed in one cluster. As the authors point out, the lack of information on the period is the main cause for this confusion of otherwise easily separable classes. The SOM approach has several major drawbacks. First of all, it does not incorporate information known to be relevant for the separation of clusters (e.g., the harmonics of detected frequencies). Also, the binning process renders the method inapplicable for databases where incomplete phase coverage is common (e.g., the Hipparcos and Gaia databases). Further, it is not robust against spurious frequency detections, a common problem with eclipsing binaries where identical eclipses result in a detected frequency which is twice the orbital one. The folded light curve will thus not resemble the eclipsing binaries prototypes. Finally, the results have been obtained with a dataset which is the result of intensive selection, i.e., only high signal-to-noise variables with well determined periods and clear class assignments were used in the training of the SOM.

In this work we characterize the distribution of variable objects in parameter space. Natural groups formed in several reference variability databases, such as the Hipparcos, the OGLE II Large Magellanic Cloud, and the CoRoT exoplanet programme are considered. Any clustering analysis is intrinsically limited by the sampling properties of the database to which it is applied. Thus, we can only hope to describe the realm of stellar variability to the limits of each experiment and the algorithms used to describe it, i.e., its limiting magnitude, sampling properties, sensitivity and the efficiency of the frequency detection algorithms. The capability of characterizing weak multiperiodic signals in faint stars in the Magellanic Cloud is limited, e.g., and clustering results in that regime cannot produce clean samples for this database. In our approach, we keep all variable stars in the database for which at least one statistically significant frequency was detected, regardless whether they have been previously classified in other works or not. Our sample thus is as realistic with respect to the original probability density function as the data permits it to be. The goal is to understand as deeply as possible this point distribution, in order to be prepared for class discovery in the presently considered and other forthcoming variability databases resulting from Gaia, Pan-STARRS or LSST.

\section{Elements of a clustering approach}

There are many theoretical approaches to clustering in the specialized statistics literature. In astronomy, the two most popular clustering techniques are Bayesian parametric modelling (e.g., the Autoclass implementation) and SOMs. There are several reviews describing unsupervised classification or clustering techniques (see e.g. Jain et al. 1999). A description of recent advances in the field is out of the scope of this article, but we believe that a brief discussion of some of the main issues involved in this kind of multivariate analysis is necessary for the subsequent understanding and interpretation of our results.

Clustering techniques can be put into several categories depending on the viewpoint used to characterize them. For example, they can be divided into parametric and nonparametric techniques according to the hypothesis underlying the method. Nonparametric techniques make no a priori assumption regarding the shape of the dataset constituent clusters. A good example of nonparametric techniques are density based clustering techniques that identify cluster centres as the modes in a kernel based estimate of the point density. Obvious drawbacks of this approach are its incapacity to detect low contrast overdensities in the vicinity of dominant clusters if they do not result in local maxima, and the limitations inherent to the bias-variance tradeoff dependence of the kernel widths. Other nonparametric techniques include hierarchical clustering and Nearest Neighbours related algorithms.

On the other hand, parametric techniques are developed under the hypothesis that the database is a sample from a probability density distribution that either is a functional form (model) of the parameters, or can be approximated with a number of such functions. The simplest (yet useful) model is to assume that the data are generated from a distribution that is a linear combination of multivariate normal distributions with unit covariance matrices. This results in spherical clusters and linear boundaries between clusters. This simple model has the mean of the 
distribution and the width of the multivariate normal as parameters, and a popular algorithm to find them is the ExpectationMaximization algorithm that maximizes the likelihood of the data. The model can be made more flexible and sophisticated by allowing for more complex covariance matrices. This translates into clusters of unequal major axis in arbitrary orientations (i.e., not necessarily aligned with the parameter space axis). This is the case of Autoclass, except for the fact that it goes beyond maximum likelihood estimation by introducing priors for the parameters, thus turning into a fully Bayesian inference.

One advantage of parametric models is that they permit the detection of clusters not associated with local maxima, such as those missed by density based methods. Unfortunately, the evident disadvantage is that, if the underlying assumption is incorrect or insufficient (i.e., if the clusters are not samples from the hypothesized parametric model), the resulting clustering is uninterpretable. This drawback is not present in non parametric clustering, where clusters of arbitrary shape can be detected. Obviously, it is always possible to recontruct the real shape of a cluster by adding more and more model components (multivariate Gaussians in the most popular choice), but then the possibility to detect new stellar populations is compromised because the clusters are no longer linked to differences in the point samples, but are created by the inability of the algorithm to fit the real distribution of points.

Two other elements, central to the problem of finding a good clustering description of a dataset, are the automatic determination of an optimal number of clusters, and that of feature selection. The former is closely related to the clustering evaluation measures, since choosing a number of clusters relies on the availability of an objective measure of clustering quality. In the framework of parametric clustering, the classical tools for model selection are readily available. In the Bayesian approach, the Bayes factor is commonly used, which incorporates a natural Occam's razor by penalizing solutions with excessive number of parameters (clusters). Obviously, one can find a perfect match to any density distribution by adding more and more components to our mixture model. This would be the equivalent to the concept of overfitting in supervised classification or regression problems. Other ways to assess clustering quality related to maximum likelihood estimators are the Bayesian Information Criterion (BIC) and Akaike Information Criterion (AIC), or the Median Split Silouhettes (MSS, Pollard \& Laan 2002) and the gap statistic (Tibshirani et al. 2000), which can also be applied to nonparametric clustering.

The other critical aspect of clustering experiments is the selection of an optimal attribute set, capable of incorporating the attributes where clustering occurs but, at the same time, discarding those irrelevant to treat the problem. Unfortunately, this field has not evolved as much in unsupervised as in supervised classification, and there is no commonly accepted technique to perform attribute selection. In the experiments described in Sect. 4, we selected the attributes manually based on the past experience in supervised classification.

One last aspect that has to be considered in the selection of an algorithm for the clustering analysis of variablity data is that of scalability. Both the time and space complexities of the algorithms, and the possibility of parallelizing the data processing has to be considered, specially if the algorithms have to be applied to large databases such as those expected in the next few years.

This summary is not intended to be exhaustive but rather brings an introduction to the key aspects of the problem of class discovery in variability databases. There is simply no best clustering methodology for all types of problems. We stress the importance of making both the assumptions underlying each method and the evaluation criteria explicit before a clustering experiment is designed.

\section{Adopted methodology}

In designing the experiments and methodology described below, we imposed several requirements. The solution proposed here is an attempt to fulfil all of the following requirements:

1. Interpretability. We consider that no class discovery is possible if the clusters obtained by a given method are not interpretable in terms of the experts domain knowledge. In this case, the domain is that of regular variable stars and this means that the clusters defined by the method have to recover the classical variability types reasonably well. This requirement obviously has to be related to the parameter space where clustering is performed: it can not be hoped to separate variability classes only differing in some spectroscopic signature (e.g., due to metallicity effects) if the parameter space is built only with the properties of a photometric time series.

2. The algorithms involved in the clustering process have to be scalable to the orders of magnitude typical of the era of astronomical surveys. Nowadays, large scale variability surveys like those expected to come from Gaia, Pan-STARRS or LSST, will be producing parameters for $\approx 10^{9}$ objects or more, and class discovery techniques have to be capable to deal with these enormous databases.

3. The methodology has to be model free or, at least, use the most flexible models available, in order to avoid that the clusters obtained are dictated by the model selected.

In the following, we describe the clustering methodology adopted in the performed experiments. We decided to use the so-called density based approach to clustering, whereby clusters are identified with maxima of the density distribution, and points are assigned to their closest maximum or mode. This density is never actually computed and points are assigned to modes (clusters) by using the Modal EM (Expectation Maximization, MEM) algorithm ( $\mathrm{Li}$ et al. 2007), which can be summarized as follows. Let $S=\left\{x_{1}, x_{2}, \ldots, x_{n}\right\}$ be the set of objects $x_{i} \in \mathcal{R}^{d}$ to be clustered. The $d$ components in each $x_{i}$ are the attributes or parameters used to characterize the variable objects (frequencies, amplitudes, phase differences, colour indices, etc.; see below). Let $f(x)=\sum_{k=1}^{K} f_{k}(x)$ be the density of points approximated by a Mixture Model with $K$ components. In our case, we used multivariate normal distributions $\mathcal{N}_{\mu, \Sigma}(x)$, with mean $\mu$ and covariance matrix $\Sigma$. Then, given a point $x$, the MEM algorithm consists in alternating the following two steps, starting with $r=0$ and $x^{(0)}=x$ :

1. Expectation: compute the posterior probability of each mixture component, $p_{k}=\frac{\pi_{k} f_{k}\left(x^{(r)}\right)}{f\left(x^{(r)}\right)}$.

2. Maximization: update $x^{(r+1)}=\operatorname{argmax}_{x} \sum_{k=1}^{K} p_{k} \log \left(f_{k}(x)\right)$.

When applied to one object in the database defined by $x_{i}$, the MEM algorithm converges to a local maximum of the density estimate. Clustering is then accomplished by grouping points that converged to the same mode (to within some tolerance). Our starting point is a kernel density estimate such that, if the database contains $n$ objects, $f(x)$ is defined by a mixture model with $n$ components, one associated to each object:

$f(x)=\sum_{k=1}^{n} \frac{1}{n} \mathcal{N}_{x_{k}, \Sigma_{k}}(x)$. 
Given that the implementation has to be capable of processing datasets with a number of instances of the order of several times $10^{4}$ (in these experiments) and larger, the implementation makes use of $k$-dimensional trees ( $k d$-trees hereafter; see e.g. Freidman et al. 1977; Bentley 1980).

It is evident that the MEM algorithm involves an approximation to the density which, in this case, is a kernel based approximation. Therefore, depending on the kernel bandwidth $\Sigma_{k}$ used in the estimate, we will have different sets of modes: less nodes and large clusters for a large kernel width, and many small clusters when the kernel bandwidth is small. At each scale we are analysing different aspects of the dataset but, as the kernel width is made smaller, the modes of the density distribution can be made to correspond to local maxima due to noise in the finite sampling of the point process. Therefore, the smallest scales one is ready to accept have to be judged on physical terms in order to establish the significance of the clusters obtained.

In our case, we carried out the analysis in a hierarchical manner, starting with large kernel bandwidths to define the large groupings in the data. Thereafter, we decreased the widths until we could no longer establish the meaningfulness of the largest clusters obtained. We also implemented adaptive kernel widths in order to avoid large variances in the clustering results in the low density regions. The kernel width adaptation takes into account the local density of points in order to increase the width in regions where the nearest neighbours are at large distances and thus, fixed kernel estimation would result in a local maximum of the density at each observed object.

Since these experiments were designed to serve as the basis for subsequent class discovery, we concentrated the description of the clusters in terms of known classical variables. Thus, the clustering decomposition (the decrease in the kernel bandwidths) stopped when we no longer could interpret the resulting clusters.

\section{Performed experiments}

In the following, we describe the experiments we performed with three databases: the Hipparcos variability archive (Perryman \& ESA 1997), the OGLE database of Large Magellanic Cloud variables (Zebrun et al. 2001), and the CoRoT database corresponding to the first four runs (IRa01, SRc01, LRc01, and LRa01; Debosscher et al. 2009). These databases are characterized by very different statistical properties. While the sampling is quite regular but drastically different in the OGLE and CoRoT databases, this is less so in the Hipparcos case. The number of cases in each sample is also very different: 43351 variabels were retrieved from the OGLE LMC database, 2419 variables from Hipparcos and 14642 from CoRoT (see below for selection criteria). Finally, colours are available for very large fractions of the OGLE and Hipparcos databases whereas the CoRoT sample is analysed only based on parameters derived from the time series.

The figures given above for the number of objects in each database are the result of selecting sources with some predefined criteria. For both the OGLE and Hipparcos databases of variable stars, we selected objects with available $V-I$ colour index. For the Hipparcos and CoRoT databases we also requested $p$-values in the frequency detection phase below $10^{-7}$ (see Debosscher et al. 2009, for a definition of the $p$-values).

The original time series in each database was processed in order to detect statistically significant frequencies. The procedure was described in detail in Debosscher et al. (2007), Sarro et al. (2009), and Debosscher et al. (2009) and is therefore omitted here. We only retain five relevant attributes in the analysis below, namely the first detected frequency (denoted as $v$ in the OGLE and Hipparcos cases and as $v_{1}$ in the CoRoT case and expressed in $\mathrm{d}^{-1}$ throughout the paper), the amplitude of the first component in the Fourier decomposition of this frequency $A_{11}$, the $R_{21}$ ratio which stands for the ratio of the amplitudes of the first two components in the Fourier decomposition of the first frequency, the phase difference $\phi_{12}$ between the second and first harmonic components in the Fourier decomposition and (in the OGLE and Hipparcos cases), the $V-I$ colour index. In the CoRoT case, given the quality of the time series, we used the amplitudes of the first four components in the Fourier decomposition of the first detected frequency, and the first two of the second detected frequency $v_{2}$. Further attributes can be added in order to look deeper into the composition of the clusters found. This includes parameters related to higher orders in the Fourier decomposition and additional undereddened colour indices if available, such as $J-H$, or $H-K$. The latter only make sense for selected clusters where multiperiodicity or infrared emission are attributes expected to result in clustering structures at smaller scales.

In the following, we present and describe clustering results obtained with the density based methodology described in Sect. 3. As explained above, clusterings with different levels of detail can be obtained with different kernel bandwidths $\Sigma_{k}$ (the covariance matrix used in the normal distributions placed at each point). Thus, starting with a large kernel bandwidth (resulting in few large clusters), it is possible to refine and subdivide the clusters obtained by using increasingly smaller bandwidths. The resulting structure resembles a tree (the dendrogram) where clusters (branches) subdivide into smaller clusters at smaller scales.

The covariance matrix $\Sigma_{k}$ can be chosen isotropic $\left(\Sigma_{k}=\right.$ $\sigma^{2} \cdot \mathbb{1}$ ) with $\sigma^{2}$ being the tuning parameter effectively controlling the kernel bandwidth), or, more generally, anisotropic. In this work we have made the diagonal elements proportional to the nearest neighbour distance. The proportionality is introduced by normalizing the covariance matrix such that the maximum along the diagonal is made equal to the tuning parameter $\sigma^{2}$.

The parameters selected for the clustering process and the level at which the dendrogram is cut are mostly dictated by the interpretability of the clusters and clarity of plots. While it is possible to present purer clusters (i.e., with more homogeneous components) it is usually at the expense of a larger number of clusters. It is important to remark that each dimension of the data was normalized in the range $[0,1]$ to avoid attributes with larger absolute values to dominate the kernel variance. Therefore, the input data to the clustering algorithm are contained inside a unit hypercube. This means that the quoted values of $\sigma$ (the kernel bandwidth) are relative to this unit length defining the range in each dimension. The values used for each experiment (Hipparcos, OGLE LMC and CoRoT) were 0.2, 0.15 and 0.2 respectively. While, as explained above, the availability of colour indices and class labels in the first two experiments guided us in the selection of the actual value of the kernel width, in the case of the CoRoT database it was based on the visual inspection of density contour and scatter plots. The clumpiness of the distribution of data points in parameter space was somewhat less than for any of the other two experiments, suggesting a larger value of $\sigma$. We nevertheless decided to maintain the same value $\sigma=0.2$, given the unprecedented quality of the CoRoT data.

Plots of the clusters will be numbered and referred to according to the cluster identifier. 


\subsection{The Hipparcos archive}

The entire Hipparcos variability catalogue was processed as described in Debosscher et al. (2007), and light curves with $p$-values, as defined in Debosscher et al. (2009), below $10^{7}$ were retained for clustering analysis. Furthermore, we made use of class labels in order to interpret the resulting clusters. Figure 1 represents the 16 clusters found in this set of objects in the $\log (v)-(V-I)$ plane, using a value of the kernel width $\sigma=0.2$. Other projections of the original database using the other attributes can be found in Figs. 18-26. For the discussion of the Hipparcos database, we make use of the class labels from the General Catalogue of Variable Stars (Samus et al. 2002, GCVS) because this was also done by the Hipparcos team (Eyer \& Grenon 1997, e.g.).

From Fig. 1 we deduce that the algorithm separated long period (i.e., low frequency) variables of similar properties but with different behaviour in the values of $\phi_{12}$. Examples are clusters 7 and 10 which share similar properties and are only separated in the value of the $\phi_{12}$ of their modes. This is obviously an artefact due to the use of the quantity $\phi_{12}$ as an attribute, while this quantity is invariant with respect to $2 \pi$. The phase difference $\phi_{12}$ is only useful for a few classes, like RR Lyrae pulsators, Cepheids or eclipsing binaries because they show highly non-sinusoidal light curves. For long period variables (classes M, SR, I and L in the nomenclature of the GCVS) or low-amplitude pulsators, we find a more homogeneous coverage of the range of values of $\phi_{12}$, in agreement with the physics of their oscillations and their nearly sinusoidal variations in the light curve which implies a large uncertainty in the $R_{21}$ and $\phi_{12}$ values.

Further, the algorithm succeeds in the recovery of the most conspicuous groups of variables: cluster 1 contains most of the Cepheids in the Hipparcos catalogue, cluster 13 most of the RR Lyrae stars, cluster 4 the binaries of the EB type, and cluster 6 binaries of the EA and EB types. The Long Period Variables group (LPVs) includes as most numerous subgroups the Miras (M), Semiregular Variables (SR), Irregular Variables (I) and Slow Irregular Variables (L). These subtypes are spread over clusters 2, 5 and 12 (SR, I, and L), and 3, 7, 10, and 11 (Mira Variables with negligible contributions from other LPVs). While cluster 12 is characterized by periods of the order of several hundred days, clusters 2 and 5 show a much larger spread of periods down to a few days. Cluster 3 contains the lower-amplitude Miras, whose $\phi_{12}$-values are spread out compared to these for the Miras in clusters 7, 10, and 11. The latter are artifically divided due to the $2 \pi$ degeneracy in $\phi_{12}$ on the one hand and due to redder colour indices on the other hand.

We interpret the separation of Miras into four distinct clusters as the result of a finite sample, producing local low contrast maxima at random locations. A larger kernel density would smooth out these small overdensities at the expense of merging neighbouring clusters. The obvious solution to this is to cut the clustering dendrogram at different levels in each branch. Furthermore, this allows for the selection of relevant attributes in each branch, the necessity of which is exemplified by the segregation of groups introduced by an attribute such as $\phi_{12}$ which is not relevant for all classes of variables but very discriminating for others.

Cluster 14 contains most of the $\delta$ Sct stars in the sample while all other contributions to this cluster are small. The second most numerous contributing class are the SX Phe stars with only three objects. This is fully understandable from a physical point of view, as the SX Phe stars' variability behaviour is very similar to the high-amplitude $\delta$ Sct stars but they are old Population II stars instead (Rodriguez \& López-González 2000). Most other SX Phe stars constitute a satellite group of the RR Lyrae stars in cluster 13 (these two groups separate for smaller values of the kernel width) and are not assigned to the $\delta$ Sct cluster because they have larger amplitudes than the prototypical Population I $\delta$ Sct stars and only somewhat smaller than the Population II RR Lyrae stars (see Fig. 18). It is very assuring that the algorithm manages to distribute the SX Phe stars over the two other clusters whose physical properties they share.

Cluster 15 is composed of rather strictly periodic blue stars. These are pulsating stars of spectral type B belonging to the $\beta$ Cep, slowly pulsating B (SPB) and periodically variable supergiant (PVSG) classes discovered by means of multivariate discriminant analysis by Waelkens et al. (1998) and confirmed from ground-based follow-up data by Aerts (2000), Aerts et al. (1999), and Lefever et al. (2007). These stars are in general nonradial multiperiodic oscillators with low-order pressure $(\beta$ Cep) or gravity (SPB, PVSG) modes excited by the $\kappa$ mechanism. Their amplitudes are not too different from those of the $\delta$ Sct stars, even though some of the class members show somewhat more outspoken small nonlinear effects in their oscillations. Their periods are typically an order of magnitude longer than those of the $\delta$ Sct stars, as nicely illustrated in Fig. 1. The ground-based follow-up studies have shown that some of these candidate pulsating B stars turned out to be ellipsoidal binaries (De Cat et al. 2000) or spotted stars (Briquet et al. 2004) with periods similar to the oscillation periods of SPBs, but this was the case for a small minority only.

Cluster 9, on the other hand, contains $\gamma$ Dor stars. These are F-type multiperiodic stars pulsating in nonradial gravity modes with periods similar to those of the SPBs, i.e., also an order of magnitude longer than the $\delta$ Sct stars. They are situated on the red side of the classical instability strip. Numerous new class members were indeed found in the Hipparcos database by Aerts et al. (1998) and Handler (1999). Most of these were confirmed as class member later on, from extensive follow-up studies (Aerts et al. 2004; Mathias et al. 2004; De Cat et al. 2006) just as for the B-type pulsators discussed above. The amplitude and phase behaviour of the $\gamma$ Dor stars is similar to the one of the $\delta$ Sct, $\beta$ Cep and SPB stars, taking into account the $2 \pi$ degeneracy for $\phi_{12}$.

Finally, clusters 8 and 16 are dominated by so-called $\gamma$ Cassiopeiae stars (GCAS in the GCVS nomenclature). The star $\gamma$ Cas is a member of the classical Be stars, which are objects showing Balmer line emission in their spectrum due to the presence of a circumstellar disc (see Porter \& Rivinius 2003, for an extensive review on the observational and physical properties of those stars). This inhomogeneous class consists of both single and binary stars and, moreover, some Be stars show oscillations and/or light curve trends while others do not. It is thus normal that these objects are spread out over various clusters. Clusters 8 and 16 contain 32 and 17 such stars, respectively. The difference between the two cluster modes is only due to the phase difference $\phi_{12}$ of $2 \pi$, which is again artificial. Cluster 15 described above, also contains 9 Be stars according to the Hipparcos classification.

We conclude that our clustering analysis turned out to be a powerful method to separate the monoperiodic large-amplitude classical variables and binaries from the low-amplitude multiperiodic nonradial pulsators. Since these populations are well represented in the Hipparcos database of variables, we have shown our methodology to have the potential to recover all these classes in unexplored new databases as well, by means of the attributes that we have used here. Moreover, our analysis divided 


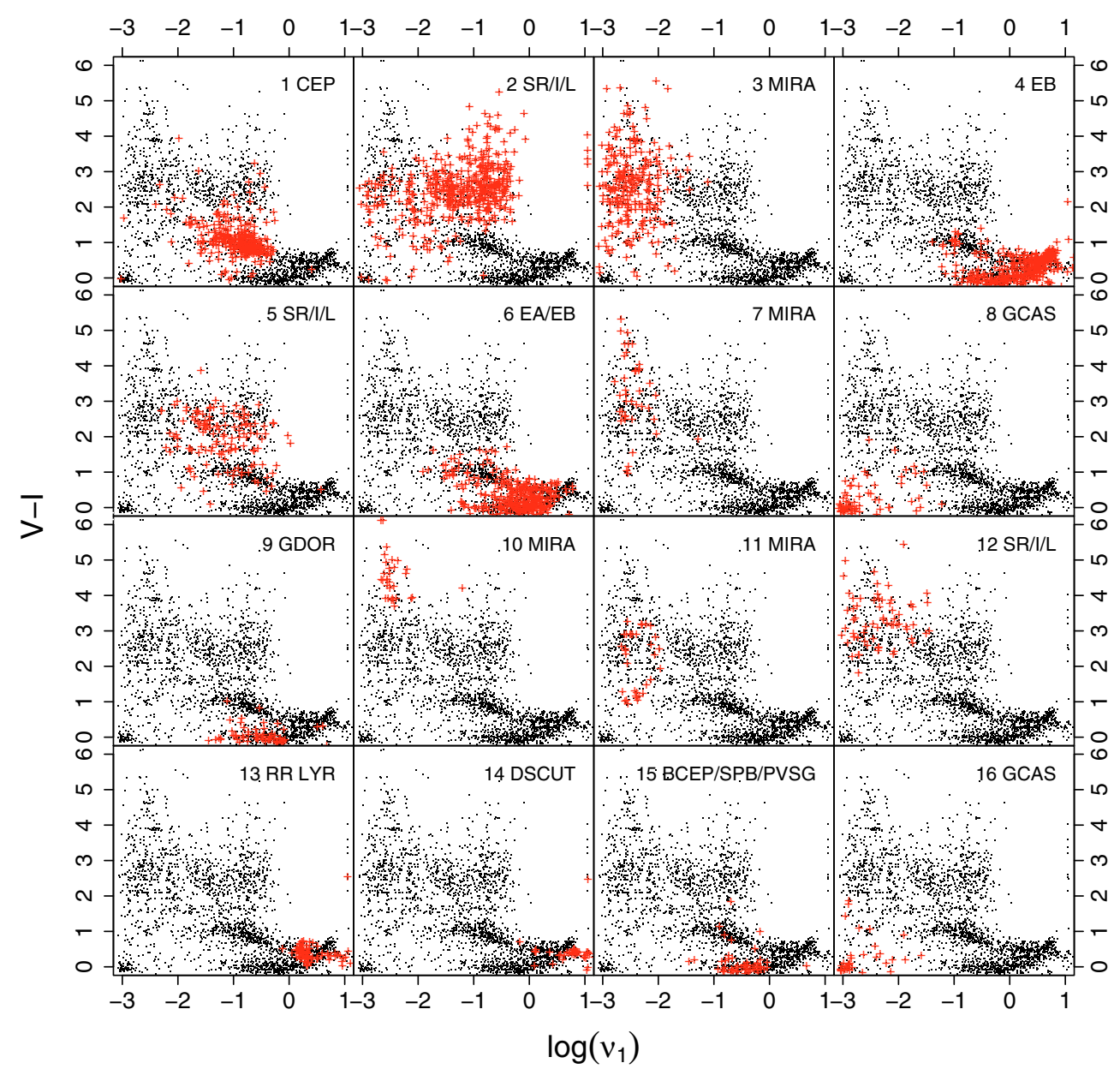

Fig. 1. The clustering structure of the Hipparcos variable stars archive at $\sigma=0.2$. The $x$-axis represents the logarithm of the frequency and the $y$-axis the $V-I$ colour index. Black dots represent the complete database and red crosses identify cluster members. Predominant classes according to the Hipparcos variability annex are included as labels in each box.

the four classes of nonradial pulsators along the main sequence, i.e., the best targets for asteroseismology (see Aerts et al. 2008, for a review), in four different clusters with the appropriate properties according to the physics of these stars.

\subsection{The OGLE archive}

The OGLE LMC variability database was processed in the same way as the Hipparcos archive and an equivalent dataset was generated with the same attributes. However, the phase difference $\phi_{12}$ was not used in the clustering experiments. While the use of $\phi_{12}$ was convenient to avoid a strong contamination of the RR Lyrae and Cepheids clusters by a particular type of eclipsing binaries for the Hipparcos database, the denser sampling (43 351 objects) defines the maxima of the density distribution better in the OGLE LMC case and diminishes (but does not eliminate) the contamination. Rather, the Wessenheit index $W_{I} \equiv I-1.55(V-I)$, which is a brightness indicator independent of extinction, was added in the analysis since this quantity has been used previously in the mining of the OGLE database.

The list of well identified clusters at the largest scales is similar to the one described above for the Hipparcos database, except that the OGLE data are less suited to find low-amplitude multiperiodic variables. This is illustrated in Fig. 2 in the frequency$W_{I}$ index plane, and further clarified in other projections in
Figs. 27-40. Amongst the largest clusters are those containing the First Overtone (FO) and Fundamental Mode (FU) Cepheids (clusters 7 and 8, respectively), and the RR Lyrae (cluster 6). The RR Lyrae cluster is further separated at smaller scales, into two large clusters corresponding i) to the ab subtype; and ii) to a mixture of the c subtype and double mode RR Lyrae pulsators (see Fig. 3), and several smaller clusters containing mainly spurious detections.

The Long Period Variables sequences defined by Soszynski et al. (2005) in the $W_{J K}-\log (P)$ plane (where the $W_{J K}$ index is the equivalent of the Wessenheit index for the $J$ and $K_{\mathrm{S}}$ near infrared photometric bands) are grouped into four large categories. The Long Period Variables region in Fig. 2 is shown again for clarity in Fig. 4. Each of these clusters is further separated into smaller groups at finer scale levels in the clustering hierarchy. In order of decreasing frequencies (increasing periods), the first and largest cluster comprises the A and B sequences (the so-called OGLE Small Amplitude Red Giant Stars, OSARGS); the adjacent cluster corresponds to the $C^{\prime}$ sequence (cluster 2; Mira and Semiregular variables) in the $W_{J K}-\log (P)$ plane. Figure 4 shows that this cluster can be separated into several groups at smaller bandwidth scales. The reason for this is that the unique $\mathrm{C}^{\prime}$ sequence in the $W_{J K}-\log (P)$ plane, splits into two when the Wessenheit index $W_{I}$ is used instead of $W_{J K}$, with each ridge characterized by a different slope corresponding to 


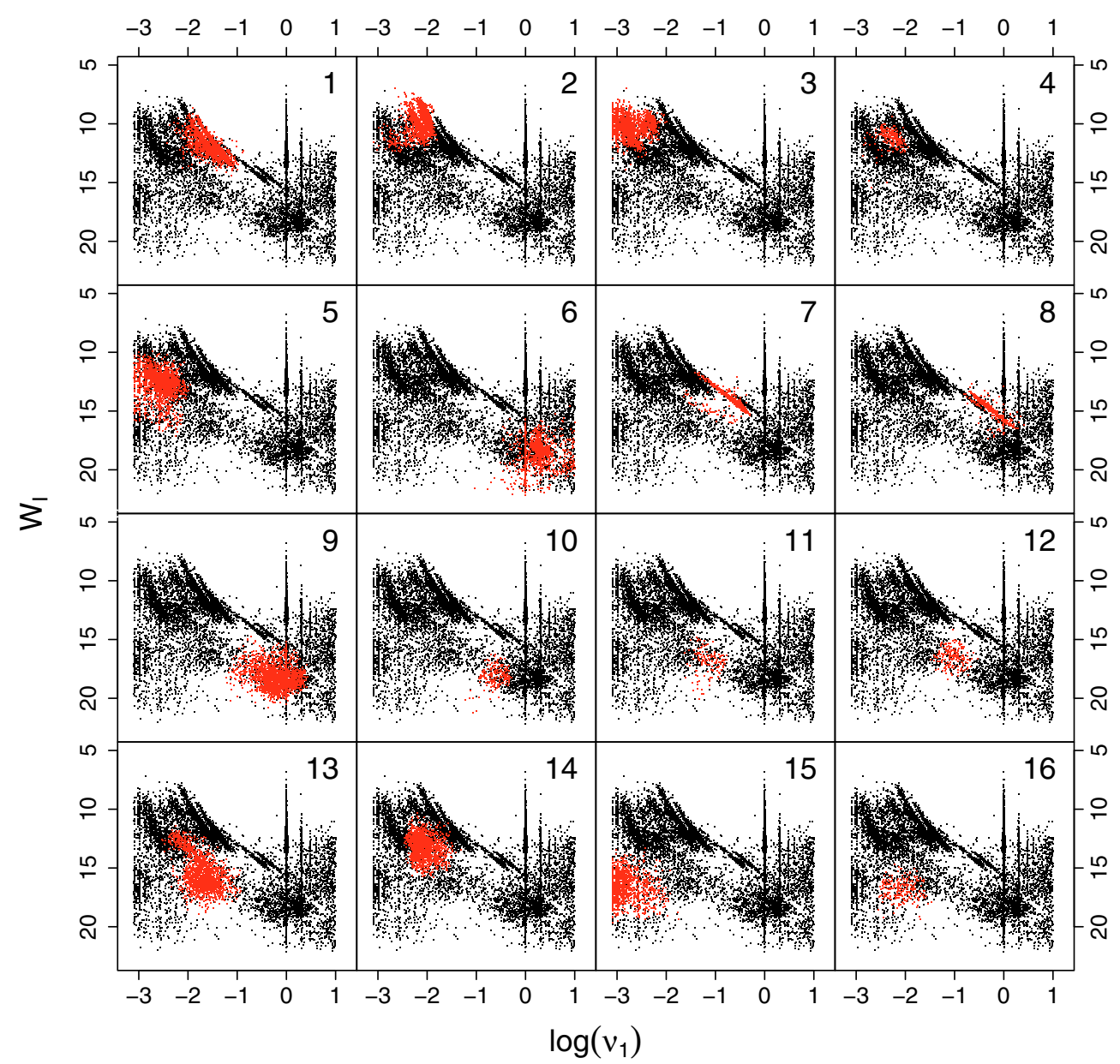

Fig. 2. The clustering structure of the OGLE LMC archive at $\sigma=0.15$. The $x$-axis represents the logarithm of the frequency and the $y$-axis, the Wessenheit index $W_{I}$. Black dots represent the complete database and red crosses identify cluster members. Note the occurrence of spurious frequencies at a value of one per day and multiples thereof due to the daily gaps in the data (one-day aliasing problem).

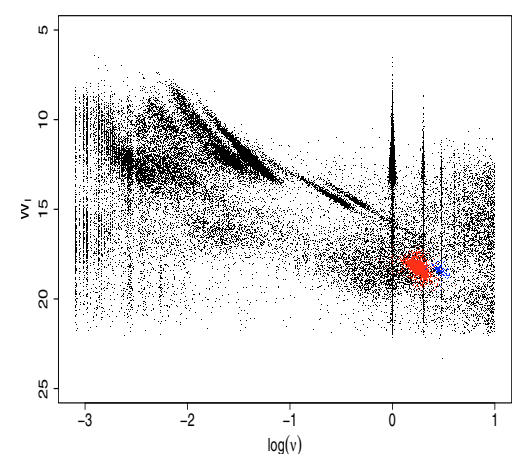

Fig. 3. The two largest subgroups in the RR Lyrae cluster, obtained with a smaller kernel bandwidth $(\sigma=0.1)$ than in Fig. 2. They correspond to the well known loci of the RRab (red dots) and RRc/RRd (blue dots) subtypes. The $x$-axis represents the logarithm of the frequency and the $y$-axis, the Wessenheit index $W_{I}$.

the different chemical composition of the stars (O-rich in the case of the larger frequency cluster and C-rich in the other cluster). The $\mathrm{C}$ sequence in the $W_{J K}-\log (P)$ plane is barely visible when the Wessenheit index is used, but the algorithm detects a cluster (cluster 3 ) that coincides with the descriptions found in Soszynski et al. (2005), except for the fact that it also contains objects in the $\mathrm{D}$ sequence (those with redder $V-I$ colours and larger amplitudes). Cluster 4 also seems to correspond to objects in the $\mathrm{C}$ sequence. Finally, the D sequence is clearly visible at

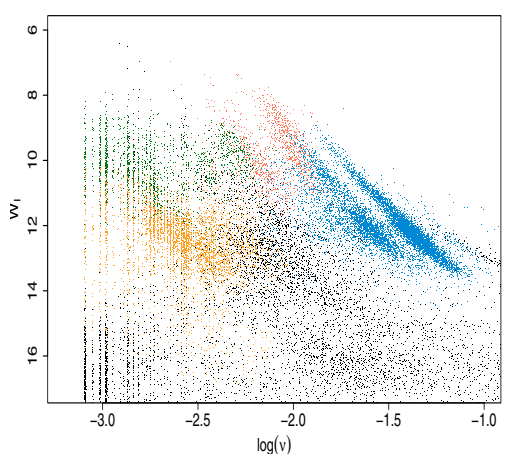

Fig. 4. The clustering structure of the OGLE LMC archive at $\sigma=0.15$, in the Long Period Variables region. The $x$-axis represents the logarithm of the frequency and the $y$-axis, the Wessenheit index $W_{I}$. Blue dots represent the A and B sequences (cluster 1), red dots represent the $\mathrm{C}^{\prime}$ sequence (cluster 2), dark green corresponds to the $\mathrm{C}$ sequence and the upper part of the D sequence (cluster 3 ) and orange represents the D sequence (cluster 4).

the smallest frequencies. As mentioned above, these groups (obtained with a large kernel bandwidth) are further split into the well known Long Period Variables sequences in subsequent levels of the hierarchy obtained with smaller kernel bandwidths.

The eclipsing binaries and ellipsoidal variables are gathered in 5 clusters (clusters 9 to 13). Cluster 9 corresponds to detached binaries of the EA and EB types, with flat light curves between 


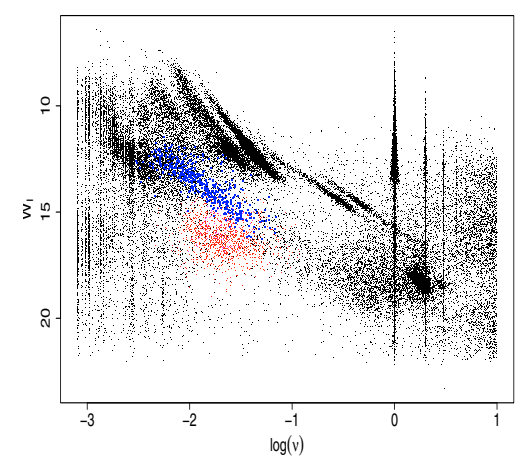

Fig. 5. The clustering structure of the OGLE LMC archive at $\sigma=0.3$ (after renormalization to the unit hypercube) for cluster 10. The $x$-axis represents the logarithm of the frequency and the $y$-axis, the Wessenheit index $W_{I}$. It clearly shows how the cluster is divided into two subgroups corresponding to the locus of ellipsoidal variables (blue) and EW eclipsing binaries (red).

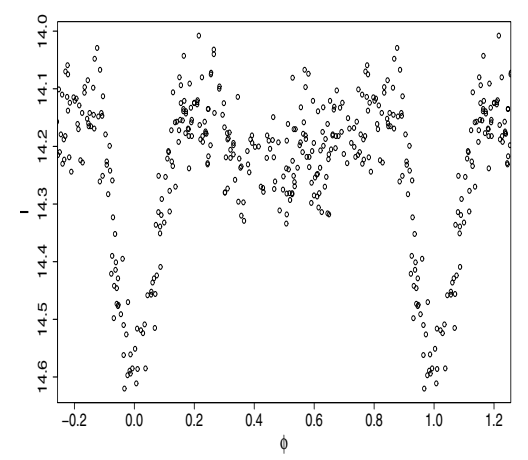

Fig. 6. Example of an ellipsoidal variable in an eccentric system (cluster 13b). The light curve qualitatively resembles the synthetic light curve computed for an eccentricity value $e=0.2$ and periastron length $p=90$ deg shown in Fig. 8 of Soszynski et al. (2004).

eclipses. As we go from cluster 9 to cluster 12 , we see a sequence in the shapes of the eclipsing binaries light curves, in the sense that the eclipse widths increase until there no longer exists a flat part of the light curve between eclipses. Figure 41 shows several examples of the light curves of objects closest to the corresponding cluster modes. Following the results obtained by Sarro et al. (2009) regarding the classification of hot mainsequence pulsators, some of these objects may have ended up in these binary star clusters.

Cluster 13 contains two subclusters that are easily separated at smaller kernel bandwidths (see Fig. 5). One of them (cluster 13a shown in blue in Fig. 5) shows a clear correlation between the frequency and the Wessenheit index, in agreement with the slope found by Soszynski et al. (2004), while cluster $13 \mathrm{~b}$ (shown in red in Fig. 5) shows no correlation in the $\log (v)-W_{I}$ plane. Visual inspection of the light curves in this cluster seems to indicate a predominant component of EW systems, although we also find eccentric ellipsoidals such as the one shown in Fig. 6. The comparison of clusters 13a and 14 with the population of variables discussed by Soszynski et al. (2004) seems to suggest that these two clusters are mainly composed of ellipsoidal variables in binary systems.

The last two clusters in Fig. 2 correspond to systems occupying the same region in the parameter space where the Hipparcos Be stars are found and correspond with stars having long periods and blue colours in the LMC sample (see Fig. 30). Visual inspection of the light curves of systems in the neighbourhood of the cluster mode indicates the presence of nonperiodic behaviour with long time scales in cluster 15 and a mixture of nonperiodic and strictly periodic behaviour in cluster 16 .

Additional clusters not shown in Fig. 2 show interesting properties, worth further investigation. One of them (shown in Fig. 7) contains a selection of candidates to the category of low amplitude periodic variables (Debosscher et al. 2009). This figure shows their position in two $2 \mathrm{D}$ projections of the parameter space, together with the fundamental and first overtone Cepheids loci. Two of the candidates (SC4 323401 and SC3 35239) belong to the list of ultra low amplitude Cepheids in Buchler et al. (2005). Other may again represent some of the candidate slowly pulsating B stars found from supervised classification in Sarro et al. (2009).

Another cluster, shown in the $\log (v)-(V-I)$ plane in Fig. 8, splits into several subclusters at smaller kernel bandwidths, two of which seem to group stars with properties typical of the $\delta$ Sct (shown as red dots) and $\beta$ Cep (in blue) types, respectively. These correspond to some of the stars identified as such type of mainsequence nonradial pulsators in Debosscher et al. (2009).

Finally, the cluster shown in Fig. 9 is located in the region occupied by the various sequences of red giant variables discussed in Soszynski et al. (2005) (see their Fig. 3), but does not correspond to any of the sequences discussed therein. It shows a remarkable correlation in the $\log (v)-(V-I)$ plane that seems to continue the sequence of $\mathrm{C}$-rich red giants in the $\mathrm{C}$ sequence, although there also exists the possibility that it represents a subpopulation of ellipsoidal variables (shown in blue in Fig. 9).

We conclude to find reasonable agreement between the extractor-type and supervised classification results applied to the OGLE LMC database and our clustering results. The majority of variables in the OGLE database are clearly large-amplitude monoperiodic pulsators or binaries, but we do recover evidence for the occurrence of some low-amplitude variables.

\subsection{The CoRoT intermediate archive}

In this section we show the results of the clustering analysis of the first four runs of CoRoT exoplanet data (IRa01, LRa01, LRc01 and SRc01; see Debosscher et al. 2009, for a description of the data). The goal of the exoplanet programme of the CoRoT mission is to detect planets around stars through the transit method (see Auvergne et al. 2009, for more explanation). In order to achieve this, light curves of thousands of stars with a precision typically a factor 100 better than the Hipparcos and OGLE data have been gathered during long uninterrupted sequences ( 5 months for a long run, a few weeks for a short run). The probability of finding exoplanets is much higher for mainsequence stars than for evolved stars. As such, the CoRoT exoplanet database is heavily biased towards main-sequence stars while giants and supergiants have been avoided in the preselection of the targets as much as possible. It is therefore evident that the population of variable stars in the CoRoT and OGLE databases are almost opposite in nature, the Hipparcos variable star database bridging these two extremes.

The analysis of the CoRoT dataset also differs from the previous two ones in that no colour information was used in the analysis. The CoRoT satellite is equipped with a dispersing prism that provides information on colour changes with time for a given star, but the masks are adapted to each of the stars separately and hence this information cannot be translated into any kind of standard colour index. Although there are groundbased colour indices available for a fraction of the archive from the CoRoT EXODAT database (Meunier et al. 2007), 

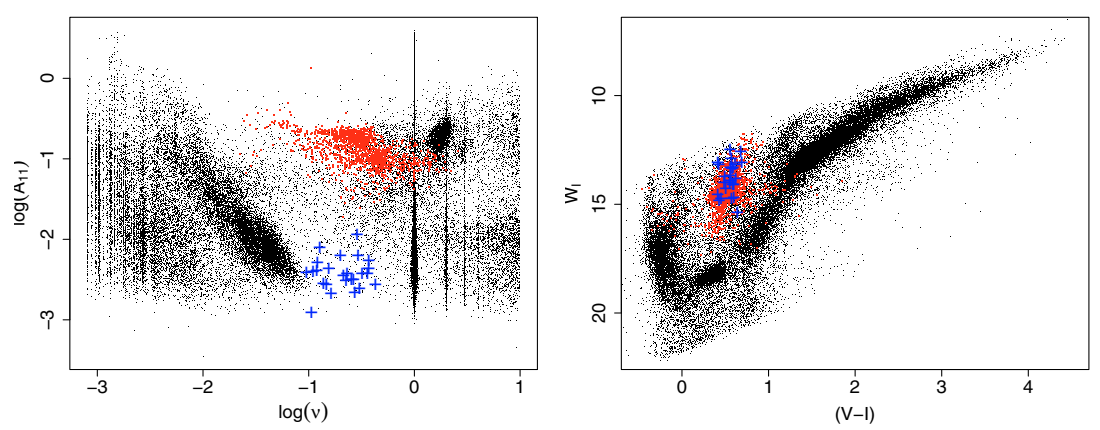

Fig. 7. Low amplitude periodic variable candidates in two dimensional projections of the parameter space. In the left plot, the $x$ axis represents the logarithm of the frequency, and the $y$ axis the logarithm of the amplitude of the first harmonic component of the Fourier decomposition. In the right hand plot, the $x$ axis represents the $V-I$ colour index, and the $y$ axis the Wessenheit index $W_{I}$. In both plots, classical Cepheids pulsating in the fundamental mode or the first overtone are represented with red dots, and the low amplitude candidates with blue crosses.

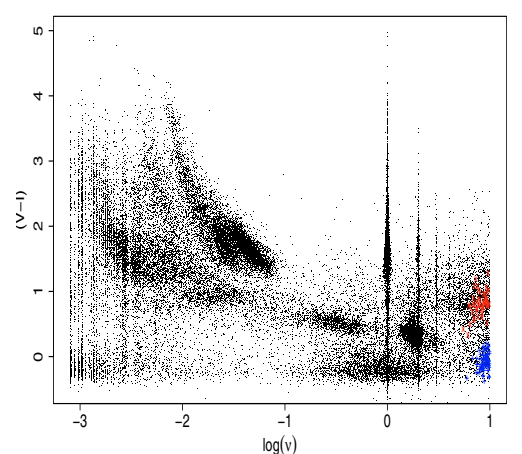

Fig. 8. Blue plus signs and red crosses represent the small scale structure of one of the clusters found, not shown in Fig. 2. The $x$ axis corresponds to the logarithm of the frequency $v$, and the $y$ axis, to the $V-I$ colour index.

we preferred not to use them for the clustering because they have not been corrected for reddening. This is also the case for the 2MASS (Skrutskie et al. 2006) colour indices. Although reddening effects should be less important in the infrared passbands, it has to be recalled that the CoRoT fields are located near the Galactic center (LRc01 and SRc01) and anticenter (IRa01, LRa01; see the 2MASS Explanatory Supplement to the Second Incremental Data Release for a comparison between typical colour-colour diagrams in Galactic plane and pole regions). Nevertheless, we have conducted a parallel experiment (described in Sect. 4.3.1 below) in order to assess the robustness of our results when 2MASS colours are used as attributes for clustering. Also, we would like the results of the clustering analysis to be used directly in the update and improvement of the supervised classification system which is based solely on CoRoT light curves (Debosscher et al. 2009). The lack of colour information will necessarily cause confusion among variability classes whose light curves are very similar. This is the case, e.g., for $\beta$ Cep and $\delta$ Sct stars, for SPBs and $\gamma$ Dor stars, and for various classes of stars with activity. We thus expect these to be difficult to separate.

On the other hand, the lack of colour information is partially compensated for by the unprecedented quality of the spectral frequency information that can be recovered from time series analysis of the CoRoT exoplanet light curves. For most of the light curves, numerous frequencies at low amplitudes are detected and thus we may hope to discriminate easily between monoperiodic and multiperiodic variability. This is in contrast to the Hipparcos and OGLE light curves, which quite often show only one

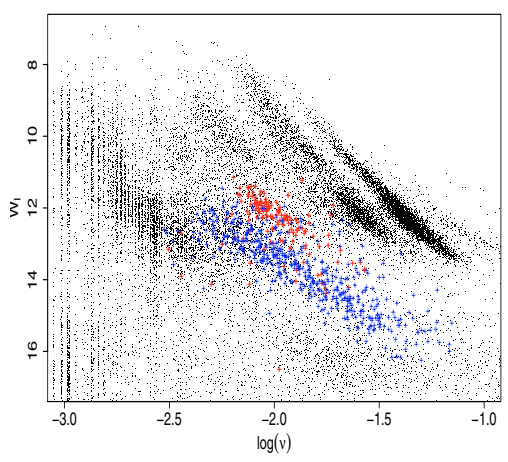

Fig. 9. Red crosses represent stars in one of the clusters obtained, showing a strong correlation in the $\log (v)-W_{I}$ plane. The $x$ axis corresponds to the logarithm of the frequency $v$, and the $y$ axis, to the Wessenheit index $W_{I}$. The region shown corresponds to the red giant sequences, and blue crosses correspond to the cluster interpreted as ellipsoidal variables in the text (shown here for comparison).

significant frequency. Hence, for the CoRoT database, it is most instructive to look at the clustering structure in the $\left(v_{1}, v_{2}\right)$ and $\left(v_{1}, A_{11}\right)$ planes (Figs. 10 and 11). We adapted the frequency threshold $\log \left(v_{1}\right)>-1.2$ as set in Debosscher et al. (2009) in order to avoid long-term trends (of intrinsic or instrumental nature) to dominate the frequency derivation. We clearly see in Fig. 10 that many stars reside along the bisectors in the subpanels, which points towards multiperiodic variables. The most conspicuous OGLE clusters corresponding to the monoperiodic RR Lyrae stars, Cepheids, eclipsing binaries and small amplitude red giants loci (more than $90 \%$ of the OGLE variables) are absent here. The implications for the supervised classification of these targets is straightforward: the performance of a classifier optimized for the OGLE sample of variable stars should not be maintained when applied to such a different stellar population. This is why the training set has been filled with CoRoT stars as much as possible for the supervised classification in Debosscher et al. (2009).

The interpretation of the clusters shown in Figs. 10 and 11, as well as in Figs. $42-48$ is again based on their position in the various projections of the parameter space, and also on the visual inspection of the first tens or hundreds of time series and phase-folded light curves. As already emphasized, we expect the interpretation of the clusters to be more difficult than for the Hipparcos and OGLE cases, as the most prominent clusters for these databases are absent here and as we expect groups 


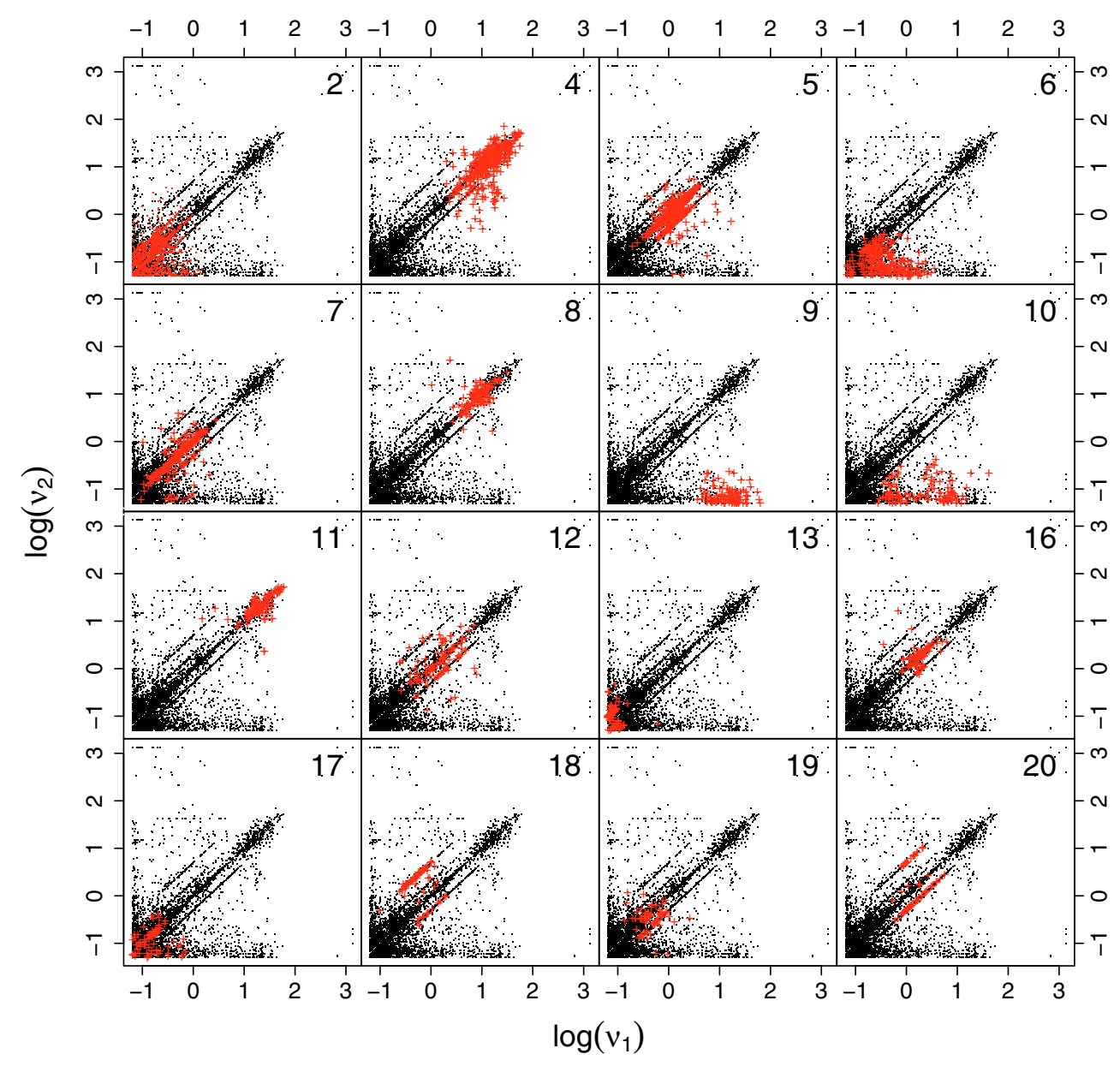

Fig. 10. The clustering structure of the CoRoT first four runs archive at $\sigma=0.2$. The $x$-axis represents the logarithm of the first frequency and the $y$-axis, the logarithm of the second detected frequency. Black dots represent the complete database and red crosses identify cluster members.

of stars with similar periods to be mixed due to lack of colour information.

The clusters shown in Figs. 10 and 11 are sixteen of the largest clusters found, although many less populated yet still significant clusters are found beyond these. The reader will have noticed from the subpanel labels that clusters 1, 3, 14 and 15 are absent. Visual inspection of their light curves showed that these clusters contain mostly spurious frequency detections due to jumps in the light curves as a consequence of hot pixels spikes due to the passage of the satellite through the South Atlantic Anomaly. These effects are not intrinsic to the stars, which is why these clusters are not discussed here. For the time being we have no appropriate solution to this problem, although automated jump correction and detrending methods as in Degroote et al. (2009) seem a promising route for future applications before the clustering analysis. We realise that these instrumental artifacts sometimes occur together with underlying low-amplitude periodic signal and that the latter unfortunately is masked by the jumps in the time series or vice versa. We thus expect our retained clusters to be possibly contaminated by light curves with small instrumental jumps, particularly for the clusters with periodicities of the order of days.

Let us look at the low-amplitude multiperiodic oscillators first. As already mentioned, we expect them to populate the bisector regions in Fig. 10, well above the point $\left(\log v_{1}, \log v_{2}\right)=$ $(-1,-1)$. This is the case for clusters $4,5,8,11,16$, and less clearly also clusters 7,12 , and 19 . Cluster 4,8 , and 11 clearly cover the periodicities of the $\beta \mathrm{Cep} / \delta$ Sct range, while clusters 5 ,
7,12 , and 16 correspond to frequencies in the $\mathrm{SPB} / \gamma$ Dor range. The dominant frequency of the cluster 9 stars is also $\delta$ Sct-like. The class labels obtained with the supervised classifiers presented in Debosscher et al. (2009) generally agree with these cluster identifications based on their position in parameter space.

Based on the Initial Mass Function, $\delta$ Sct stars will predominate in clusters $4,8,9,11$ over the $\beta$ Cep population (a hypothesis not contradicted by the unreddened EXODAT colours). Clusters 4 and 9 gather the vast majority of $\delta$ Sct stars $(423+$ 112 stars) in the first four runs. The clusters 8 and 11 contain 117 and 103 stars respectively and they show distinctive features in several projections of the parameter space. Cluster 8 is characterized by lower frequencies, larger amplitudes and larger $R_{21}$ ratios than clusters 4 and 9 . We interprete this as the subgroup of $\delta$ Sct stars with nonlinear light curve distortion due to high-amplitude (non)radial modes. Cluster 11, on the contrary, seems to contain the $\delta$ Sct stars with extremely low amplitudes. Cluster 9 is interpreted as the group of $\delta$ Sct stars whose second frequency is very low. These are probably hybrid $\delta \mathrm{Sct} / \gamma$ Dor stars, $\delta$ Sct stars with rotational modulation, or else $\delta$ Sct stars in binaries with grazing eclipses or ellipsoidal variability. The latter two options are less likely given that there is no specific phase relation for the second frequency (Fig. 49). On the other hand, these stars clearly have a higher value of $A_{22}$ (Fig. 46) and $R_{21}$ (Fig. 47) compared to those quantities for cluster 4 stars, pointing towards deviations from sinusoidal light variability with frequency $v_{2}$. This behaviour of $v_{2}$ can be interpreted in terms of rotational modulation or of nonlinear g-mode oscillations triggered 


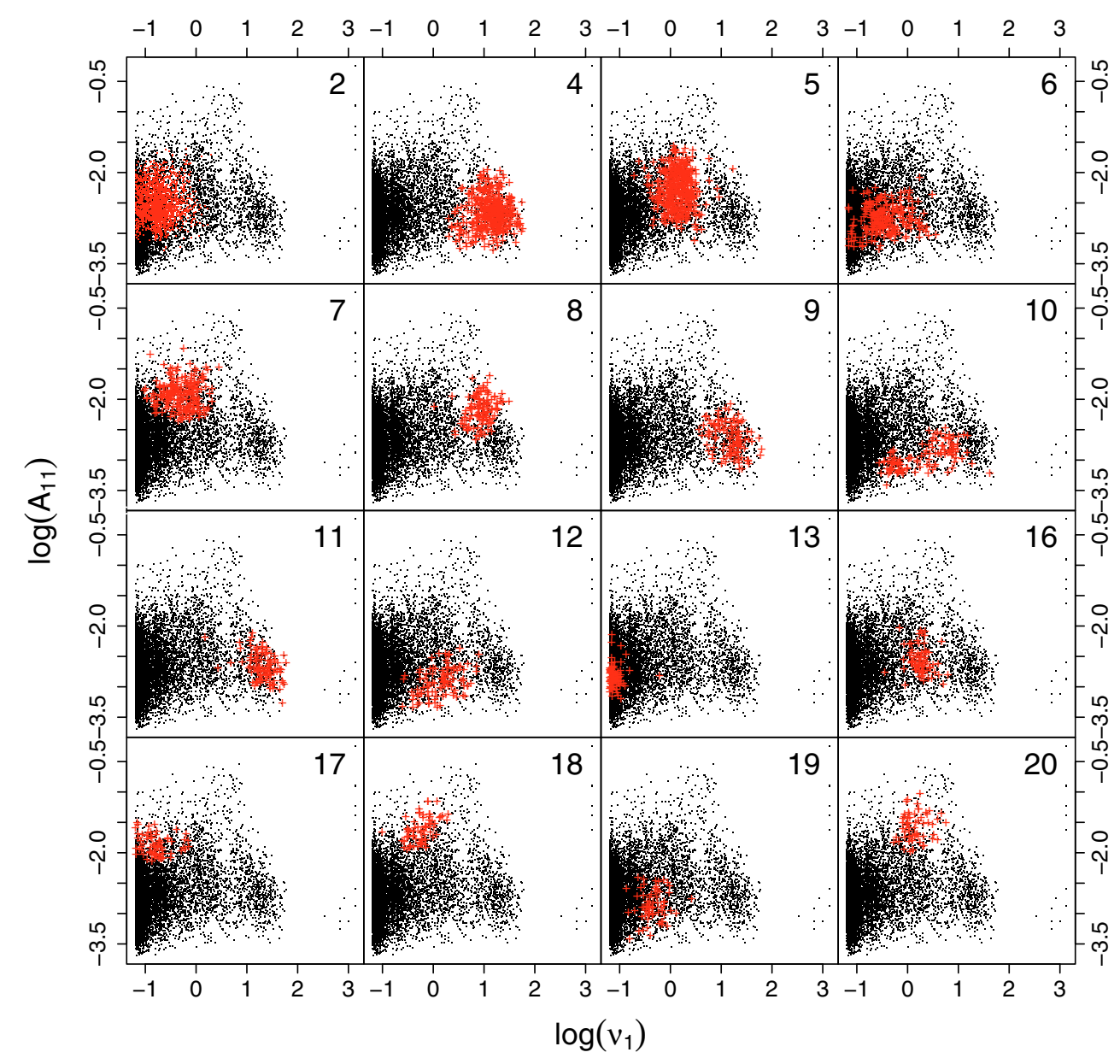

Fig. 11. The clustering structure of the CoRot archive at $\sigma=0.2$. The $x$-axis represents the logarithm of the frequency and the $y$-axis, the logarithm of the amplitude $A_{11}$ in magnitudes of the first component in the Fourier decomposition for $v_{1}$. Black dots represent the complete database and red crosses identify cluster members.

by resonant mode coupling (Buchler et al. 1997). It is very difficult to discriminate between these two scenarios without spectroscopic information, particularly since g modes may also be splitted into multiplets by rotational effects on the oscillations.

The clusters 8 and 11 show a clear bimodality in the unreddened colour, $v_{1}$ diagram as shown in Fig. 12, where the stars of cluster 11 turn out to be somewhat bluer and those of cluster 8 redder than average. This independent information clearly suggests that there is a physical reason why these clusters were found as separate classes, rather than this being due to statistical noise in the point process. In fact, the low-amplitude $\delta$ Sct stars in cluster 11 seem to coincide with the low-amplitude pulsators filling the gap between the classical instability strip and the SPB strip found by Degroote et al. (2009) which explains why they are somewhat bluer than the majority of the $\delta$ Sct stars. On the other hand, the stars in cluster 8 have clearly longer periods and are redder, while their amplitudes are also somewhat higher than average. This frequency-colour behaviour indicates that they are rather evolved $\delta$ Sct stars. The time series of the objects closest to the clusters modes are shown in Fig. 13 and fully confirm our interpretation.

The differences amongst clusters $5,7,12$, and 16 are mainly related to the amplitudes of the first frequency. As shown in Fig. 11, cluster 7 corresponds to the largest amplitudes, cluster 5 stars also have relatively high amplitude while cluster 12 and 16 shows significantly smaller amplitudes, and cluster 16 also somewhat higher frequencies. Some of the light curves in the vicinity of the mode of clusters 12 and 16 have low signalto-noise ratios. Moreover, cluster 16 is not only characterized by higher mean frequencies, but also by lower mean values of $R_{21}$, which implies that the oscillations in that group of stars is highly linear, while modest nonlinear effects seem to occur for the stars in cluster 5,7, and 12. All these properties suggest that clusters $5,7,12$, and 16 contain SPB and $\gamma$ Dor stars, which are both classes of multiperiodic gravity-mode nonradial oscillators. The time series of the four objects closest to the cluster 5 (top left), 7 (bottom left), 12 (top right) and cluster 16 (bottom right) modes are shown in Fig. 14 and support this interpretation.

The position of the stars in these four clusters in the unreddened colour diagram shown in Fig. 12 seems to suggest that the clusters 5, 12, and 16 contain a mixture of SPB and $\gamma$ Dor stars, with the majority being $\gamma$ Dor stars, again based on the Initial Mass Function. The stars have essentially the same colour properties, which confirms that the bluer SPBs must be relatively low in number than the redder $\gamma$ Dor stars. The stars in cluster 7 are clearly redder and have longer periods connected with their higher amplitudes. Again, we interprete this as an evolutionary effect and thus this cluster contains the evolved $\gamma$ Dor stars. A striking feature in Fig. 12 are some ten stars of cluster 12 and one of cluster 7 with far redder colours than all others in the four clusters with g-mode pulsators. While we have to be careful for overinterpretation of Fig. 12 due to lack of an appropriate correction for reddening effects, it seems to suggest that these stars are much more evolved than the other g-mode pulsators. It 


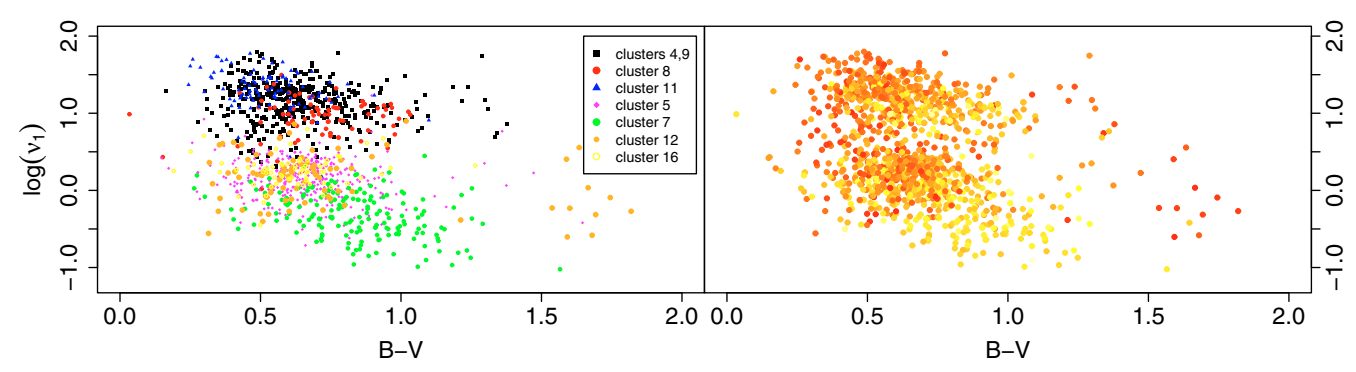

Fig. 12. Frequency-colour diagrams of the low frequency multiperiodic pulsators clusters. The left plot shows clusters 4 and 9 (black filled squares), 8 (red circles), 11 (blue triangles), 5 (magenta diamonds), 7 (green circles), 12 (filled orange) and 16 (open orange). The right panel shows the same plot with the logarithm of the amplitude of the first Fourier component colour coded from red (low amplitudes) to yellow (high amplitudes). The $x$ axis represents the EXODAT $B-V$ colour index, and the $y$ axis, the logarithm of the first detected frequency.

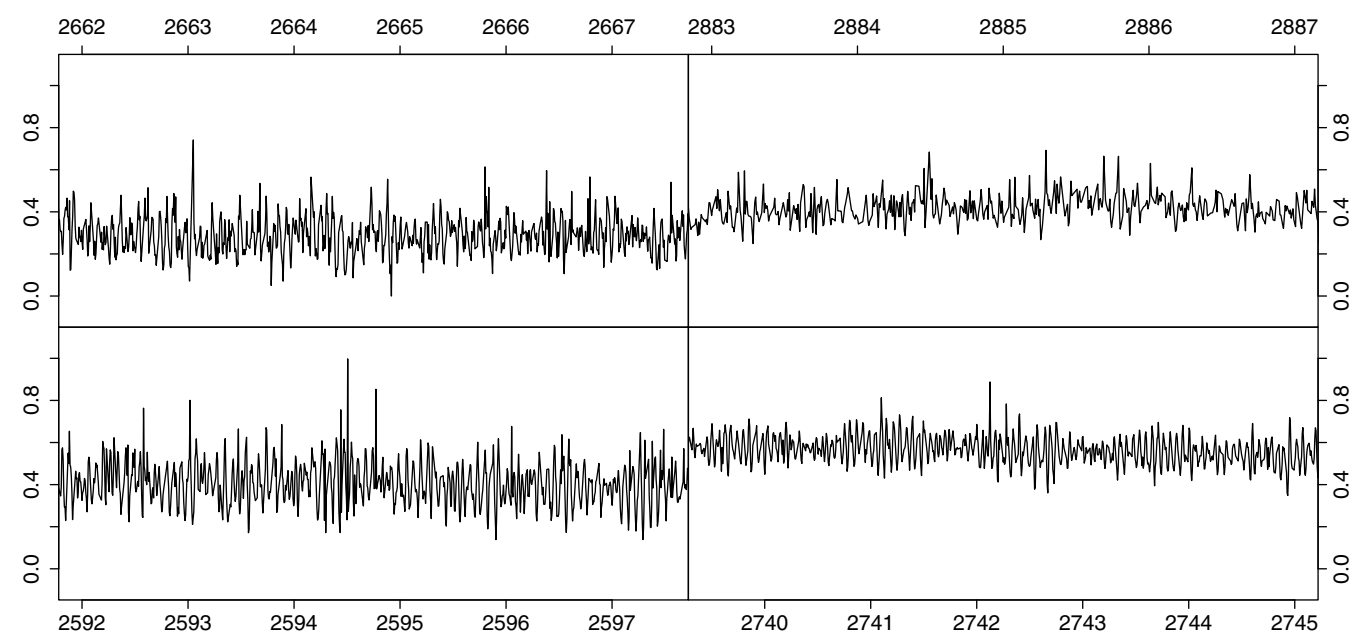

Fig. 13. Close-up of the CoRoT photometric time series of the object closest to clusters 4 (top left), 8 (bottom left), 9 (top right), and 11 (bottom right). The $x$ axis represents the modified heliocentric Julian Date, and the $y$ axis, the signal measured in counts.

could be that this small group of stars represent the PVSG class, which are evolved B stars with SPB like oscillations with longer periods (Lefever et al. 2007).

We cannot exclude that both cluster 8 and 7 are contaminated by a few pre-main-sequence $\delta$ Sct and $\gamma$ Dor stars, respectively, which would still be surrounded by remnant material of their birth cloud and which would be an alternative explanation for their redder colour.

Finally, we point out that the frequency - colour behaviour displayed in Fig. 12 is in full agreement with the theoretical predictions of the instability strips of the $\delta$ Sct and $\gamma$ Dor stars, along with a small number of pulsating B stars, as presented in, e.g., Degroote et al. (2009).

Several clusters contain stars whose variability patterns we interprete in terms of phenomena related to stellar activity. The largest cluster (cluster 2) is composed (again, according to the time series of the first tens of objects closest to the cluster mode) of stars showing time series characterized by the occurrence of harmonics of the dominant frequencies, which points to strongly non-sinusoidal light curves. Often, this can be explained in terms of one or several starspots, that can migrate in phase with respect to one another. A typical light curve corresponding to the objects closest to the cluster mode is shown in Fig. 15.

Cluster 17 contains the most extreme examples of strict rotational modulation active in the stellar photospheres, in the sense of being characterized by some of the largest amplitudes in the CoRoT sample as well as very high $R_{21}$-values (see Figs. 47 and 52).
Clusters 6 and 10 show similar behaviour but with an interesting peculiarity: the variability of these stars is systematically described by two very different dominant frequencies (see Fig. 10). Cluster 6 shows clear signs of bimodality, something that is confirmed by clustering analysis with smaller kernel bandwidths: it is composed of two populations, one with similar values of the first two detected frequencies, interpreted as complex activity, and one characterized by values of $\log \left(v_{1}\right)$ between -1 and 1 , and typical values of $\log \left(v_{2}\right)$ less than -1.0 . The latter stars are interpreted as active stars whose light curves also show long-term trends. Figure 50 shows close-ups of time series in the vicinity of the modes of cluster 6 . We can see very conspicuous low frequency modulations superposed to an activity signature.

Cluster 10 is also bimodal at smaller kernel bandwidths, but both subclusters are characterized by $\log \left(v_{2}\right)$ values around -1.0 , i.e., a long-term trends, while being bimodal in their $\log \left(v_{1}\right)$, with values around -0.15 and 0.8 , respectively. It may very well be that this groups consists of pulsating Be stars already discussed above in the framework of the Hipparcos database. These stars indeed undergo trends due to some level of activity and/or outbursts, while showing also oscillations with frequencies of pressure modes or gravity modes. Figure 51 show close-ups of time series in the vicinity of the modes of cluster 10 . These light curves are free from significant jumps. It has to be beared in mind that, in these clusters, it is the high frequency component that is detected first in the power spectrum, and it is thus associated to a higher peak. Future spectroscopic data are needed to refine this preliminary interpretation of cluster 10 . 


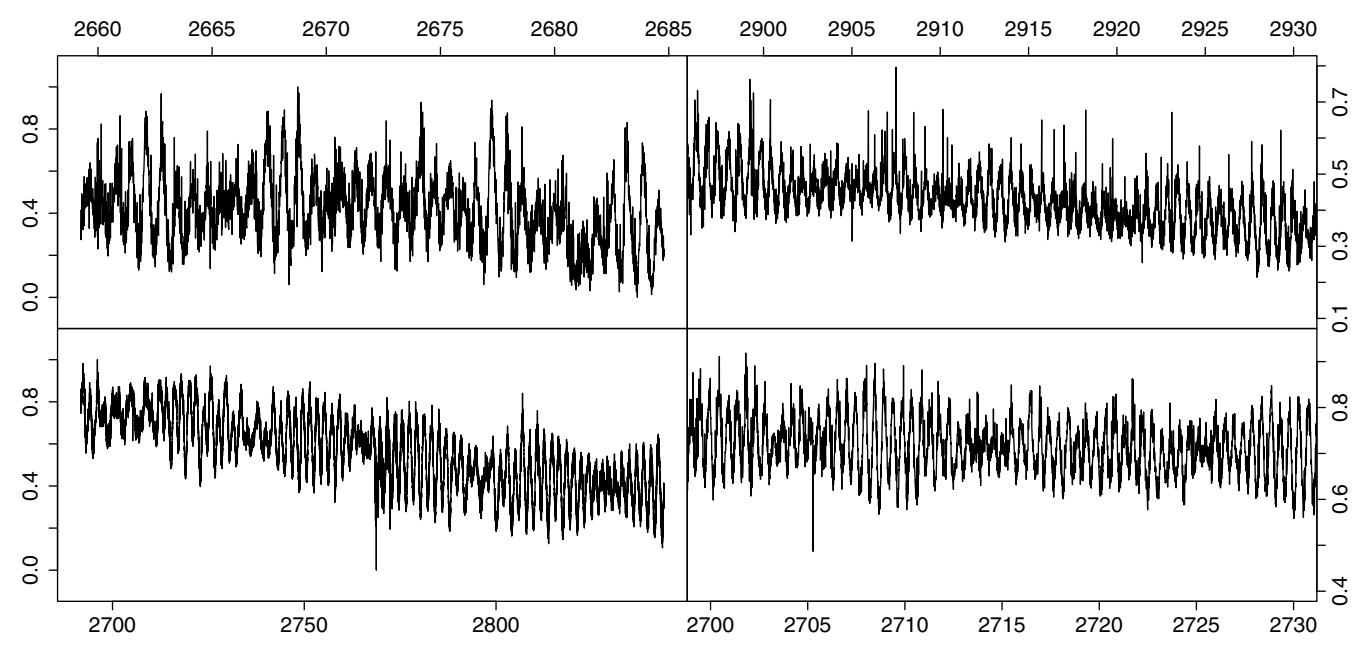

Fig. 14. Close-up of the CoRoT photometric time series of the object closest to clusters 5 (top left), 7 (bottom left), 12 (top right), and 16 (bottom right). The $x$ axis represents the modified heliocentric Julian Date, and the $y$ axis, the signal measured in counts.

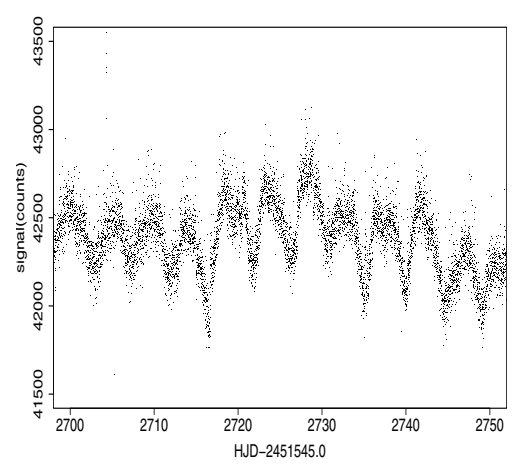

Fig. 15. Close-up of the CoRoT photometric time series of the object closest to the cluster 2 mode. The $x$ axis represents the modified heliocentric Julian Date, and the $y$ axis, the signal measured in counts.

The majority of eclipsing binaries are collected in clusters 18, 20 (and 21, and 22, plots not shown). This can be readily deduced from the phase behaviour of the dominant frequency as illustrated in Fig. 48. It turns out that the object closest to the mode of cluster 18 is a binary system (or a blend within the mask of CoRoT) with a multiperiodic component (see Fig. 53). In general, we found a few eclipsing binaries with pulsating components in the clusters with pulsators discussed above. The majority of stars in the four binary clusters 18, 20, 21, 22 only show eclipses, although some also have a signature of rotational modulation and/or activity outside the eclipses.

Finally, cluster 19 is a mixture of binaries, stars with activity, and some seemingly multiperiodic stars, while cluster 13 contains a mixture of stars with artificial jumps.

\subsubsection{Clustering with $2 \mathrm{MASS}$ colours}

We have conducted two complementary experiments in order to evaluate the information that the 2MASS colour indices could potentially provide to the clustering process. First, we analysed the clustering structure described above in the light of the infrared properties of the CoRoT targets (i.e., we retain the clusters found above and analyse the characteristic colours of the stars in these clusters). Figure 16 shows the colour-colour diagram of the CoRoT sample, with the cluster membership described in the previous section superimposed as red symbols.

It is evident from the plots that the reddening has smeared out the underlying distribution (see the Galactic pole colour-colour diagram in the Explanatory Supplement to the 2MASS Second Release). Nevertheless, Fig. 17 shows some interesting features that arise when the 2MASS colour indices are interpreted in the light of the frequency content of the light curves. First of all, contamination of cluster 2 by spurious frequency detections due to discontinuities in the time series is apparent as vertical lines in the corresponding plot. Second, the bimodality in frequency space of clusters 6 and 10 (described above) seems to correlate well with the $J-H$ colour index, in the sense that the subgroups with smaller frequencies correspond partly to a distinct group of redder, most probably giant stars.

In the second experiment, we carried out a new clustering of the CoRoT database, but this time we extended it with the 2MASS colour indices. We then studied the new groups of stars in relation with the clusters obtained without 2MASS data. The results can be summarized as follows:

- The clusters interpreted as $\delta$ Scuti and $\beta$ Cephei stars $(4,8$, 9 , and 11) remain as easily recognizable clusters in the new clustering structure. Cluster 9 is split into two clusters, and the other three are preserved, with a clear tendency for cluster 4 to absorb a significant fraction of clusters 8 and 11. The original differences between these clusters (described thoroughly in the previous section) seem to be smeared out by the use of the 2MASS colours. Little or no contamination is observed from other clusters;

- stars that belonged to the clusters interpreted as $\gamma$ Doradus and Slowly Pulsating B stars (5, 7, 12, and 16) remain grouped in a number of clearly identifiable clusters, and are not contaminated by other clusters. There is again a tendency for the clusters to merge into one larger cluster, except for cluster 7 that remains largely separated;

- clusters numbered 1,2 and 3 in the clustering experiment without 2MASS colours, remain the three largest groups when the latter are taken into account, but the contigency table (the table that summarizes the number of stars previously in cluster $i$ and now in cluster $j$ ) shows a major redistribution of stars in these categories. We interpret this as a hint that objects in clusters 1 and 3 should not be automatically 


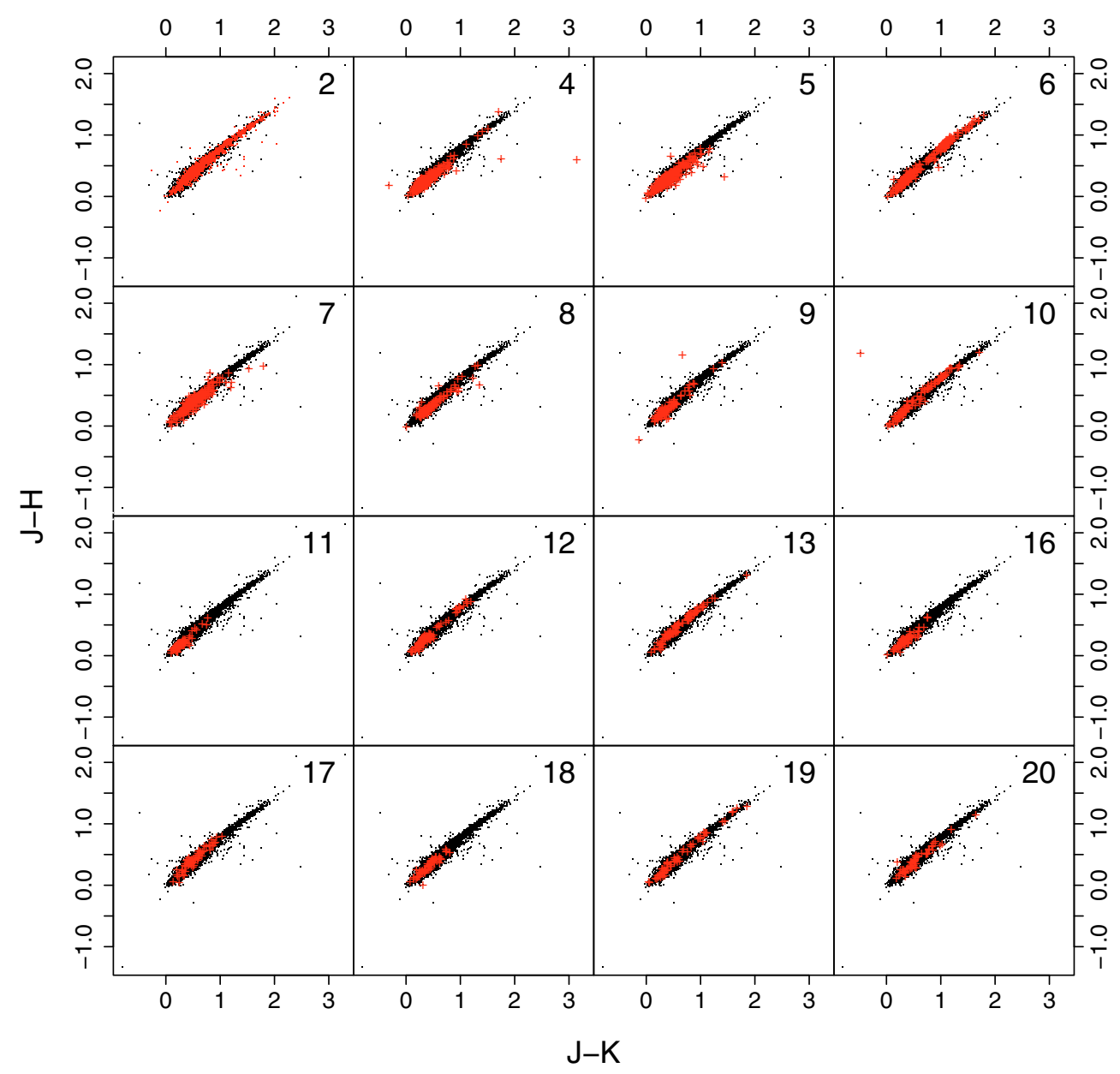

Fig. 16. 2MASS colour-colour diagrams of the CoRot clusters obtained with $\sigma=0.2$ and no colour index used as atribute. The $x$ and $y$-axis represent the $J-K$ and $J-H$ colour indices respectively. Black dots represent the entire sample and red symbols correspond to cluster members.

disregarded as "artifacts". Further studies are needed to better separate these from real signals of astrophysical origin;

- the clearly bimodal cluster 6 splits into two larger clusters (interpreted as dwarves and giants in the paragraph above) and two smaller ones; a similar behaviour is observed in cluster 10, with some stars merging into one of the smaller clusters originated in cluster 6 ;

- finally, the eclipsing binaries remain clearly separated as groups, with minor redistributions of stars in clusters.

\section{Conclusions}

We presented the results of extensive clustering experiments with three variability databases of very different characteristics, using a density based approach. We concentrated on the large scale clustering properties of the samples, trying to understand what domain of the variability zoo can be recovered with this kind of multivariate analysis. While the Hipparcos archive is characterized by a small number of objects with reliable class assignments which simplify the interpretation of the clusters, the CoRoT database is only starting to be understood due to the very high level of precision.

The results presented here already constitute a clear cut separation of variability types, as we understand them based on theoretical grounds. The clusters discussed above have been interpreted on the basis of their average properties, and on the visual inspection of those objects close to the clusters modes. Since these modes are located at the regions of maximum probability density, these are the prototypes of the clusters, but significant contamination from a variety of other objects cannot be discarded, and should, in fact, be assumed. This is mainly the case for those types where the attributes used to describe the objects are insufficient for a unique description of the time series. Nevertheless, the existence of well defined clusters containing several types of light curves with the imprint of nonradial oscillations, rotational modulation, activity, binarity, etc., in the CoRoT database, encourages the use and improvement of the current attribute set to interprete the detailed physics of the clusters.

We used the Hipparcos and OGLE LMC archives in order to define a reference frame that helps both the interpretation of the CoRoT database clustering structure and the discovery of new classes in it. In the Hipparcos archives, we clearly managed to separate the classical pulsators (Cepheids and RR Lyrae stars) from the eclipsing binaries and from the multiperiodic low-amplitude pulsators along the main sequence. This was successful thanks to the availability of a well-calibrated colour index. In the LMC case, the use of reddening free attributes like the Wessenheit index allows for the recovery of the various sequences of red giants in the long period range of the archive. Furthermore, the ellipsoidal variables are easily recognized in the OGLE clusters thanks to the $\log (v)-W_{I}$ correlation that they follow. 


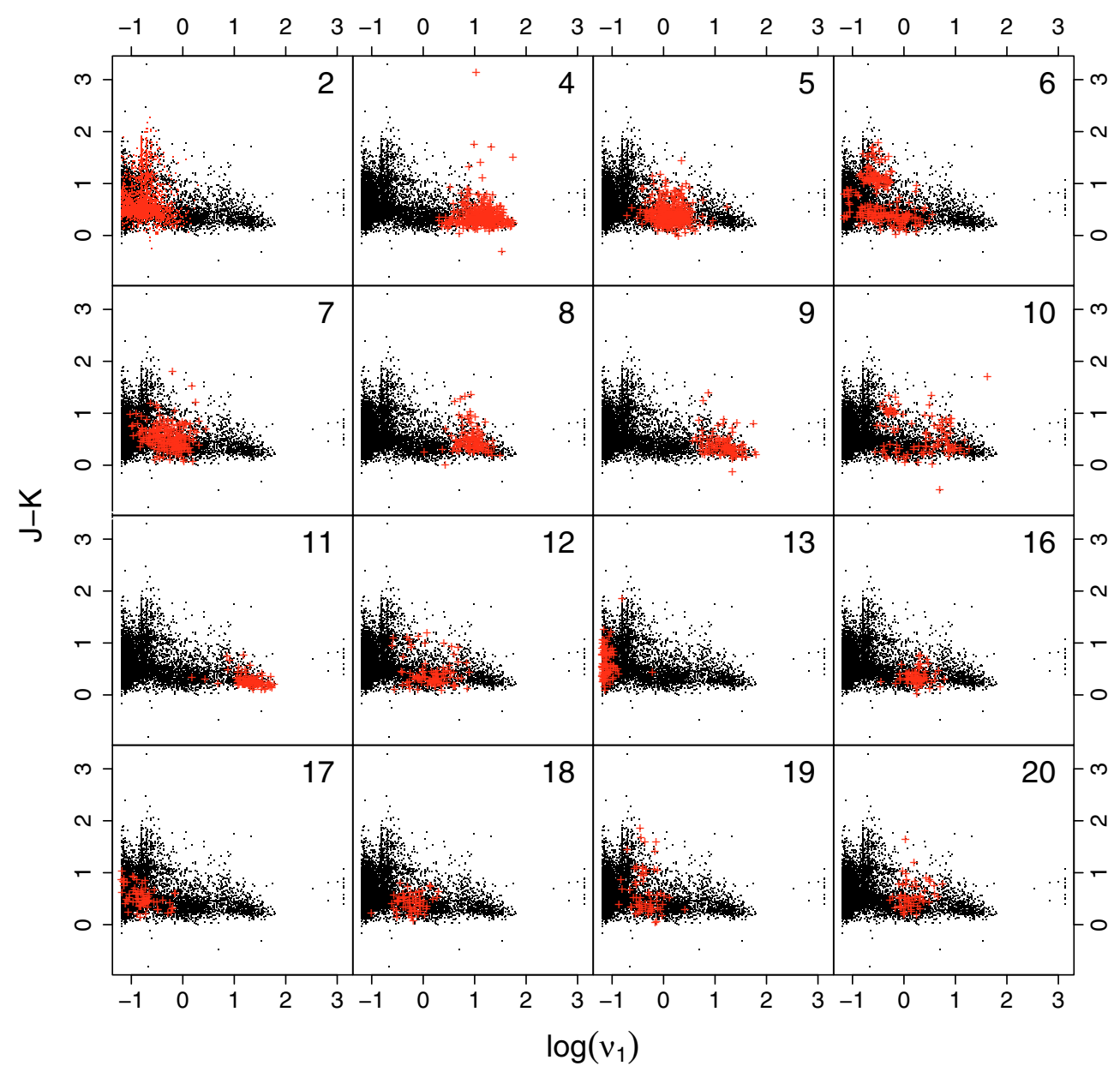

Fig. 17. The clustering structure of the CoRoT first four runs archive at $\sigma=0.2$. The $x$-axis represents the logarithm of the first frequency and the $y$-axis, the $J-K$ colour index. Black dots represent the entire sample and red symbols correspond to cluster members.

For the CoRoT exoplanet database, we have shown that we are capable of discovering various different properties among the low-amplitude multiperiodic stars. Although the confirmation of the various proposed subclasses among known nonradial pulsators and stars with activity must await the collection of complementary information, likely in the form of spectra, we are capable of discovering refinements of the properties of globally understood variability. Such refinements are only possible thanks to large scale clusters unravelled in the data.

The unprecedented quality of the time series provided by CoRoT has opened a completely new realm of variability, where low amplitude signals constitute new clusters by themselves, or appear as second or third frequencies combined with large amplitudes ones, sometimes giving rise to a separate cluster.

One of the most interesting findings, is the clear separability of the subpopulations among the previously known classes nonradial pulsators along the main sequence. Prior to the CoRoT mission, we had only been able to hint at the properties of larger versus lower amplitude members of the real distribution of these objects in paramater space, as well as on the suspicion of hybrid pulsators showing pressure and gravity modes simultaneously, for the $\beta \mathrm{Cep} / \mathrm{Be}$ versus SPB classes on the one hand, and for the $\delta$ Sct versus $\gamma$ Dor stars on the other hand. Also, the clustering pointed towards pulsating stars of various kinds in close binaries. Completely new sample of these pulsators will soon be publicly available to the scientific community for further studies, to be complemented with other sources of information.
All this knowledge gained with the unsupervised classification of the CoRoT database needs to be incorporated in the framework of the supervised classifiers, especially in the case of the light curve characteristics due to rotational modulation and stellar activity. These variables turn out to represent a very significant fraction of the database while they are not sufficiently well represented in the training set used for the supervised classification (Debosscher et al. 2009).

A systematic analysis of the smallest clusters as well as the decomposition into finer scales is left for future work. In the case of the CoRoT archive, this further analysis will be complemented with data from new runs, enhancing seriously the density clumps at the base of the method. This will largely facilitate the discovery of potential new (sub)classes of variability. We also stress that improvements in the handling and removal of the instrumental artifacts in the CoRoT photometric time series, which occurred for a larger number of objects in the presented samples, will reveal other potential new types of variability not found here and will hopefully seriously improve the already very good performance of our algorithm.

Finally, even though the main aim of this work was the preparation for the analysis of the CoRoT database, we believe that the results found in the LMC dataset justify a comparative analysis of the OGLE Galactic Bulge data using the same approach.

Acknowledgements. The research leading to these results has received funding from the Spanish Ministerio de Educación y Ciencia through grant AYA2005- 
04286, from the European Research Council under the European Community's Seventh Framework Programme (FP7/2007-2013)/ERC grant agreement No. 227224 (PROSPERITY), from the Belgian PRODEX programme under grant PEA C90199 (CoRoT Mission Data Exploitation II), and from the research Council of Leuven University under grant GOA/2008/04. The authors made use of the Spanish Virtual Observatory supported from the Spanish MEC through grants AyA2005-04286, AyA2005-24102-E. This publication makes use of data products from the two Micron All Sky Survey, which is a joint project of the University of Massachusetts and the Infrared Processing and Analysis Center/California Institute of Technology, funded by the National Aeronautics and Space Administration and the National Science Foundation.

\section{References}

Aerts, C. 2000, A\&A, 361, 245

Aerts, C., Eyer, L., \& Kestens, E. 1998, A\&A, 337, 790

Aerts, C., De Cat, P., Peeters, E., et al. 1999, A\&A, 343, 872

Aerts, C., Cuypers, J., De Cat, P., et al. 2004, A\&A, 415, 1079

Aerts, C., Christensen-Dalsgaard, J., Cunha, M., \& Kurtz, D. W. 2008, Sol. Phys., 251, 3

Auvergne, M., Bodin, P., Boisnard, L., et al. 2009, A\&A, 506, 411

Bentley, J. L. 1980, Commun. ACM, 23, 214

Brett, D. R., West, R. G., \& Wheatley, P. J. 2004, MNRAS, 353, 369

Briquet, M., Aerts, C., Lüftinger, T., et al. 2004, A\&A, 413, 273

Buchler, J. R., Goupil, M. J., \& Hansen, C. J. 1997, A\&A, 321, 159

Buchler, J. R., Wood, P. R., Keller, S., \& Soszyński, I. 2005, ApJ, 631, L151

Cheeseman, P., Stutz, J., Self, M., et al. 1988, in Proceedings of the Seventh National Conference of Artificial Intelligence, 607

De Cat, P., Aerts, C., De Ridder, J., et al. 2000, A\&A, 355, 1015

De Cat, P., Eyer, L., Cuypers, J., et al. 2006, A\&A, 449, 281

Debosscher, J., Sarro, L. M., Aerts, C., et al. 2007, A\&A, 475, 1159

Debosscher, J., Sarro, L. M., López, M., et al. 2009, A\&A, 506, 519

Degroote, P., Aerts, C., Ollivier, M., et al. 2009, A\&A, 506, 471
Eyer, L., \& Blake, C. 2005, MNRAS, 358, 30

Eyer, L., \& Grenon, M. 1997, in Hipparcos - Venice '97, ESA SP, 402, 467 Freidman, J. H., Bentley, J. L., \& Finkel, R. A. 1977, ACM Trans. Math. Softw., 3, 209

Handler, G. 1999, MNRAS, 309, L19

Jain, A. K., Murty, M. N., \& Flynn, P. J. 1999, ACM Comput. Surv., 31, 264

Lefever, K., Puls, J., \& Aerts, C. 2007, A\&A, 463, 1093

Li, J., Ray, S., \& Lindsay, B. G. 2007, Journal of Machine Learning Research, 8, 1687

Mathias, P., Le Contel, J. M., Chapellier, E., et al. 2004, A\&A, 417, 189

Matthews, J. M. 2007, Commun. Asteroseismol., 150, 333

Meunier, J. C., Deleuil, M., Moutou, C., et al. 2007, in Astronomical Data Analysis Software and Systems XVI, ed. R. A. Shaw, F. Hill, \& D. J. Bell, ASP Conf. Ser., 376, 339

Perryman, M. A. C., \& ESA 1997, The HIPPARCOS and TYCHO catalogues, Astrometric and photometric star catalogues derived from the ESA HIPPARCOS Space Astrometry Mission (Noordwijk, Netherlands: ESA Publications Division), ESA SP Ser., 1200

Pojmanski, G. 2002, Acta Astron., 52, 397

Pollard, K. S., \& Laan, M. J. V. D. 2002, in Sixth World Multiconference on Systemics, Cybernetics, and Informatics, 318

Porter, J. M., \& Rivinius, T. 2003, PASP, 115, 1153

Rodriguez, E., \& López-González, M. J. 2000, Delta Scuti Star Newsletter, 14, 33

Samus, N. N., Goranskii, V. P., Durlevich, O. V., et al. 2002, VizieR Online Data Catalog, 902, 80201

Sarro, L. M., Debosscher, J., López, M., \& Aerts, C. 2009, A\&A, 358, 30

Skrutskie, M. F., Cutri, R. M., Stiening, R., et al. 2006, AJ, 131, 1163

Soszynski, I., Udalski, A., Kubiak, M., et al. 2004, Acta Astron., 54, 347

Soszynski, I., Udalski, A., Kubiak, M., et al. 2005, Acta Astron., 55, 331

Tibshirani, R., Walther, G., \& Hastie, T. 2000, Technical Report, 208, Dept. of Statistics, Stanford University, 63, 411

Waelkens, C., Aerts, C., Kestens, E., Grenon, M., \& Eyer, L. 1998, A\&A, 330, 215

Zebrun, K., Soszynski, I., Wozniak, P. R., et al. 2001, Acta Astron., 51, 317 


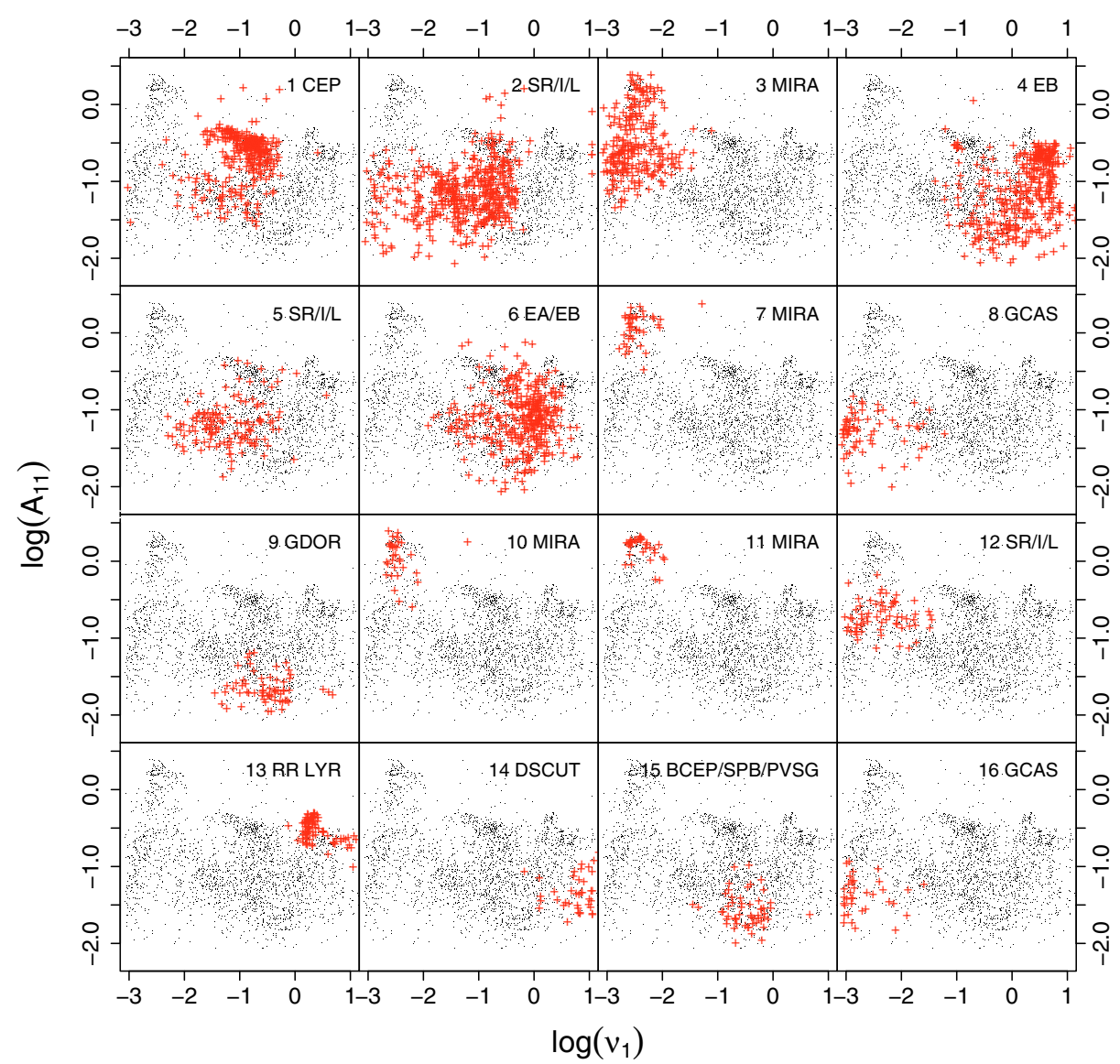

Fig. 18. The clustering structure of the Hipparcos archive at $\sigma=0.2$. The $x$-axis represents the logarithm of the frequency and the $y$-axis, the logarithm of the amplitude of the first component in the Fourier decomposition. Black dots represent the complete database and red crosses identify cluster members.

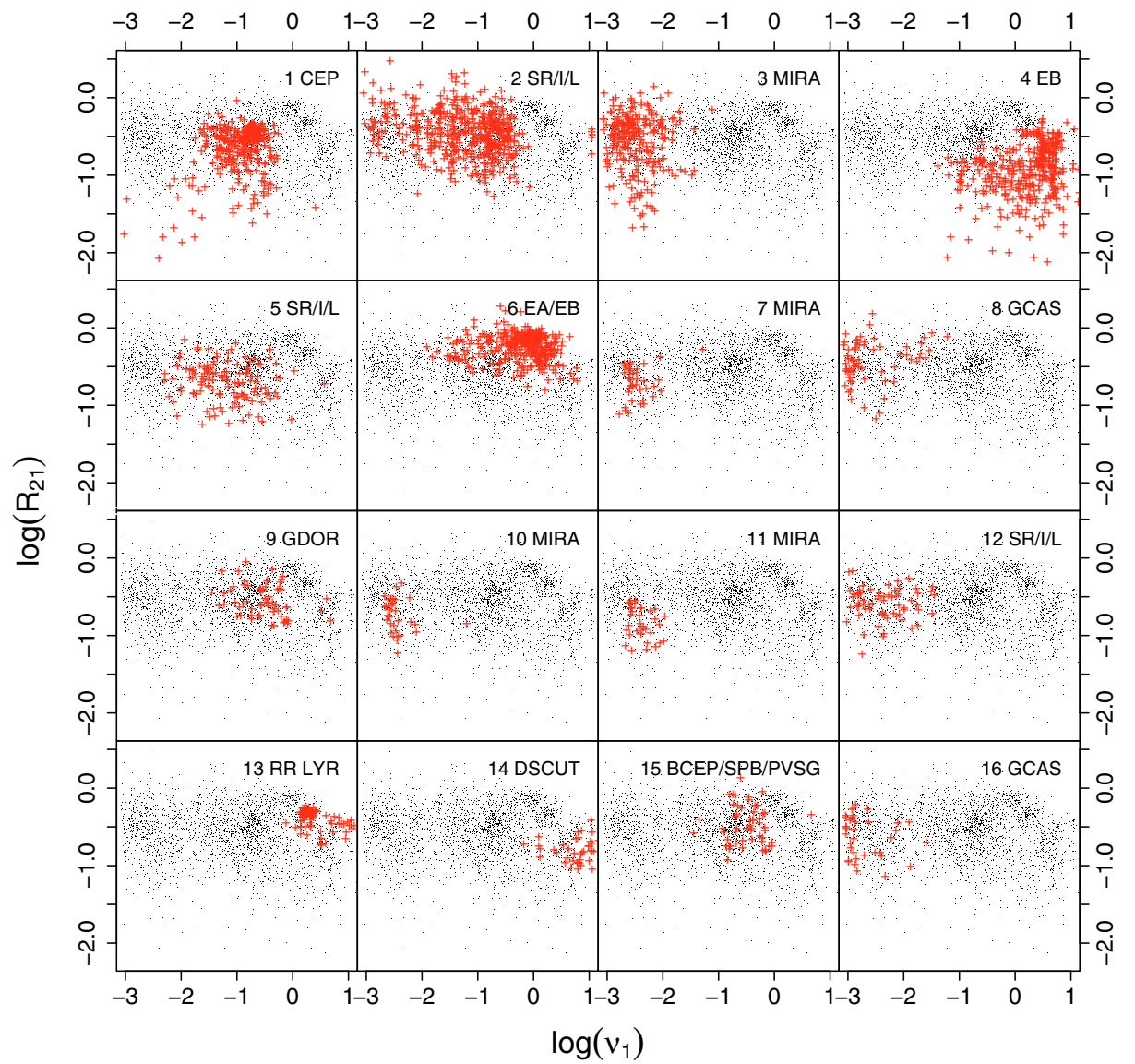

Fig. 19. The clustering structure of the Hipparcos archive at $\sigma=0.2$. The $x$-axis represents the logarithm of the frequency and the $y$-axis, the logarithm of the $R_{21}$ ratio between the amplitudes of the first two components in the Fourier decomposition. Black dots represent the complete database and red crosses identify cluster members. 


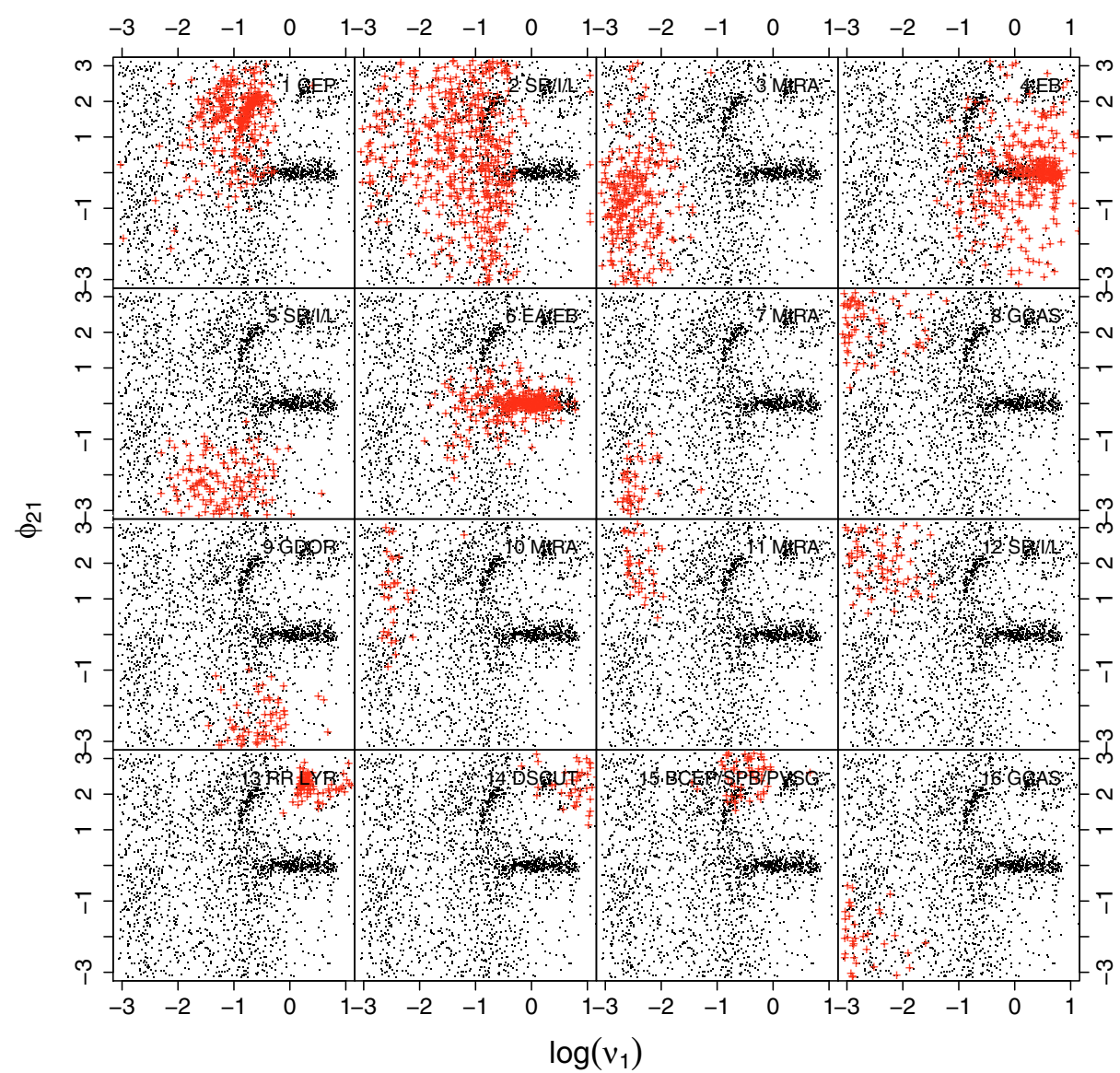

Fig. 20. The clustering structure of the Hipparcos archive at $\sigma=0.2$. The $x$-axis represents the logarithm of the frequency and the $y$-axis, the phase difference $\phi_{12}$ between the first two components in the Fourier decomposition. Black dots represent the complete database and red crosses identify cluster members.

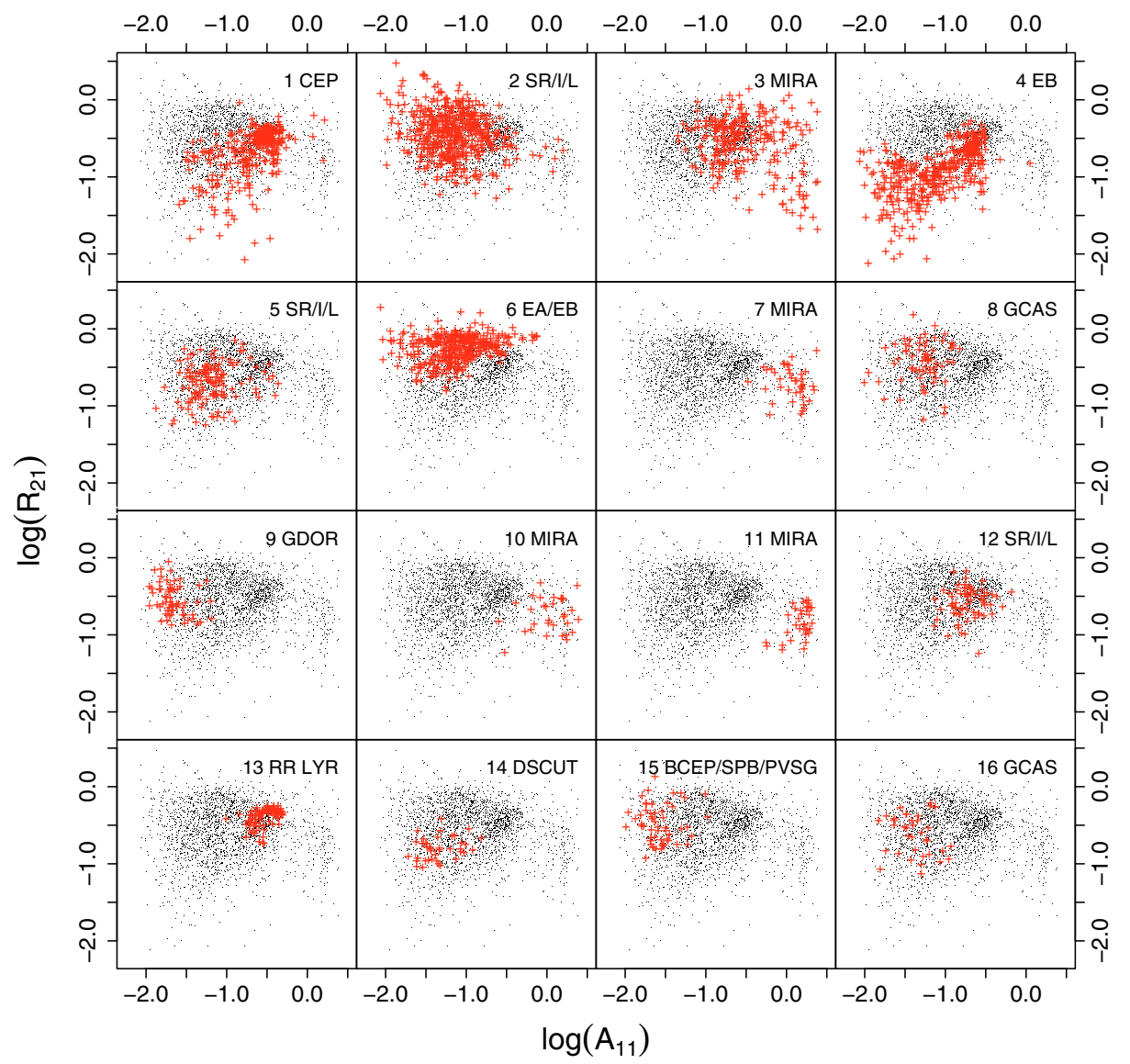

Fig. 21. The clustering structure of the Hipparcos archive at $\sigma=0.2$. The $x$-axis represents the logarithm of the amplitude of the first component in the Fourier decomposition, and the $y$-axis, the logarithm of the $R_{21}$ ratio between the amplitudes of the first two components. Black dots represent the complete database and red crosses identify cluster members. 


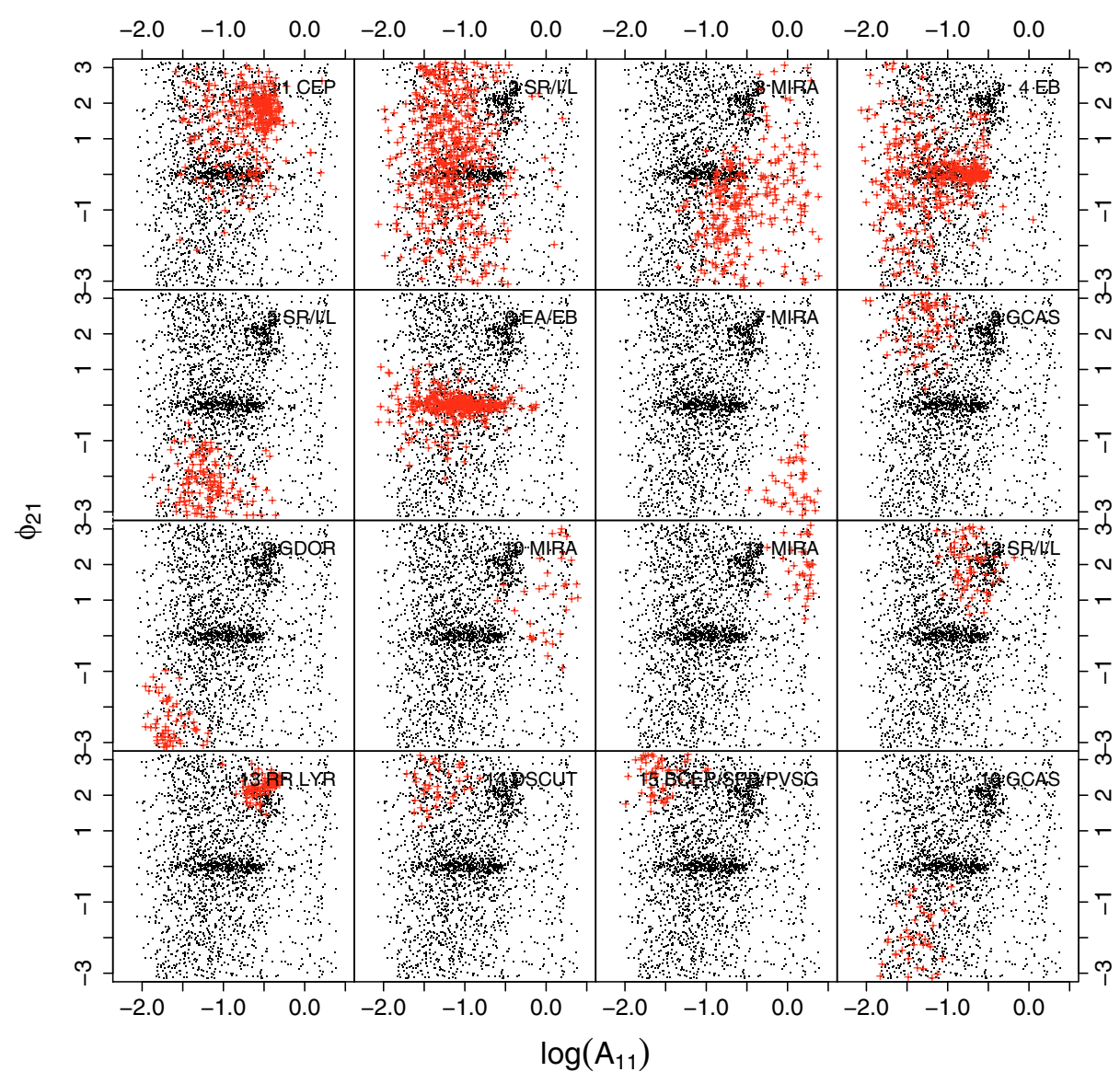

Fig. 22. The clustering structure of the Hipparcos archive at $\sigma=0.2$. The $x$-axis represents the logarithm of the amplitude of the first component in the Fourier decomposition, and the $y$-axis, the phase difference $\phi_{12}$ between the first two components in the Fourier decomposition. Black dots represent the complete database and red crosses identify cluster members.

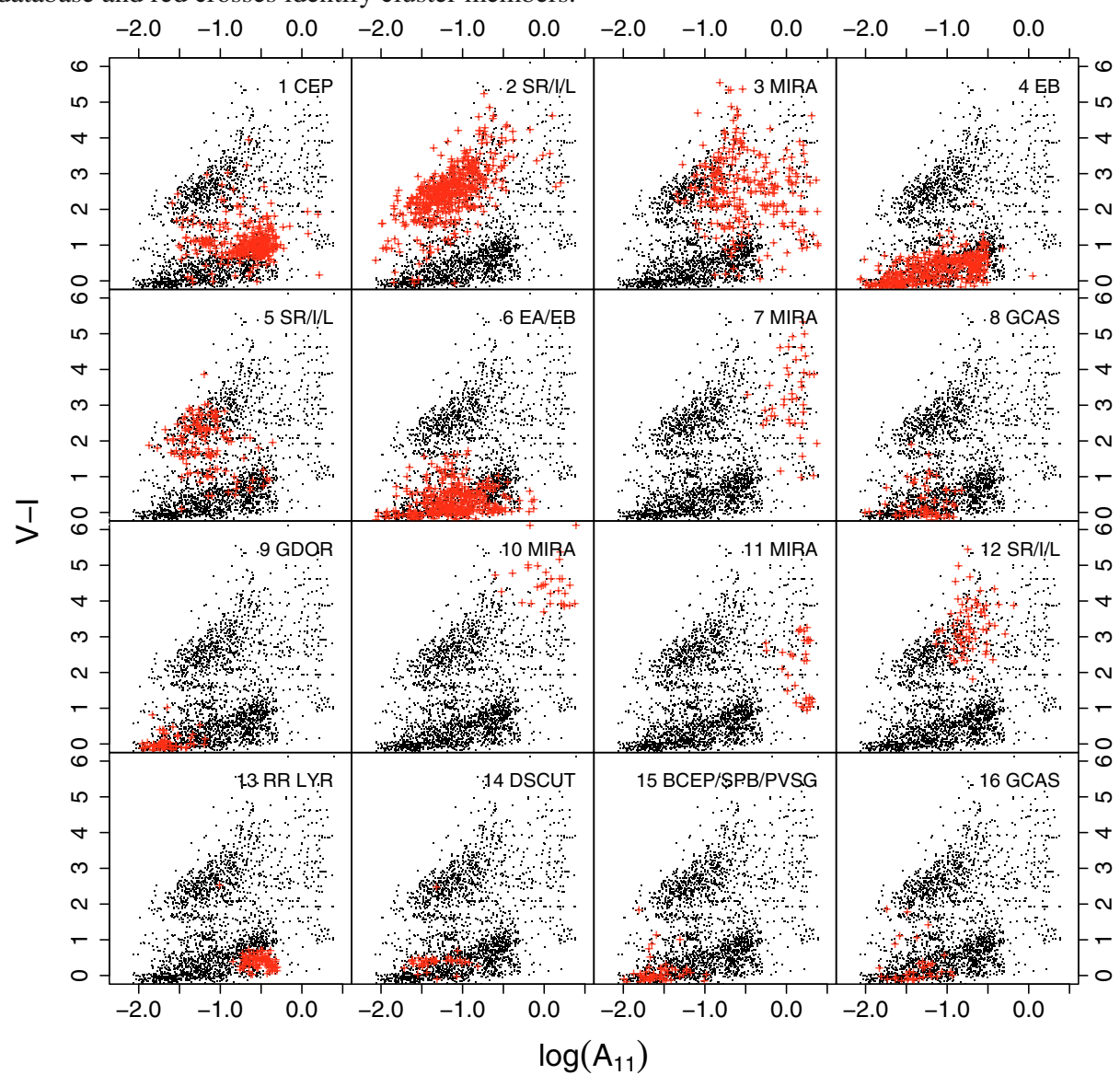

Fig. 23. The clustering structure of the Hipparcos archive at $\sigma=0.2$. The $x$-axis represents the logarithm of the amplitude of the first component in the Fourier decomposition, and the $y$-axis, the $V-I$ colour index. Black dots represent the complete database and red crosses identify cluster members. 


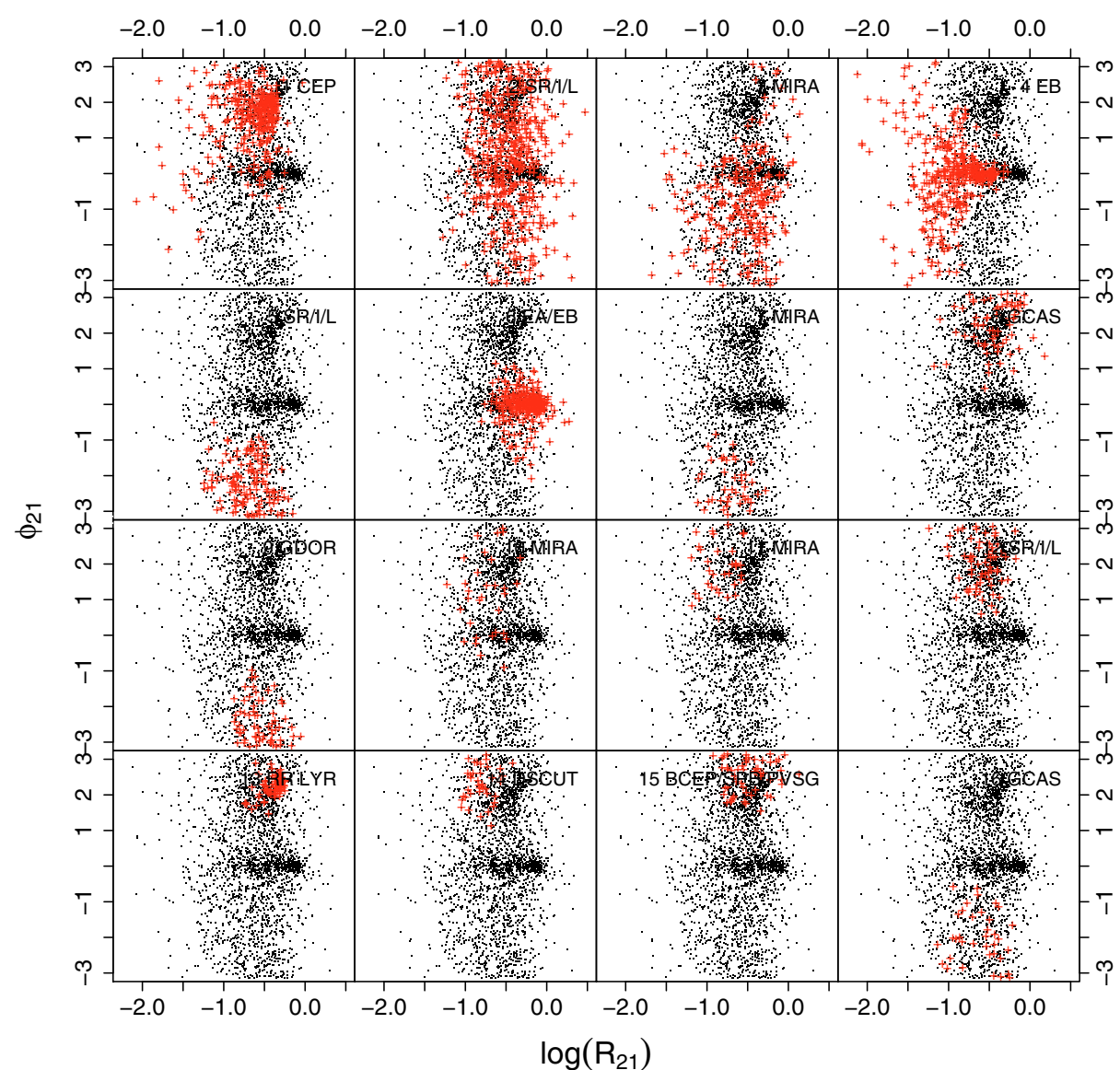

Fig. 24. The clustering structure of the Hipparcos archive at $\sigma=0.2$. The $x$-axis represents the logarithm of the $R_{21}$ ratio between the amplitudes of the first two components of the Fourier decomposition, and the $y$-axis, the phase difference $\phi_{12}$ between the first two components in the Fourier decomposition. Black dots represent the complete database and red crosses identify cluster members.

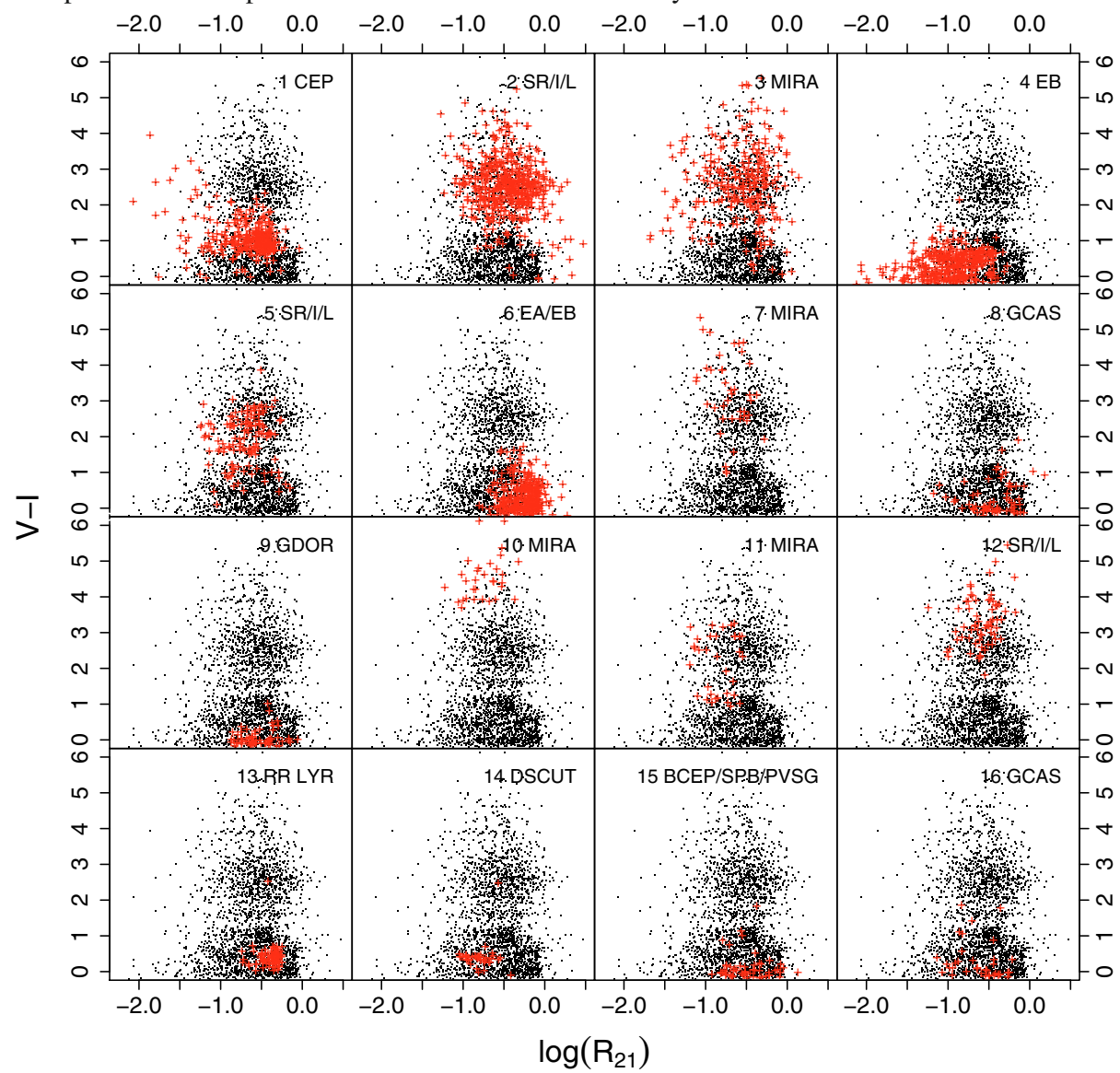

Fig. 25. The clustering structure of the Hipparcos archive at $\sigma=0.2$. The $x$-axis represents the logarithm of the $R_{21}$ ratio between the amplitudes of the first two components of the Fourier decomposition, and the $y$-axis, the $V-I$ colour index. Black dots represent the complete database and red crosses identify cluster members. 


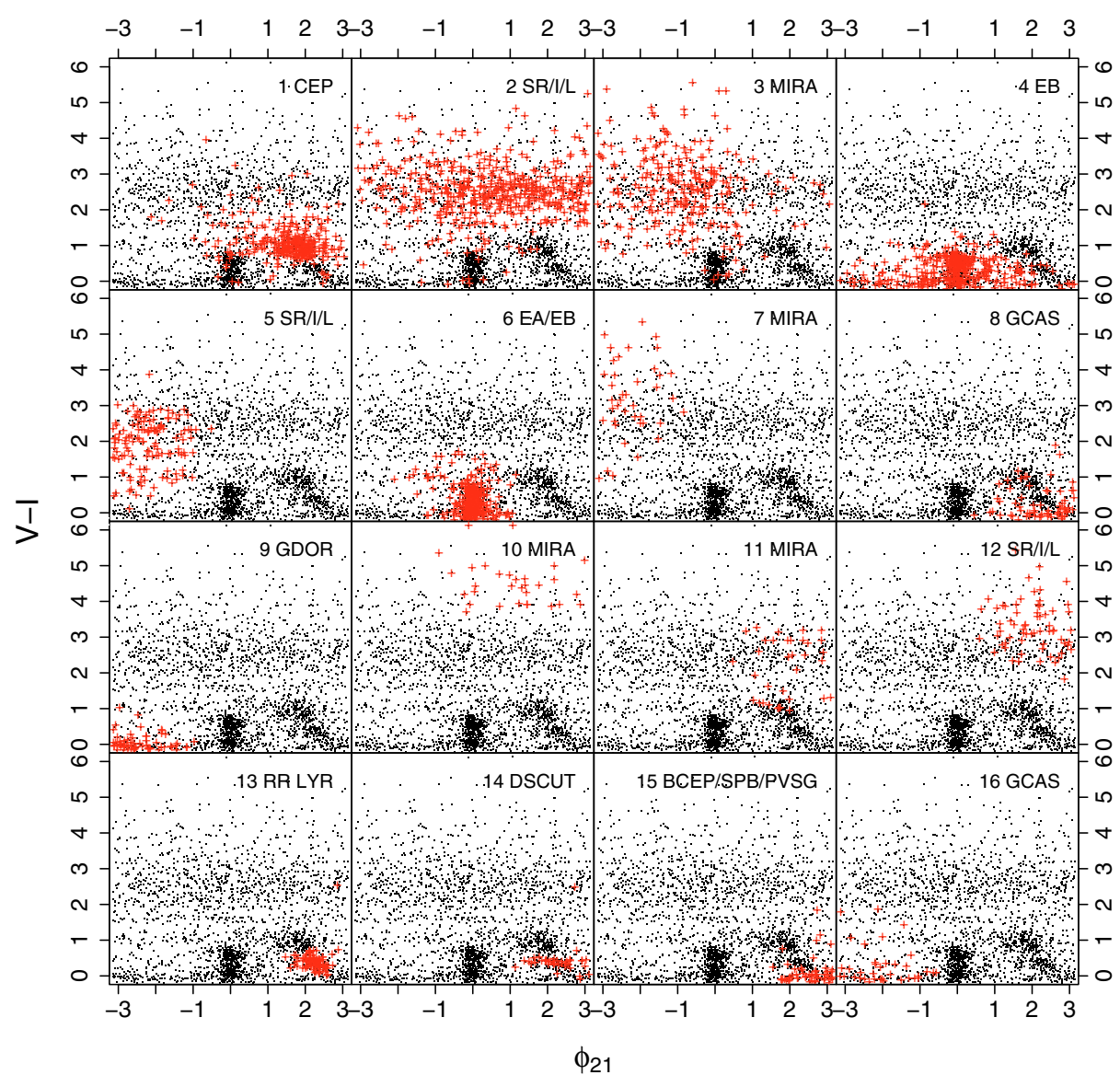

Fig. 26. The clustering structure of the Hipparcos archive at $\sigma=0.2$. The $x$-axis represents the phase difference $\phi_{12}$ between the first two components in the Fourier decomposition, and the $y$-axis, the $V-I$ colour index. Black dots represent the complete database and red crosses identify cluster members.

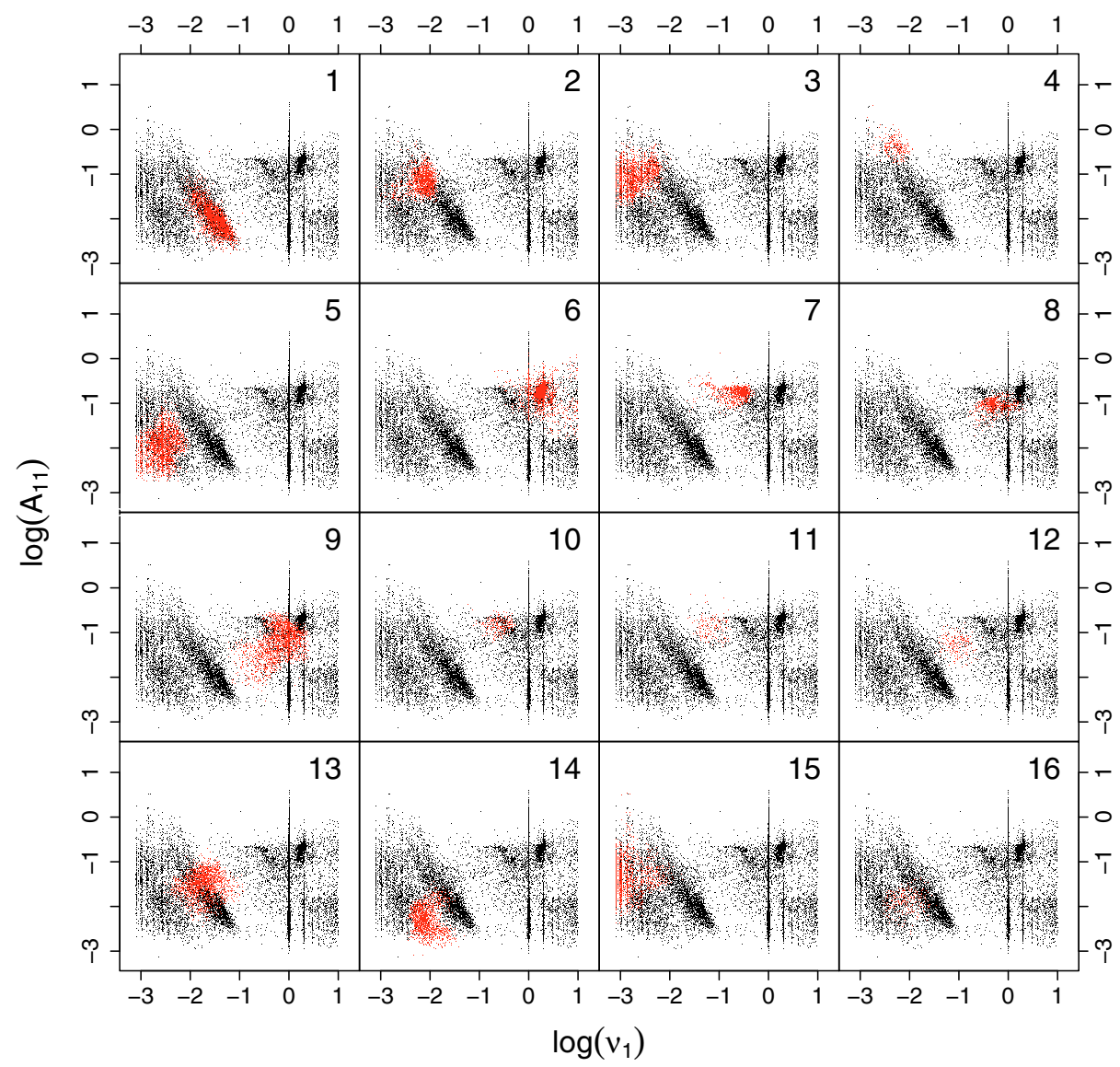

Fig. 27. The clustering structure of the OGLE LMC archive at $\sigma=0.15$. The $x$-axis represents the logarithm of the frequency and the $y$-axis, the logarithm of the amplitude of the first component in the Fourier decomposition. Black dots represent the complete database and red crosses identify cluster members. 


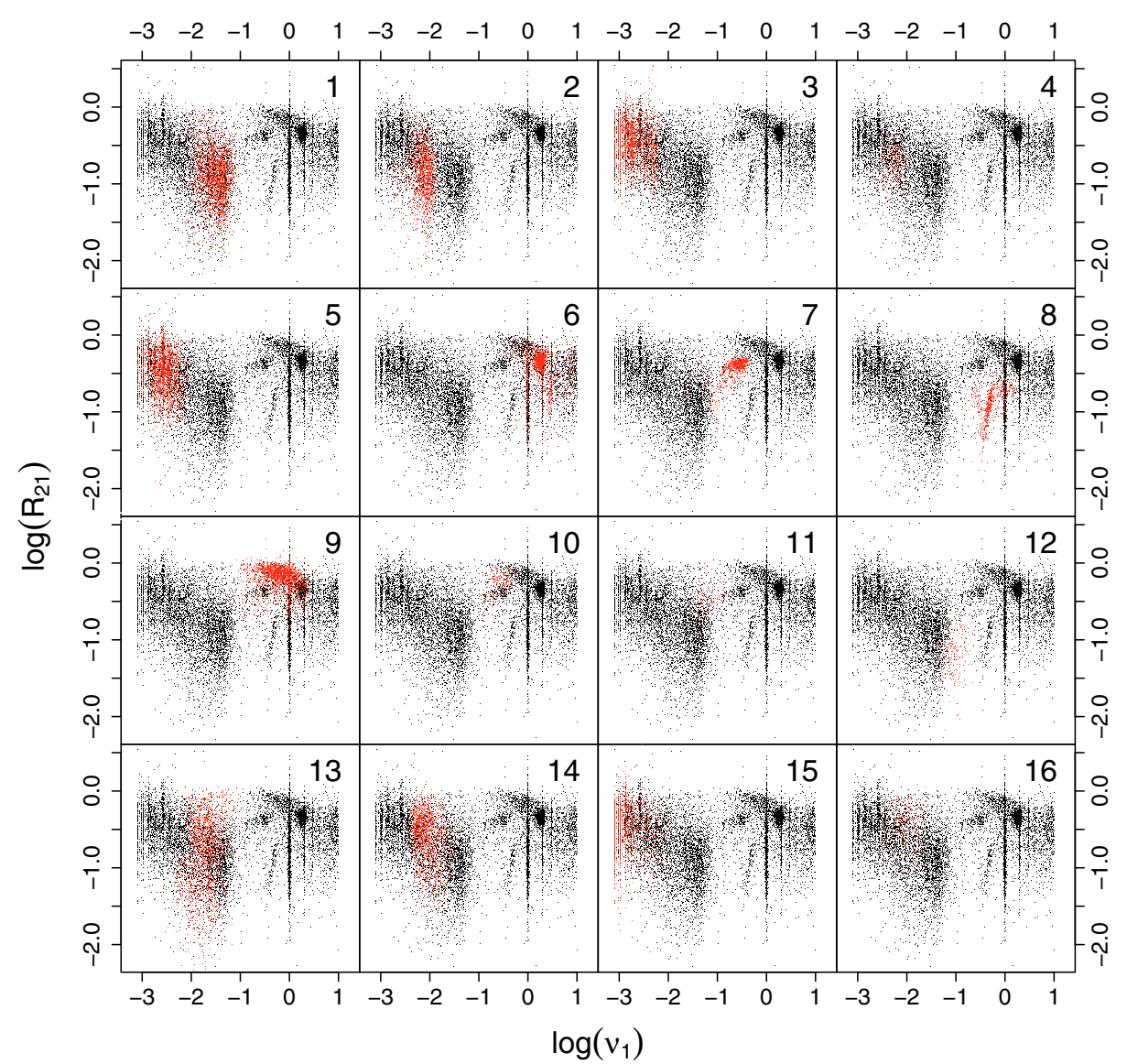

Fig. 28. The clustering structure of the OGLE LMC archive at $\sigma=0.15$. The $x$-axis represents the logarithm of the frequency and the $y$-axis, the logarithm of the $R_{21}$ ratio between the amplitudes of the first two components in the Fourier decomposition. Black dots represent the complete database and red crosses identify cluster members.

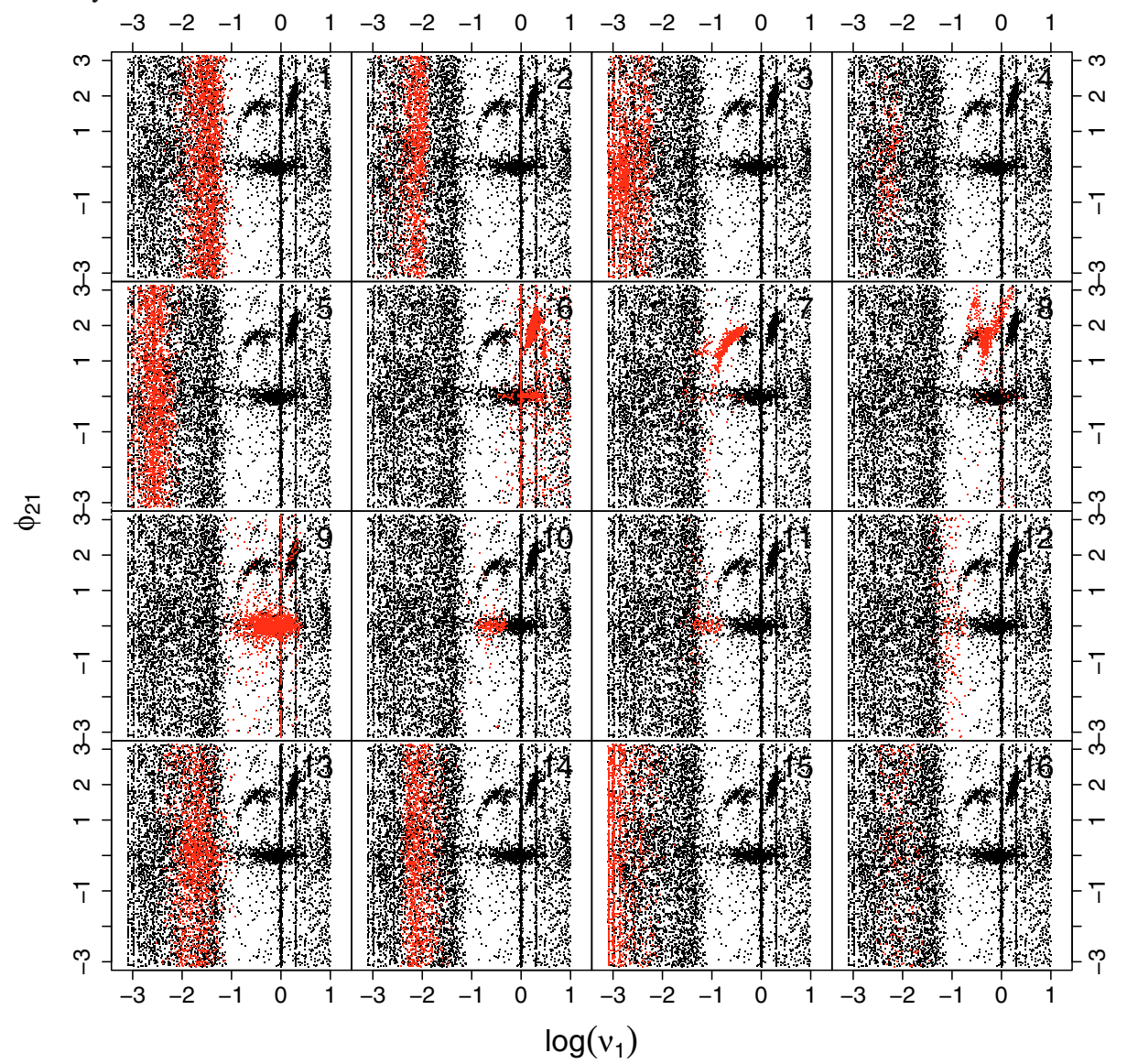

Fig. 29. The clustering structure of the OGLE LMC archive at $\sigma=0.15$. The $x$-axis represents the logarithm of the frequency and the $y$-axis, the phase difference $\phi_{12}$ between the first two components in the Fourier decomposition. Black dots represent the complete database and red crosses identify cluster members. 


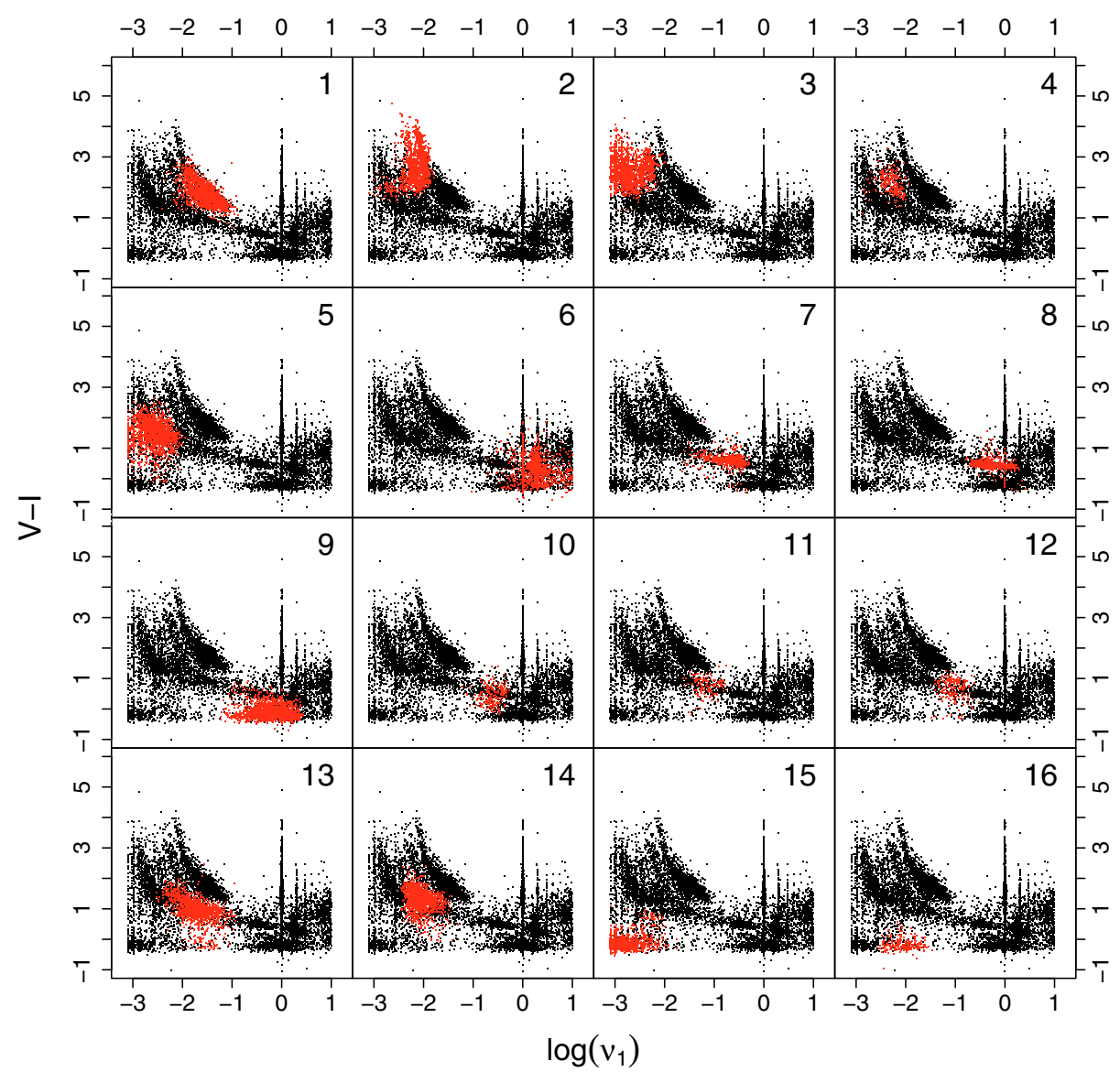

Fig. 30. The clustering structure of the OGLE LMC archive at $\sigma=0.15$. The $x$-axis represents the logarithm of the frequency and the $y$-axis, the $V-I$ colour index. Black dots represent the complete database and red crosses identify cluster members.

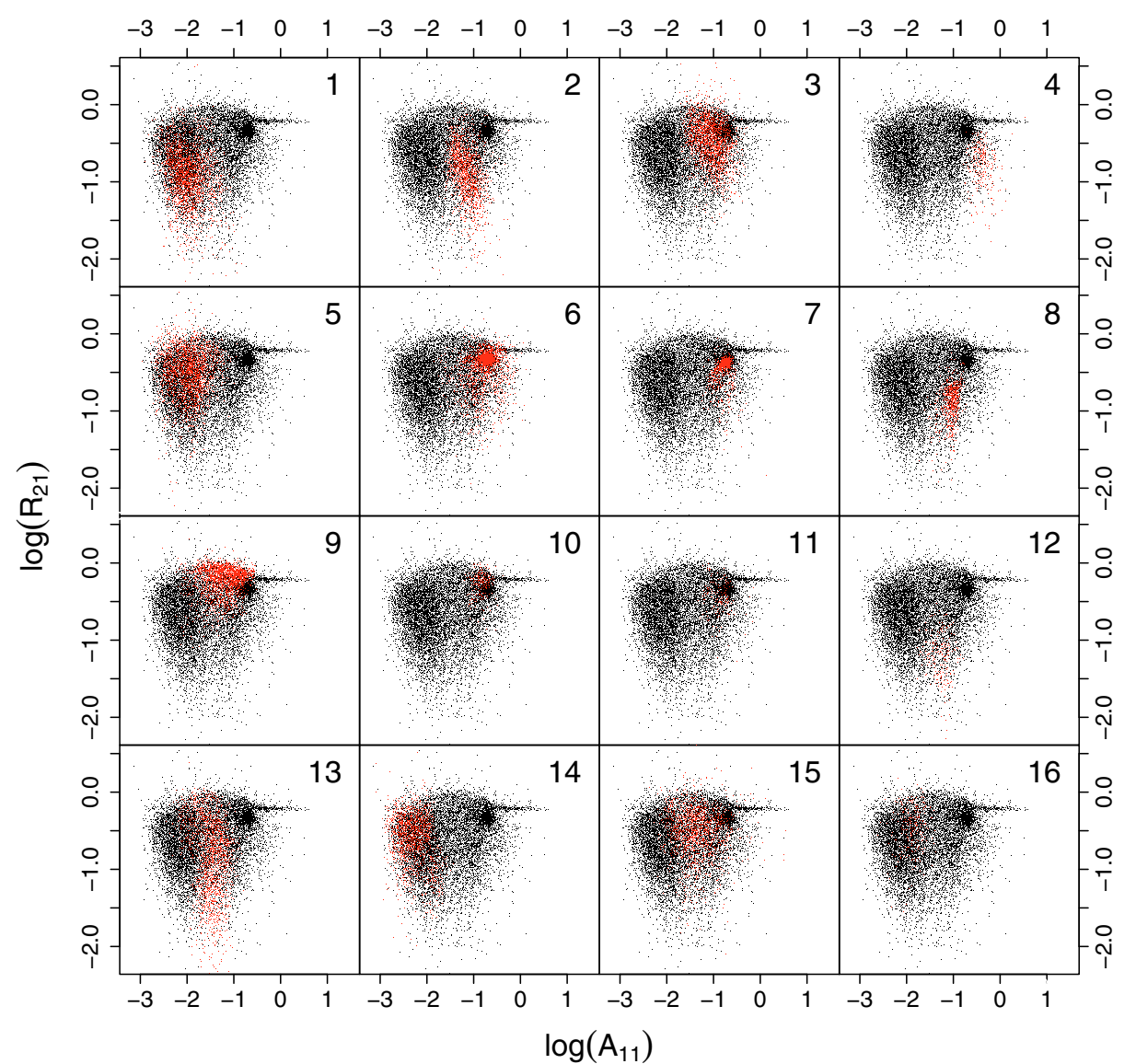

Fig. 31. The clustering structure of the OGLE LMC archive at $\sigma=0.15$. The $x$-axis represents the logarithm of the amplitude of the first component in the Fourier decomposition, and the $y$-axis, the logarithm of the $R_{21}$ ratio between the amplitudes of the first two components. Black dots represent the complete database and red crosses identify cluster members. 


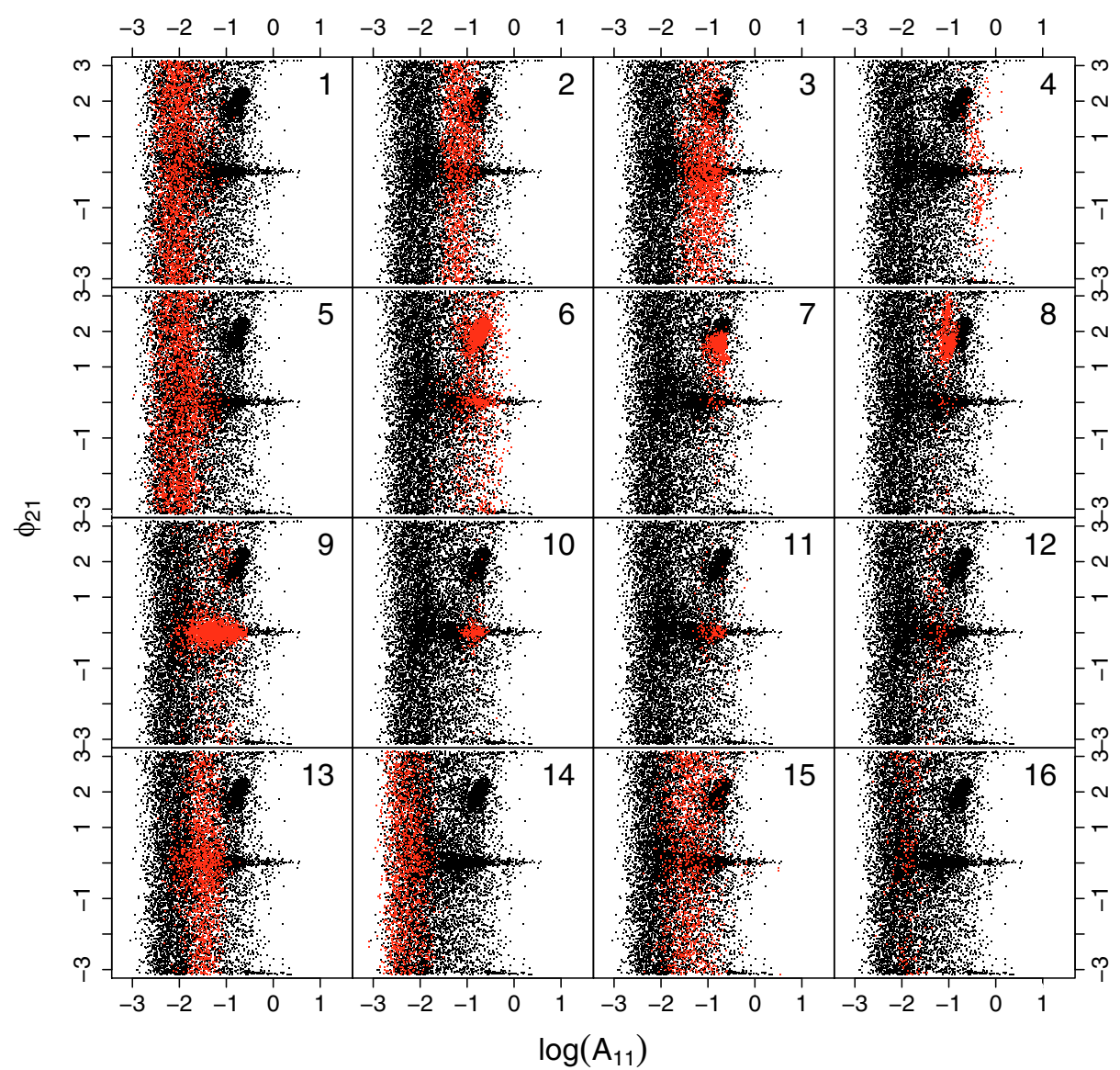

Fig. 32. The clustering structure of the OGLE LMC archive at $\sigma=0.15$. The $x$-axis represents the logarithm of the amplitude of the first component in the Fourier decomposition, and the $y$-axis, the phase difference $\phi_{12}$ between the first two components in the Fourier decomposition. Black dots represent the complete database and red crosses identify cluster members.

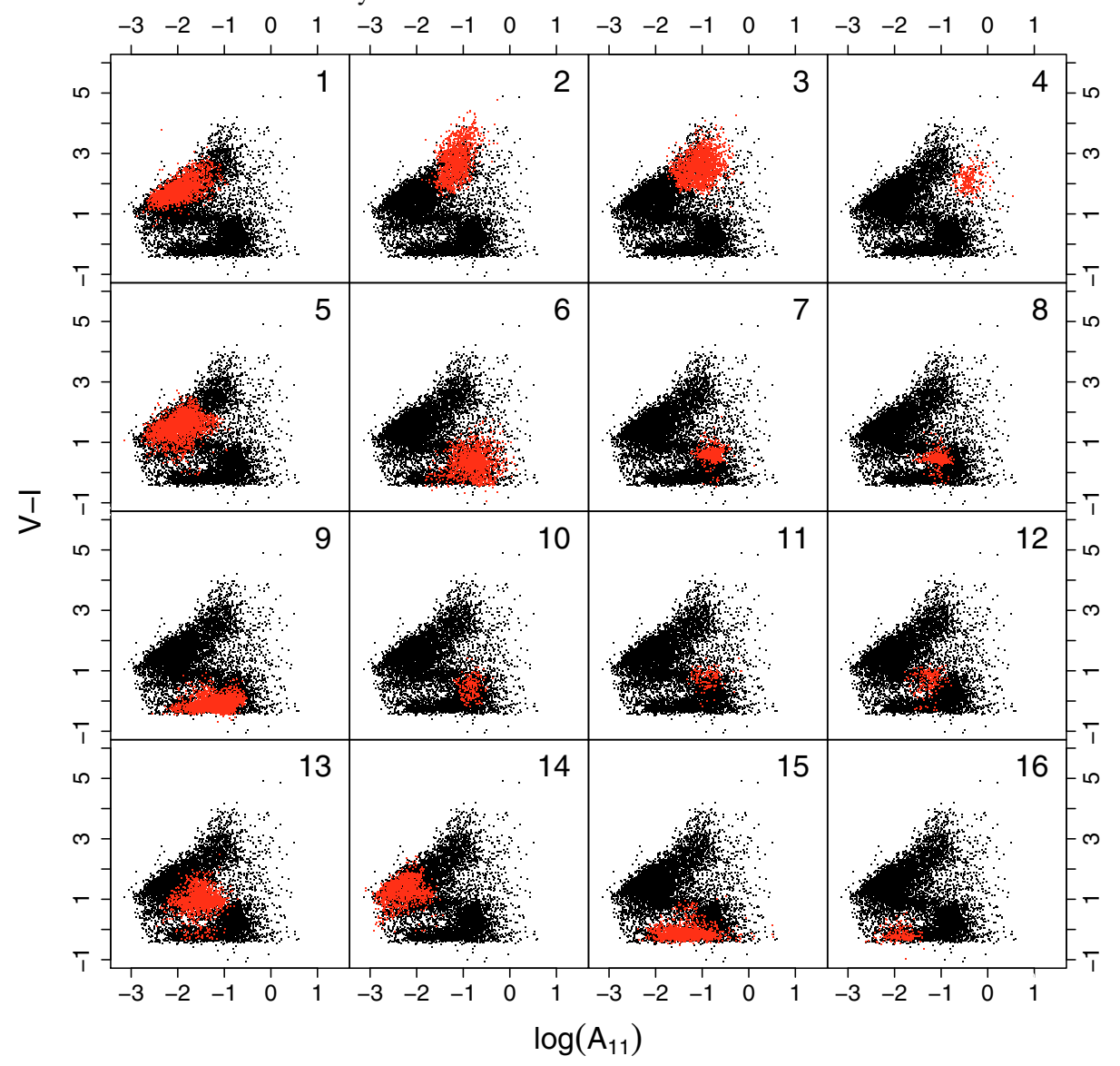

Fig. 33. The clustering structure of the OGLE LMC archive at $\sigma=0.15$. The $x$-axis represents the logarithm of the amplitude of the first component in the Fourier decomposition, and the $y$-axis, the $V-I$ colour index. Black dots represent the complete database and red crosses identify cluster members. 


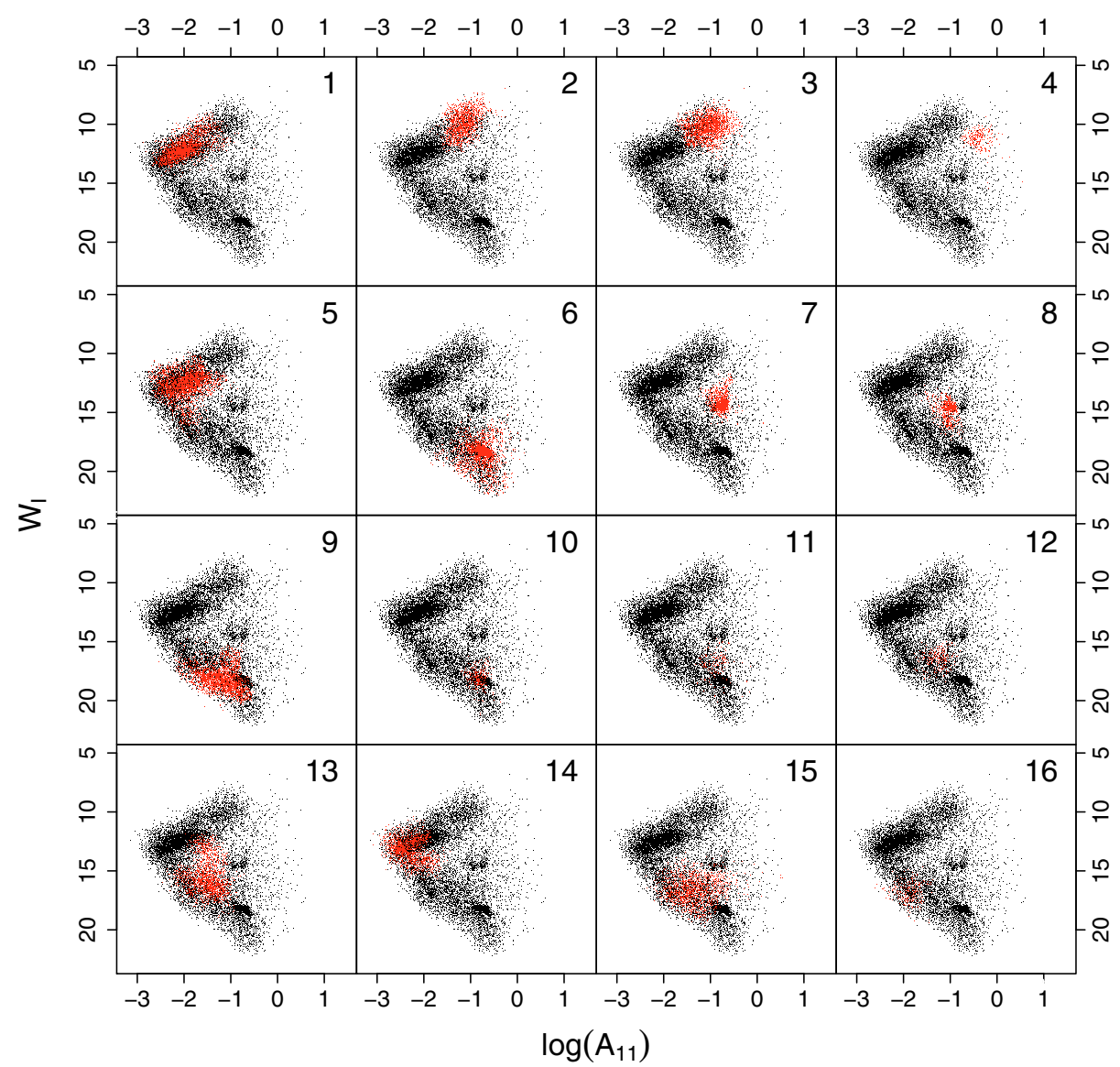

Fig. 34. The clustering structure of the OGLE LMC archive at $\sigma=0.15$. The $x$-axis represents the logarithm of the amplitude of the first component in the Fourier decomposition, and the $y$-axis, the Wessenheit index $W_{I}$. Black dots represent the complete database and red crosses identify cluster members.

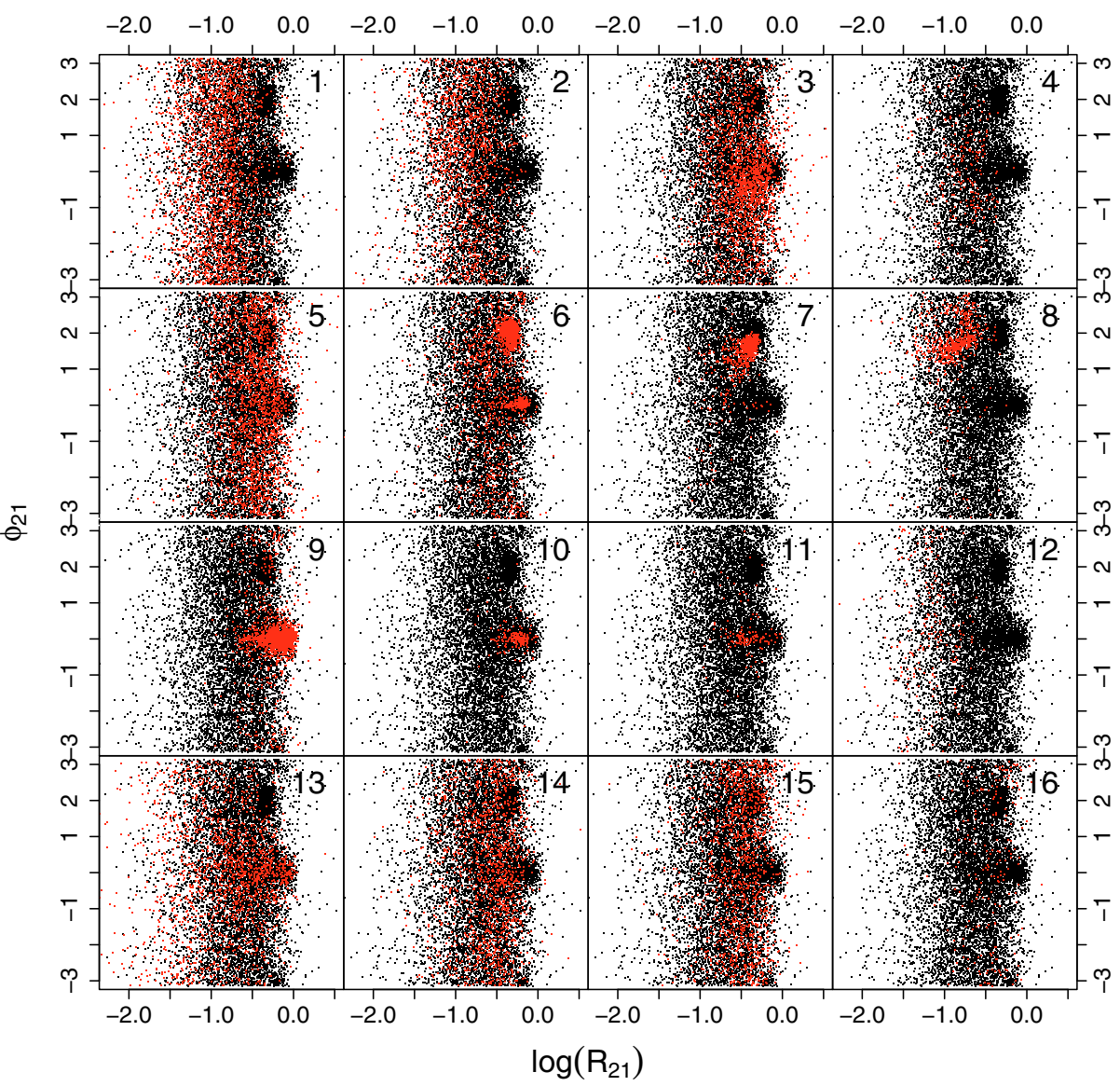

Fig. 35. The clustering structure of the OGLE LMC archive at $\sigma=0.15$. The $x$-axis represents the logarithm of the $R_{21}$ ratio between the amplitudes of the first two components of the Fourier decomposition, and the $y$-axis, the phase difference $\phi_{12}$ between the first two components in the Fourier decomposition. Black dots represent the complete database and red crosses identify cluster members. 


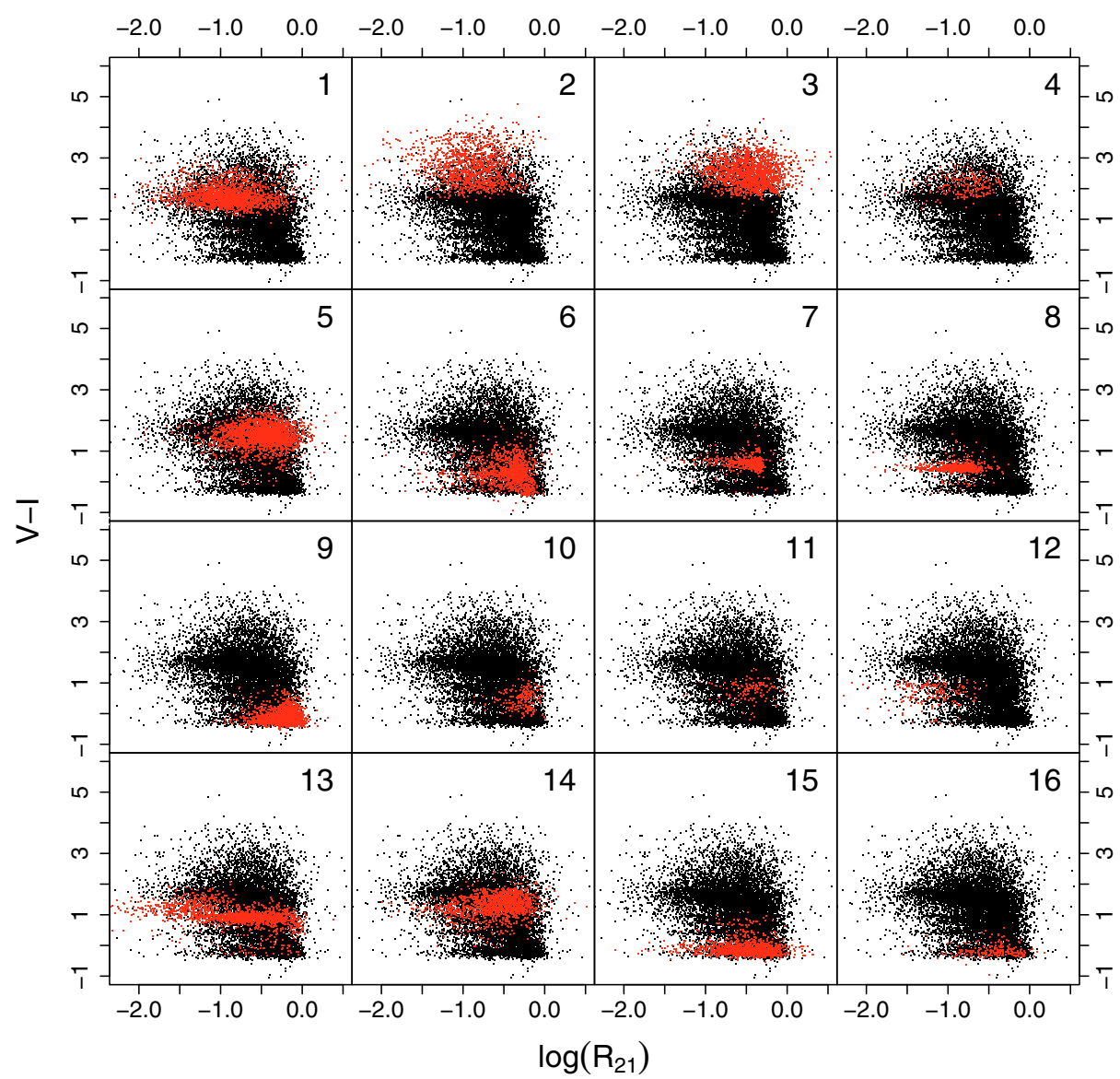

Fig. 36. The clustering structure of the OGLE LMC archive at $\sigma=0.15$. The $x$-axis represents the logarithm of the $R_{21}$ ratio between the amplitudes of the first two components of the Fourier decomposition, and the $y$-axis, the $V-I$ colour index. Black dots represent the complete database and red crosses identify cluster members.

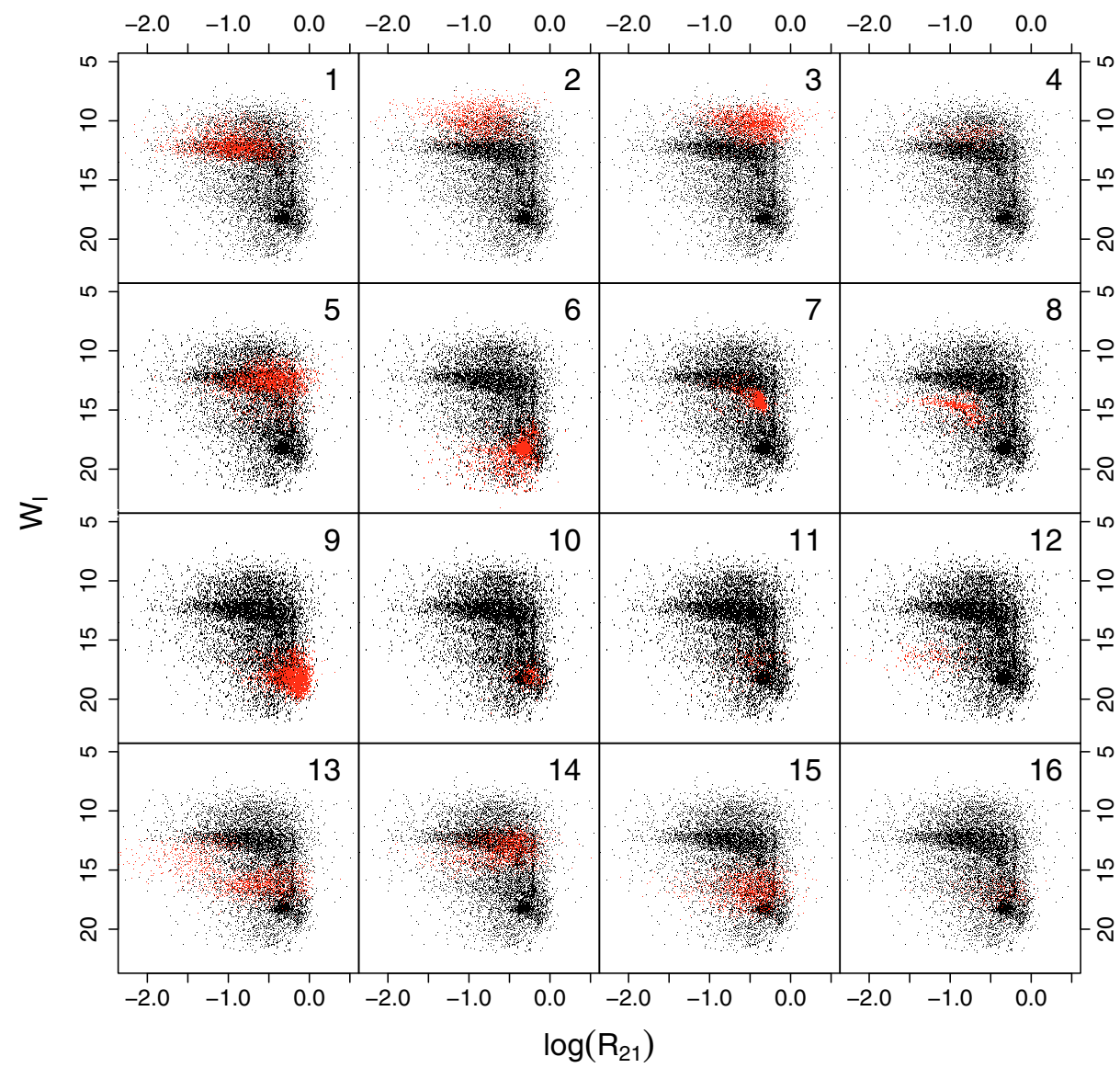

Fig. 37. The clustering structure of the OGLE LMC archive at $\sigma=0.15$. The $x$-axis represents the logarithm of the $R_{21}$ ratio between the amplitudes of the first two components of the Fourier decomposition, and the $y$-axis, the Wessenheit index $W_{I}$. Black dots represent the complete database and red crosses identify cluster members. 


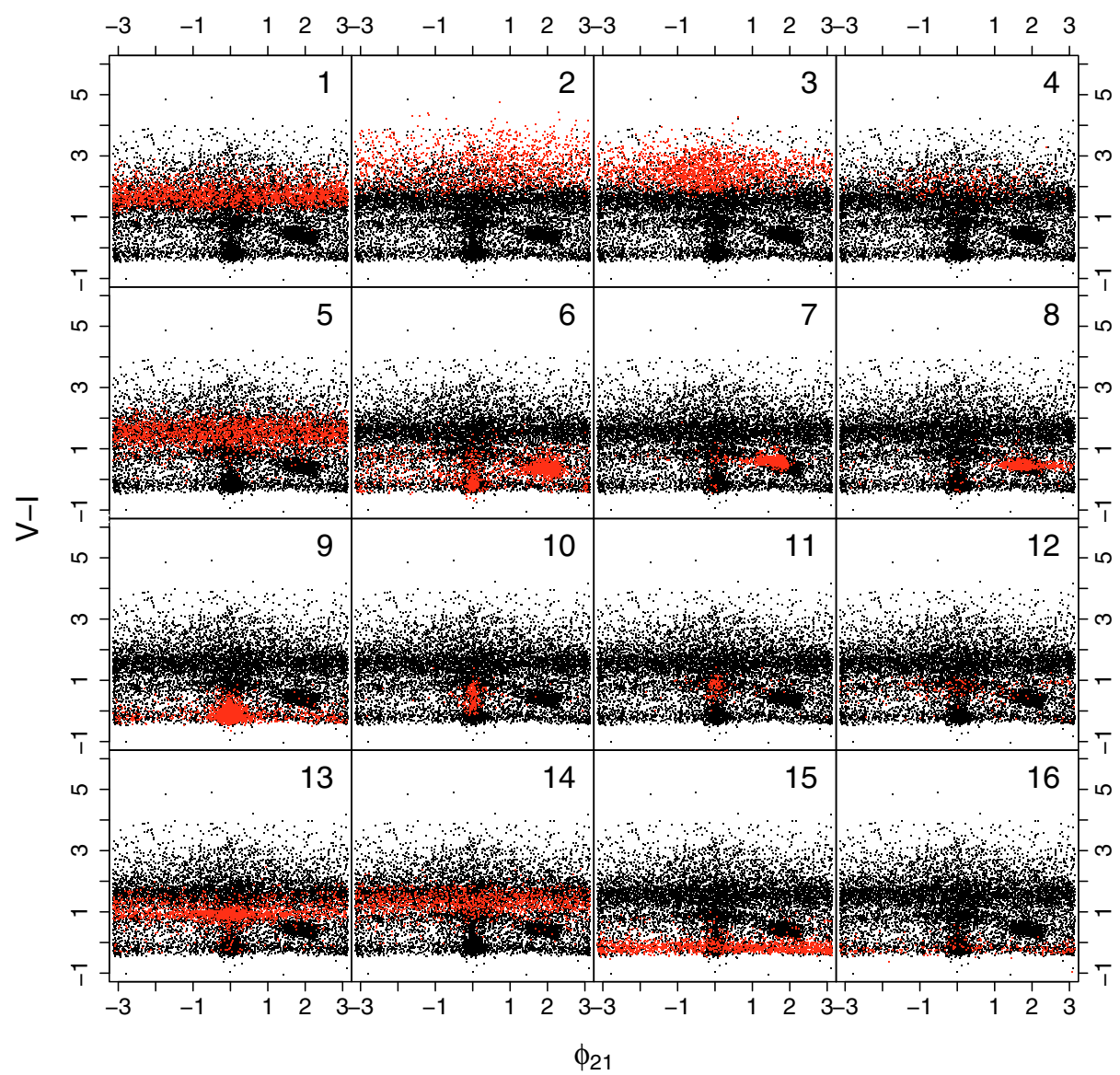

Fig. 38. The clustering structure of the OGLE LMC archive at $\sigma=0.15$. The $x$-axis represents the phase difference $\phi_{12}$ between the first two components in the Fourier decomposition, and the $y$-axis, the $V-I$ colour index. Black dots represent the complete database and red crosses identify cluster members.

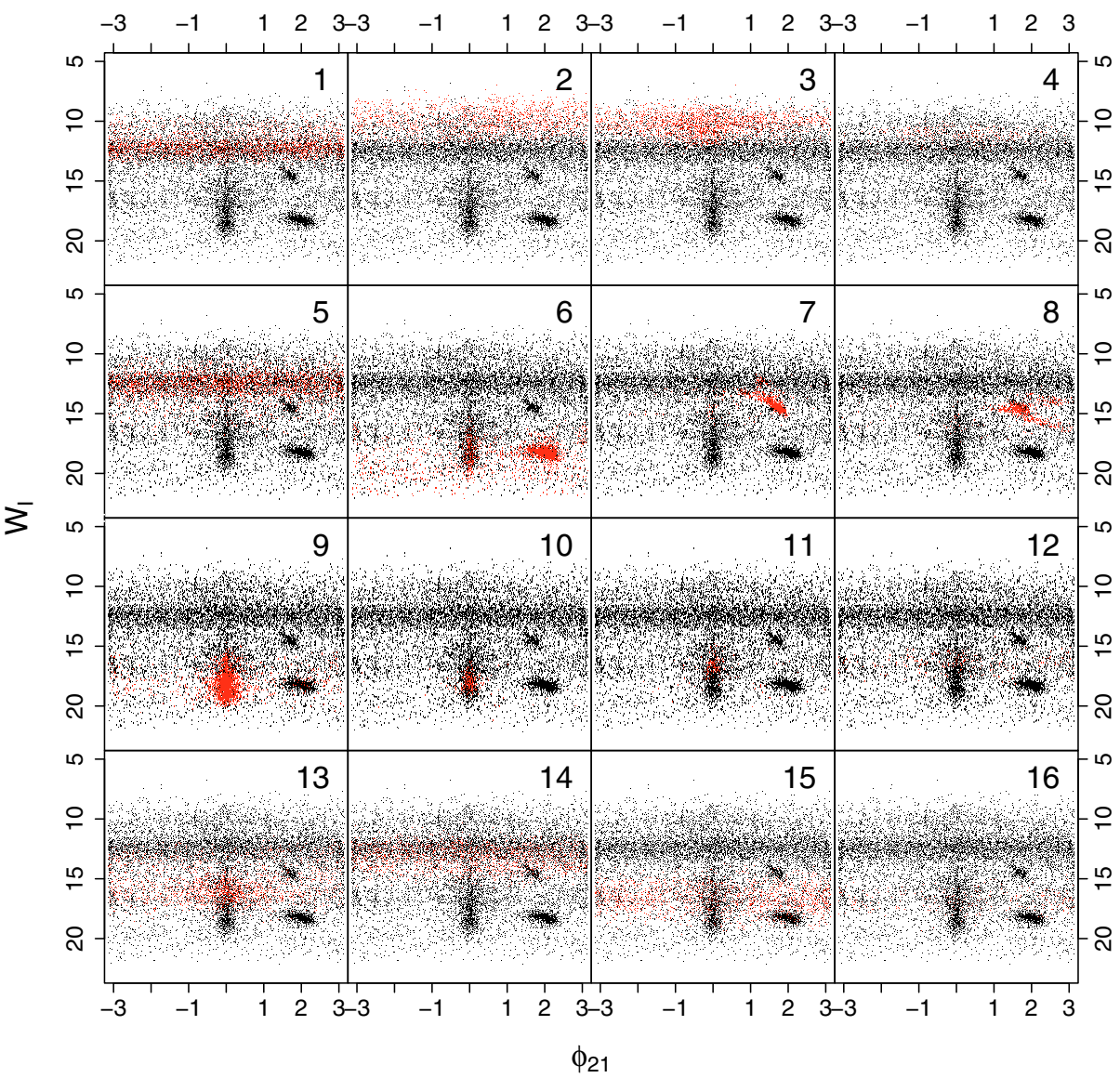

Fig. 39. The clustering structure of the OGLE LMC archive at $\sigma=0.15$. The $x$-axis represents the phase difference $\phi_{12}$ between the first two components in the Fourier decomposition, and the $y$-axis, the Wessenheit index $W_{I}$. Black dots represent the complete database and red crosses identify cluster members. 


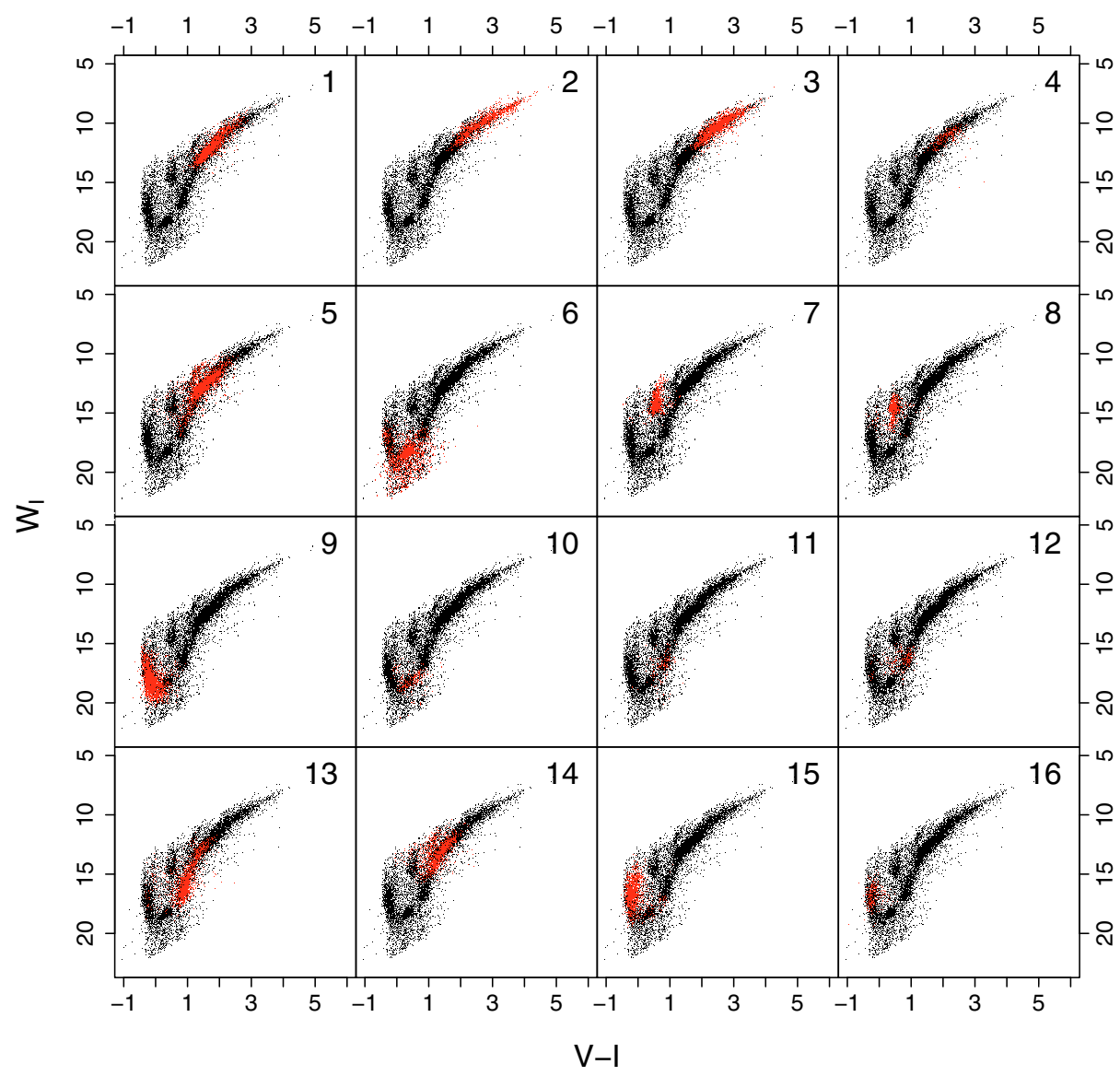

Fig. 40. The clustering structure of the OGLE LMC archive at $\sigma=0.15$. The $x$-axis represents the $V-I$ colour index, and the $y$-axis, the Wessenheit index $W_{I}$. Black dots represent the complete database and red crosses identify cluster members.

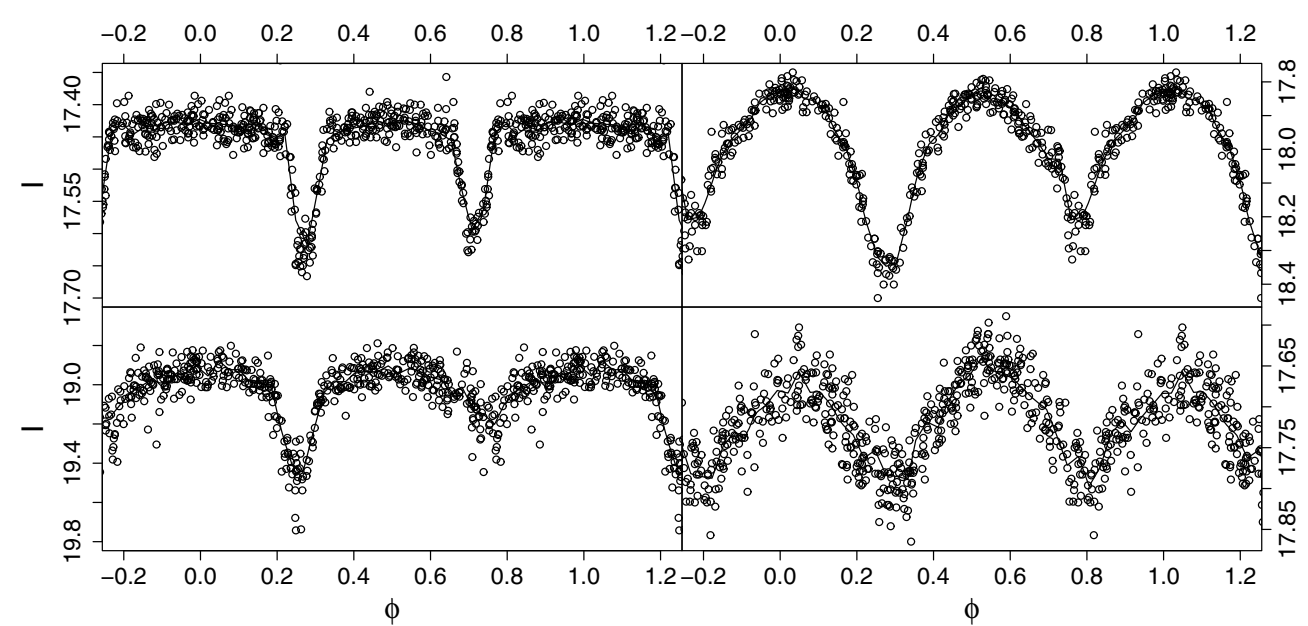

Fig. 41. Example light curves from objects in clusters 9 (top left), 10 (bottom left), 11 (top right), and 12 (bottom right), of the OGLE LMC sample. 


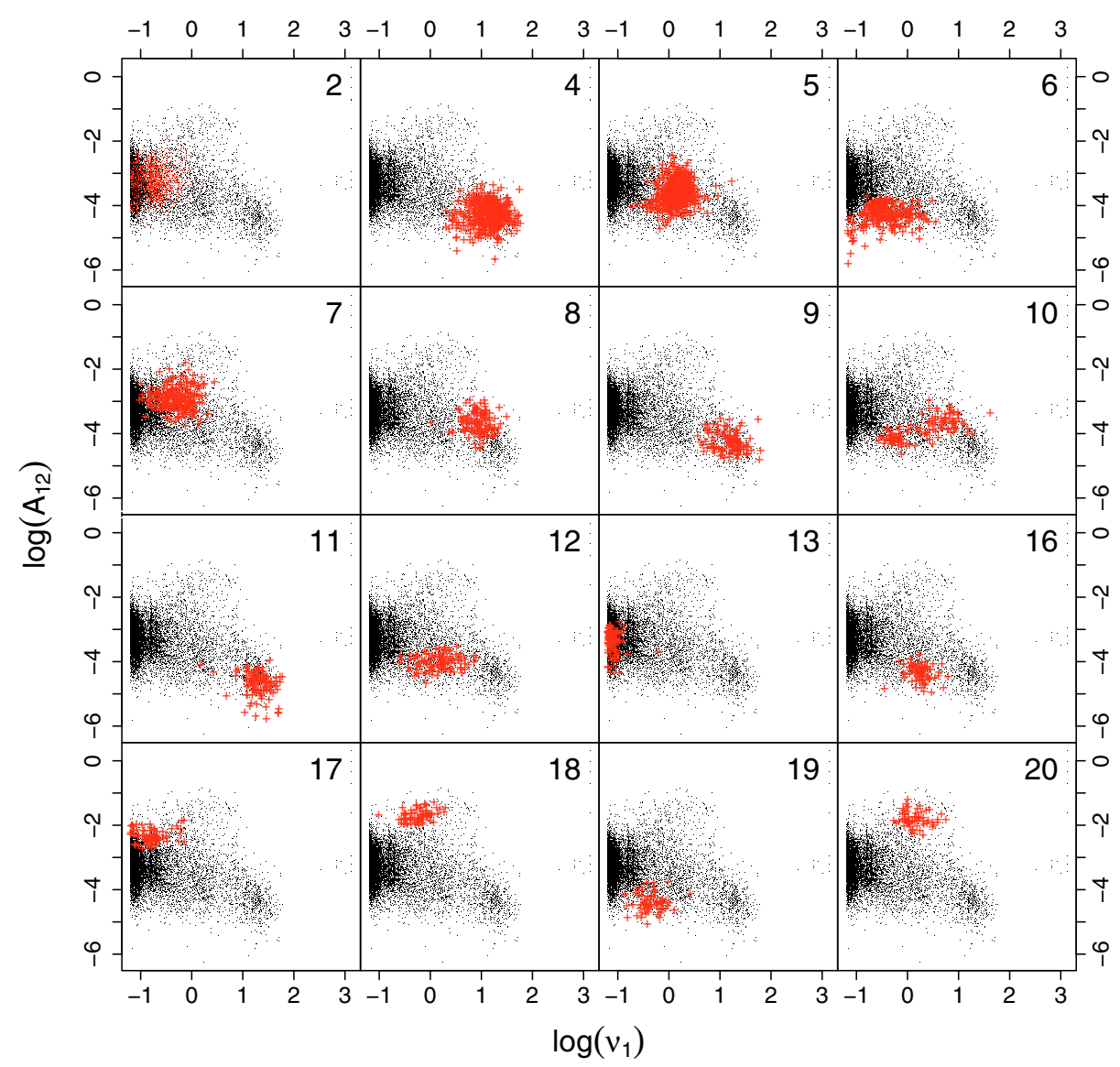

Fig. 42. The clustering structure of the CoRoT first four runs archive at $\sigma=0.2$. The $x$-axis represents the logarithm of the first frequency and the $y$-axis, the logarithm of the amplitude of the second Fourier component of the first detected frequency. Black dots represent the complete database and red crosses identify cluster members.

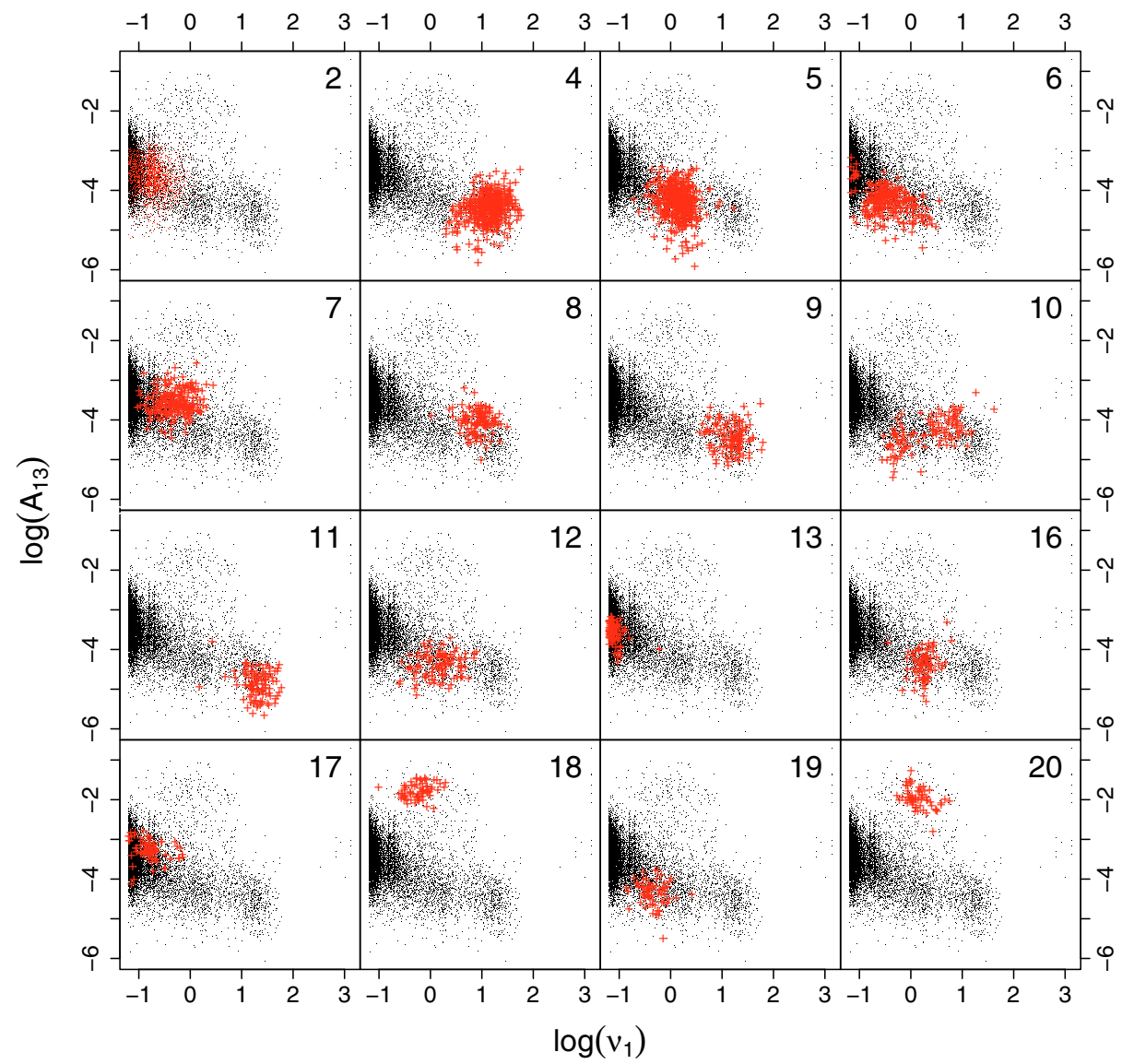

Fig. 43. The clustering structure of the CoRoT first four runs archive at $\sigma=0.2$. The $x$-axis represents the logarithm of the first frequency and the $y$-axis, the logarithm of the amplitude of the third Fourier component of the first detected frequency. Black dots represent the complete database and red crosses identify cluster members. 


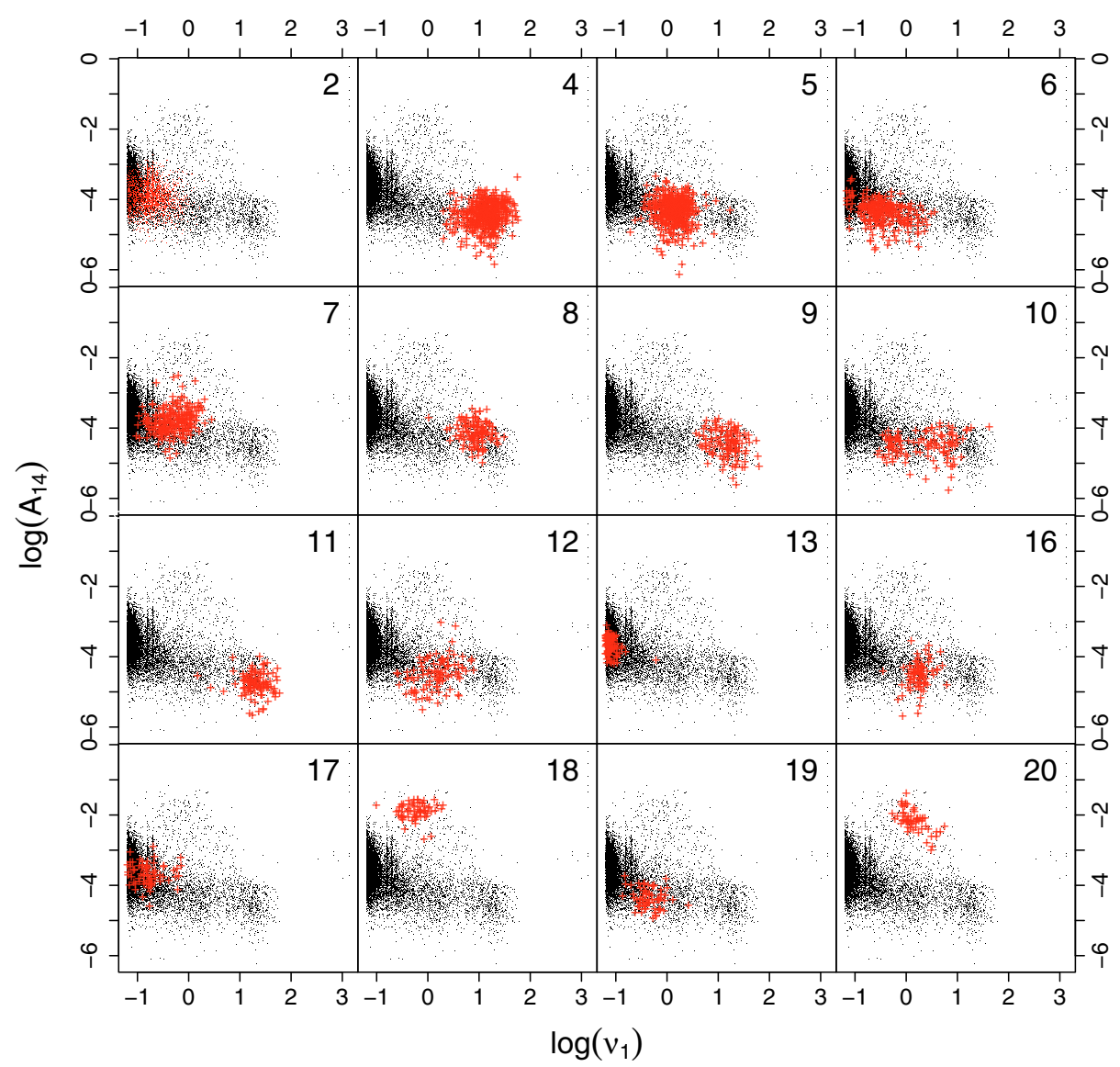

Fig. 44. The clustering structure of the CoRoT first four runs archive at $\sigma=0.2$. The $x$-axis represents the logarithm of the first frequency and the $y$-axis, the logarithm of the amplitude of the fourth Fourier component of the first detected frequency. Black dots represent the complete database and red crosses identify cluster members.

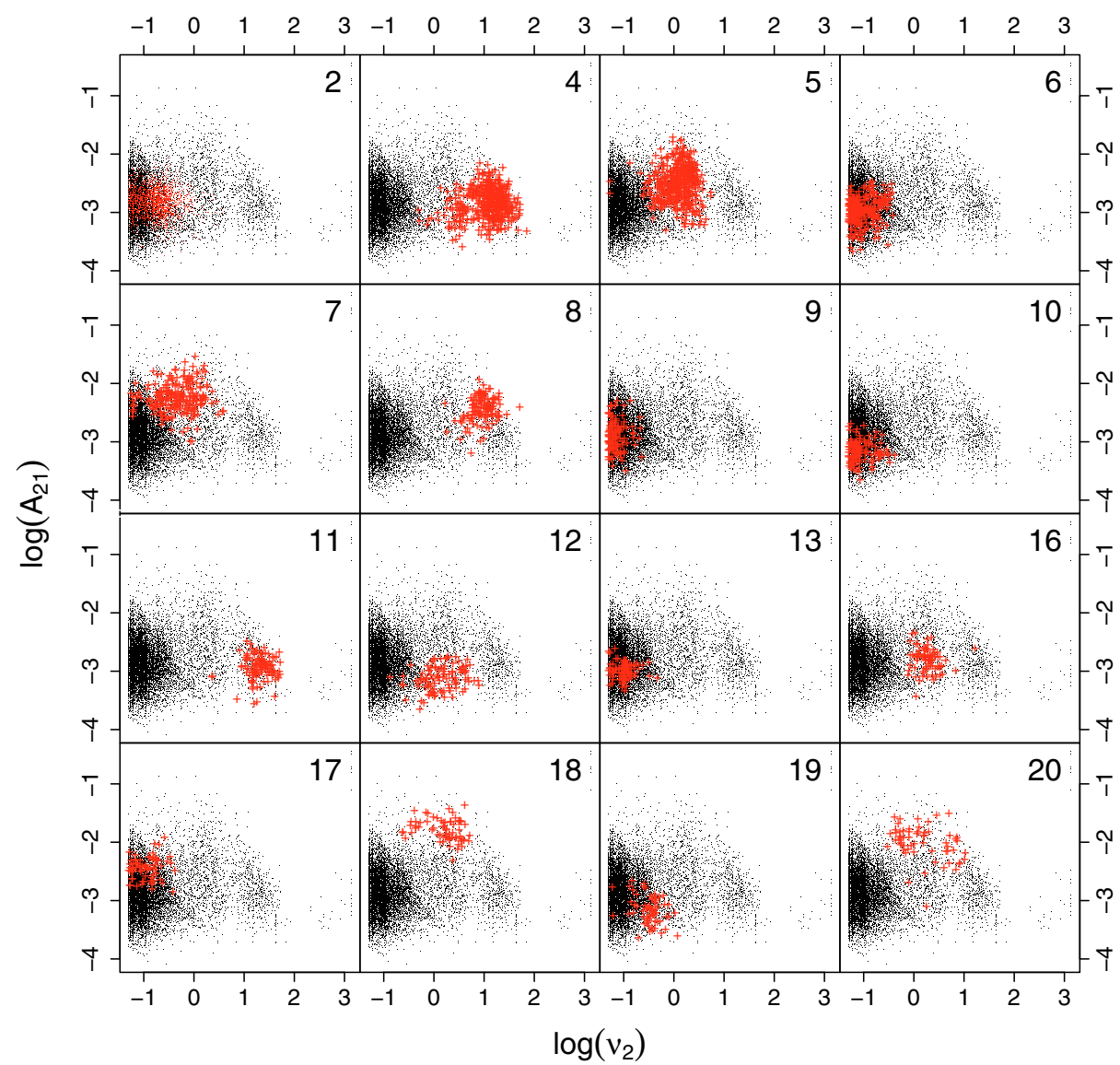

Fig. 45. The clustering structure of the CoRoT first four runs archive at $\sigma=0.2$. The $x$-axis represents the logarithm of the second frequency and the $y$-axis, the logarithm of the amplitude of the first Fourier component of the second detected frequency. Black dots represent the complete database and red crosses identify cluster members. 


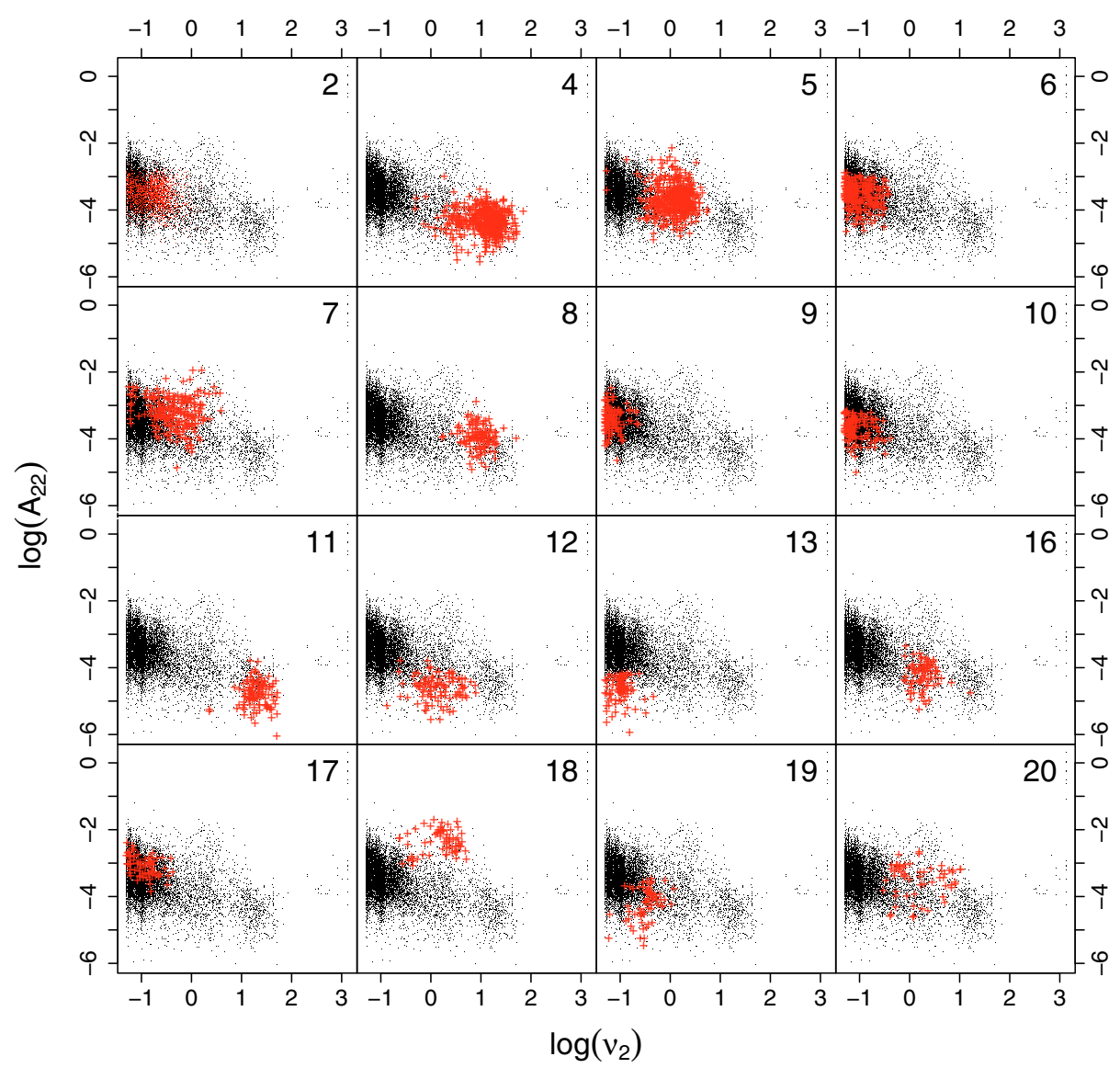

Fig. 46. The clustering structure of the CoRoT first four runs archive at $\sigma=0.2$. The $x$-axis represents the logarithm of the second frequency and the $y$-axis, the logarithm of the amplitude of the second Fourier component of the second detected frequency. Black dots represent the complete database and red crosses identify cluster members.

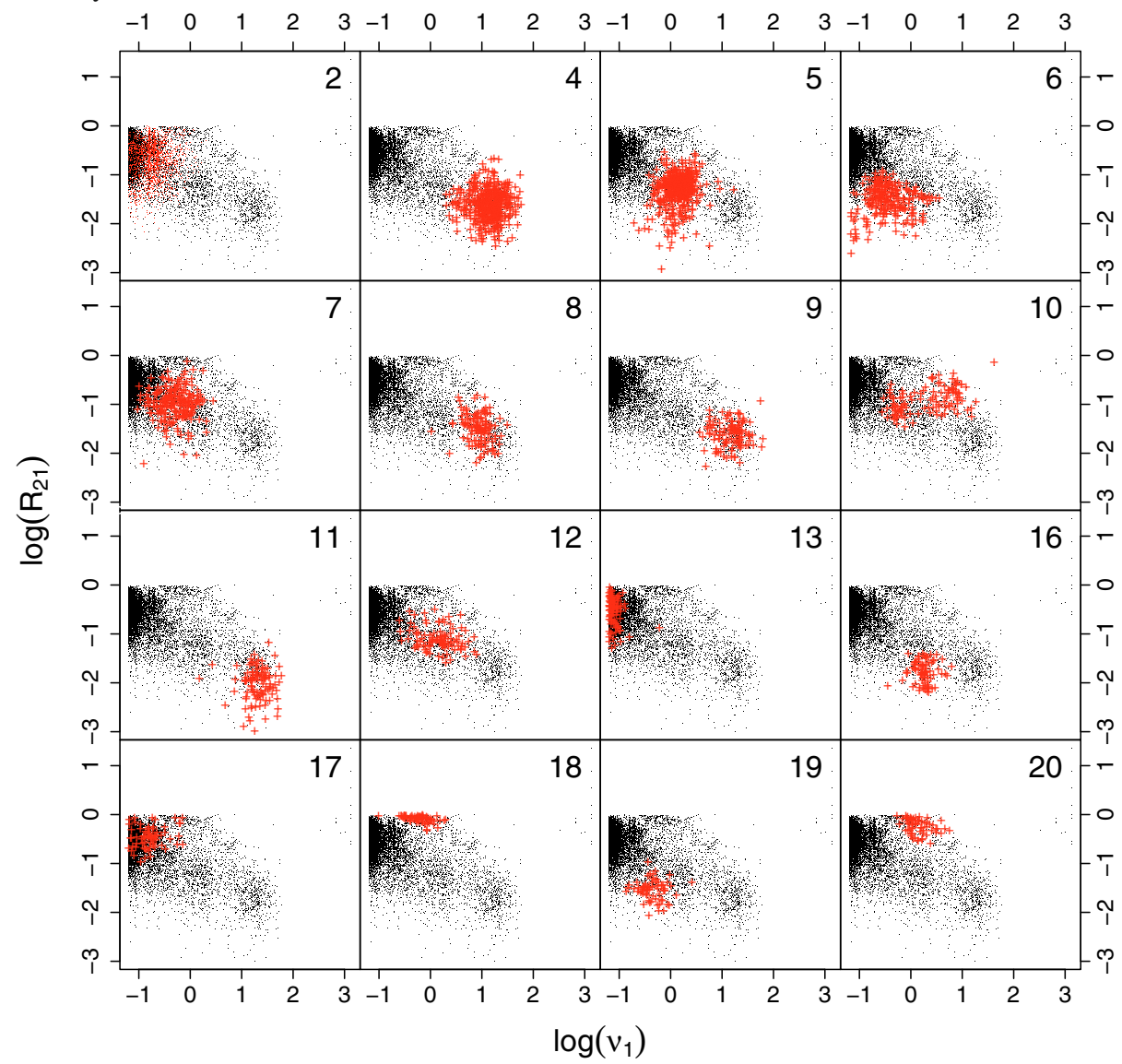

Fig. 47. The clustering structure of the CoRoT first four runs archive at $\sigma=0.2$. The $x$-axis represents the logarithm of the first frequency and the $y$-axis, the logarithm of the $R_{21}$ ratio between the amplitudes of the first two components of the first frequency in the Fourier decomposition. Black dots represent the complete database and red crosses identify cluster members. 


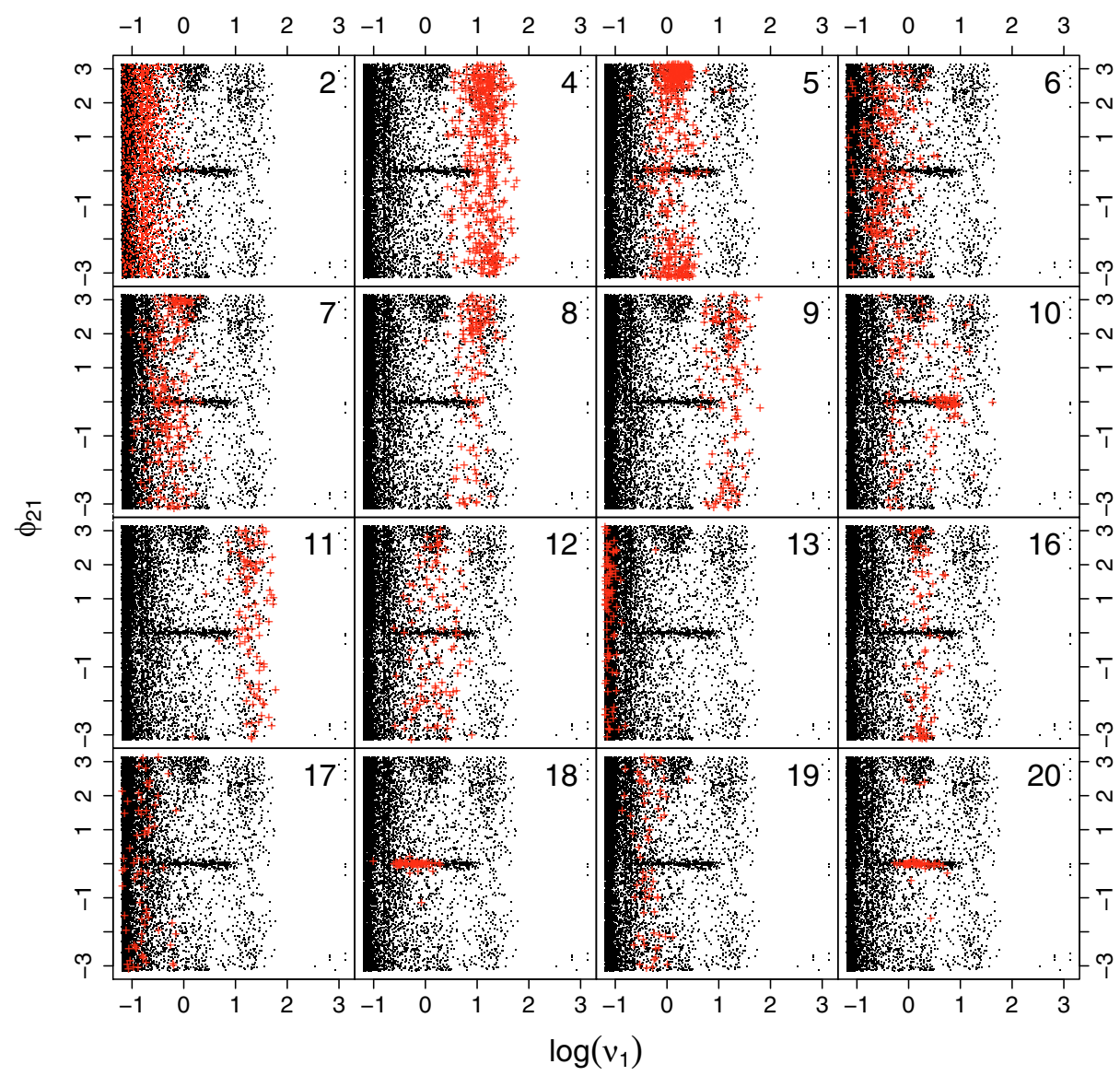

Fig. 48. The clustering structure of the CoRoT first four runs archive at $\sigma=0.2$. The $x$-axis represents the logarithm of the first frequency and the $y$-axis, the phase difference $\phi_{12}$ between the first two components in the Fourier decomposition of the first frequency. The $\phi_{12}$ attribute was not used for clustering and it is only used here for illustration purposes. Black dots represent the complete database and red crosses identify cluster members.

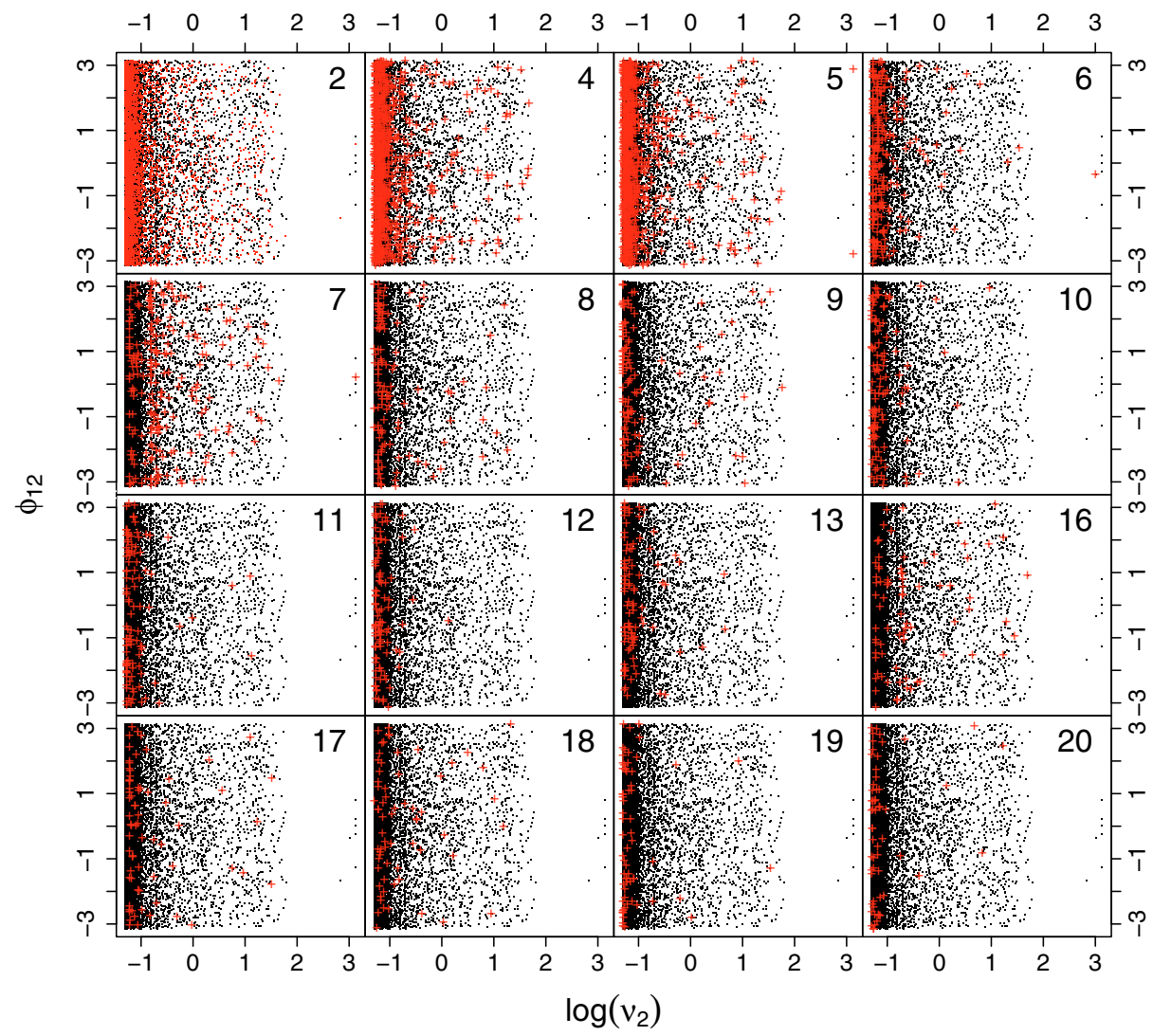

Fig. 49. The clustering structure of the CoRoT first four runs archive at $\sigma=0.2$. The $x$-axis represents the logarithm of the second frequency and the $y$-axis, the phase difference $\phi_{12}$ between the first two components in the Fourier decomposition of this second frequency. The $\phi_{12}$ attribute was not used for clustering and it is only used here for illustration purposes. Black dots represent the complete database and red crosses identify cluster members. 


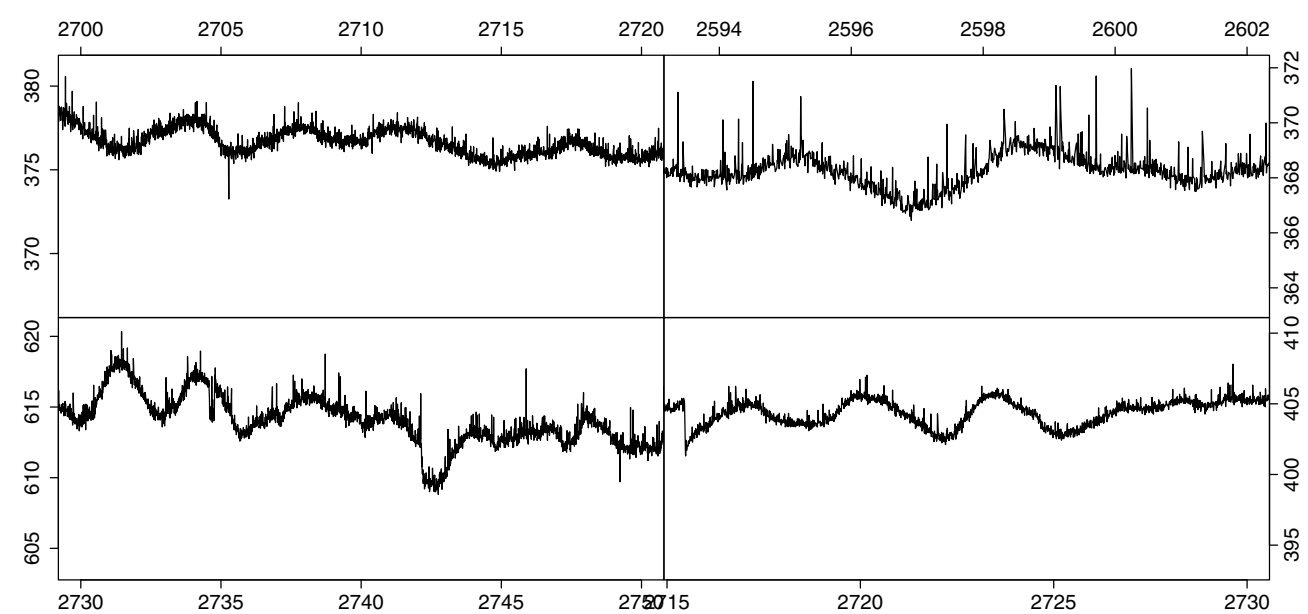

Fig. 50. Close-up of the CoRoT photometric time series of the objects closest to the cluster 6 mode. The $x$ axis represents the modified heliocentric Julian Date, and the $y$ axis, the signal measured in units of $10^{3}$ counts.

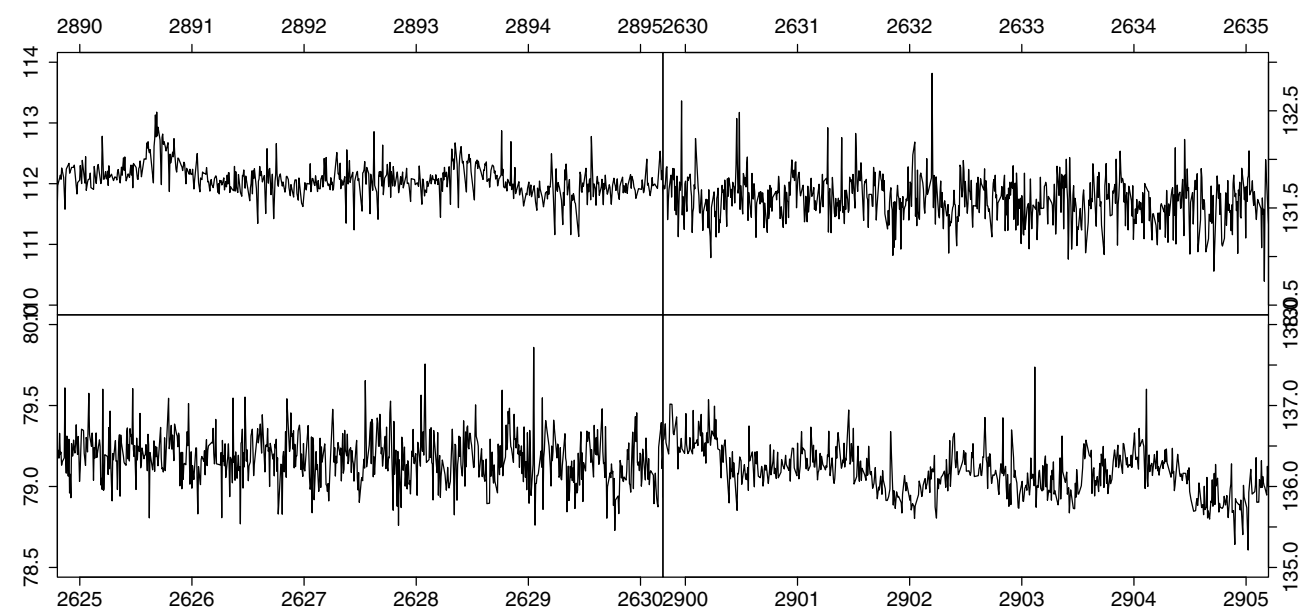

Fig. 51. Close-up of the CoRoT photometric time series of the objects closest to the cluster 10 mode. The $x$ axis represents the modified heliocentric Julian Date, and the $y$ axis, the signal measured in units of $10^{3}$ counts.

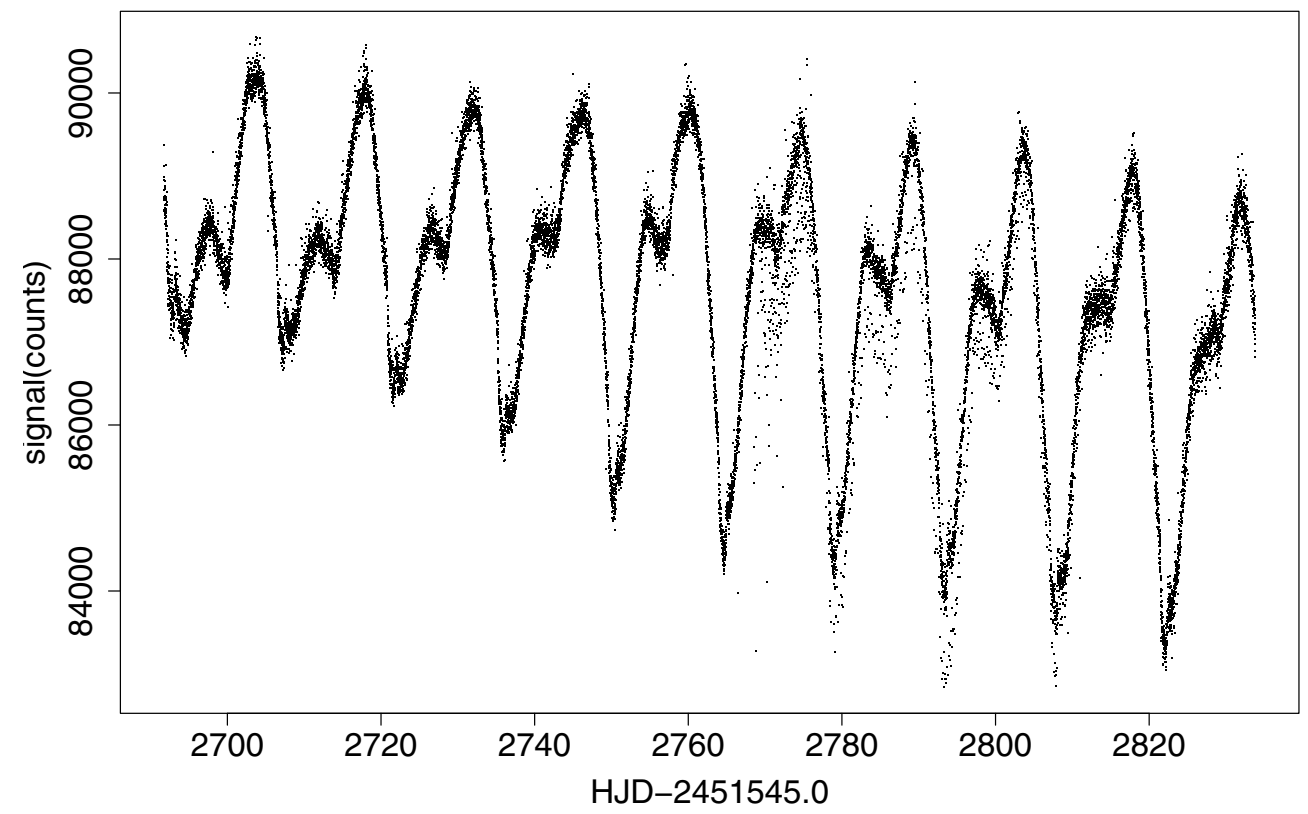

Fig. 52. Close-up of the CoRoT photometric time series of the object closest to the cluster 17 mode. The $x$ axis represents the modified heliocentric Julian Date, and the $y$ axis, the signal measured in counts. 


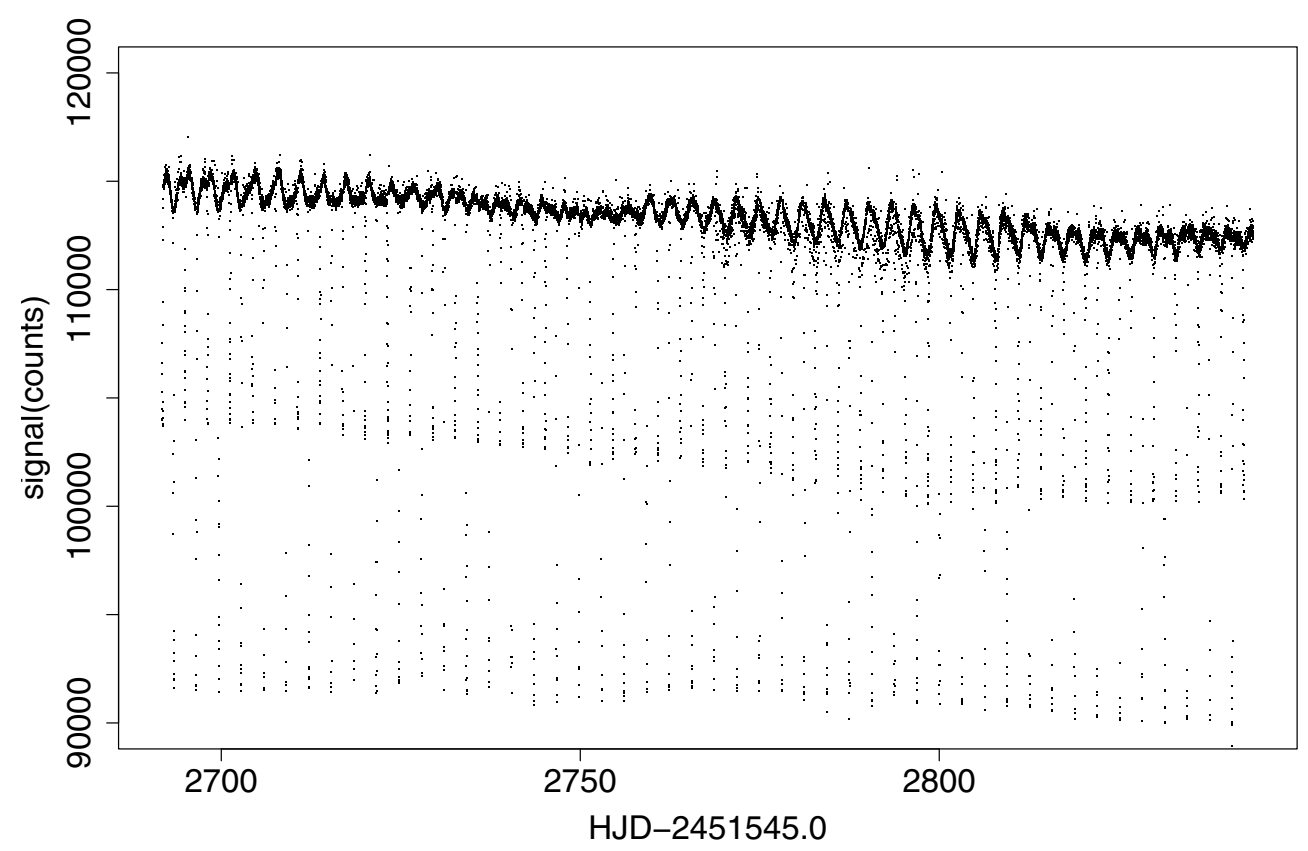

Fig. 53. Close-up of the CoRoT photometric time series of the object closest to the cluster 18 mode (eclipsing binaries). The $x$ axis represents the modified heliocentric Julian Date, and the $y$ axis, the signal measured in counts. 